Board of Governors of the Federal Reserve System

International Finance Discussion Papers

Number 973

May 2009

\title{
The Impact of Macroeconomic Announcements on Real Time Foreign Exchange Rates in Emerging Markets
}

\author{
Fang Cai
}

Hyunsoo Joo

Zhiwei Zhang

NOTE: International Finance Discussion Papers are preliminary materials circulated to stimulate discussion and critical comment. References to International Finance Discussion Papers (other than an acknowledgment that the writer has had access to unpublished material) should be cleared with the author or authors. Recent IFDPs are available on the Web at www.federalreserve.gov/pubs/ifdp/. This paper can be downloaded without charge from Social Science Research Network electronic library at http://www.ssrn.com/. 


\title{
The Impact of Macroeconomic Announcements on Real Time Foreign Exchange Rate in Emerging Markets
}

\author{
Fang Cai, Hyunsoo Joo, Zhiwei Zhang*
}

\begin{abstract}
This paper utilizes a unique high-frequency database to measure how exchange rates in nine emerging markets react to macroeconomic news in the U.S. and domestic economies from 2000 to 2006. We find that major U.S. macroeconomic news have a strong impact on the returns and volatilities of emerging market exchange rates, but many domestic news do not. Emerging market currencies have become more sensitive to U.S. news in recent years. We also find that market sentiment could sway the impact of news on these currencies systematically, as good (bad) news seems to matter more when optimism (pessimism) prevails. Market uncertainty also interacts with macroeconomic news in a statistically significant way, but its role varies across currencies and news.
\end{abstract}

Key words: exchange rate, emerging market, macroeconomic news, high-frequency data

JEL Classification: F31, G14, G15

\footnotetext{
${ }^{*}$ Cai is at the Division of International Finance of the Federal Reserve Board, Washington, DC, 20551, and can be reached at fang.cai@frb.gov or (202) 452-3540. Joo is at the University of Maryland, and can be reached at joo@econ.bsos.umd.edu. Zhang is at the Hong Kong Monetary Authority, and can be reached at zzhang@hkma.gov.hk. We are grateful to David Bowman, Alain Chaboud, Clara Vega, Jon Wongswan and participants in the IF workshop and HKMA workshop for helpful discussions. The views in this paper are solely the responsibility of the author and should not be interpreted as reflecting the views of the Board of Governors of the Federal Reserve System or any other person associated with the Federal Reserve System.
} 


\section{Introduction}

Information transmission across foreign exchange markets has become a widely studied topic in the academic literature. One strand of this literature focuses on the impact of macroeconomic data announcements on foreign exchange markets. Andersen et al. (2003) (ABDV (2003) hereafter) finds that news about macroeconomic fundamentals affect both conditional mean returns and volatilities of exchange rates for major currencies. Some other recent papers in this vein include Andersen et al. (2007), Dominguez and Panthaki (2006), Ehrmann and Fratzscher (2005), Fair (2003), Chaboud et al. (2004), Laakkonen (2004), and Faust et al. (2007). Evans and Lyons (2008) connect the impact of news in the FX market to order flows. Most existing studies have however been limited to major currencies exchange rates. The price discovery process and the information transmission mechanism in emerging economy foreign exchange markets have not yet been well understood.

This paper is the first to focus on how U.S. and domestic macroeconomic announcements affect exchange rates in nine emerging markets: Czech Republic, Hungary, Indonesia, Korea, Mexico, Poland, South Africa, Thailand, and Turkey. ${ }^{1}$ We construct a unique database that covers high frequency exchange rates for the nine emerging market economies from January 2, 2000 to December 31, 2006. The database is complemented by information from Consensus Forecasts on market expectations for these exchange rates, and data from Bloomberg on market expectations for macroeconomic news and the actual announcement. Although similar databases have been studied for major currencies, this is the first time such data for emerging markets are utilized for economic research.

\footnotetext{
${ }^{1}$ Galati (2000) examines the relationship between trading volumes, volatility and bid-ask spreads in foreign exchange markets in 7 emerging economies, but does not measure the impact of news.
} 
We try to address the following questions in this paper: (i) do macro news announcements move emerging market exchange rates? (ii) have emerging market currencies become more sensitive to news as government controls of foreign exchange (FX) markets have reportedly weakened in some of these countries? (iii) how does "market sentiment" affect the way emerging market currencies respond to news? and (iv) does uncertainty in the FX market affect how these currencies react to news?

We find that the answer to the first question depends on whether the news is about the U.S. or the emerging economies and varies across countries. Domestic macro news in emerging markets generally do not have significant effect on exchange rates, with the notable exception for Czech Republic. The set of U.S. macro news that moves major currencies significantly turns out to affect 6 out of 9 emerging market currencies in the same direction in the sample. For the other three currencies, Mexican Peso also reacts to U.S. news significantly but almost always in the opposite direction, while the Thai baht and the Turkish lira rarely respond to U.S. news significantly.

We find evidence that exchange rates in emerging markets have become more sensitive to U.S. news in recent years, probably due to loosened government controls of the FX markets in some of these countries. This pattern is clear for most exchange rates in the sample except for Thailand, where the Thai baht's lack of reaction to news is persistent throughout the sample. The other two Asian currencies, the Korean won and the Indonesian rupiah, used to be nonresponsive foreign and domestic macro news in the early part of the sample, but became more influenced by U.S. news in recent years.

Do macro news have more effect on emerging market currencies when "market sentiment" is strong, i.e., investors expect the currencies to move substantially in one 
direction? The answer is yes. We find strong evidence across country and macro news that market reaction is reinforced by investors' conviction on the direction of the emerging market currencies. The magnitude of this reinforcement effect is large. For instance, when investors expect the Indonesian rupiah to appreciate by 5 percent, the effect of news on nonfarm payroll in the U.S. on the Indonesian rupiah became twice as much as when investors expect the Korean won to stay unchanged.

Does market uncertainty amplify or dampen the impact of news on exchange rates? The answer is ambiguous. While regressions show that market uncertainty dampens more news than it amplifies, the evidence is not overwhelmingly one-sided. In some special cases, the effect of uncertainty on the same news differs across countries. Further analysis on this issue is necessary.

Our paper complements other studies on the impact of news on asset prices in emerging markets. Wongswan (2006) provides evidence of transmission of information from the U.S. and Japan to Korean and Thai equity markets. Using high-frequency intraday data, he finds a large and significant association between emerging-economy equity volatility and trading volume and developed-economy macroeconomic announcements at short time horizons. Andritzky, Bannister, and Tamirisa (2007) examine how emerging market bonds react to macroeconomic announcements and find that global bond spreads respond to rating actions and changes in U.S. interest rates rather than domestic data and policy announcements. Consistent with their studies, we find a significant impact of major U.S. macroeconomic news on emerging market currencies using high frequency data. Compared with their papers, the innovations of our work are: (a) the longer sample of our data makes it 
possible to track the evolution of reactions to news in the emerging currency markets, and (b) the reaction of exchange rates to news is linked to market sentiment and uncertainty.

The rest of the paper proceeds as follows. Section II provides the description of the data. Section III presents the econometric specifications and the estimates of how news surprises affect exchange rate returns and volatility in the nine emerging markets. Section IV shows how market forecasts and uncertainty interact with macro news and affect exchange rates in emerging markets. Section V concludes.

\section{Data}

\section{Exchange Rate Data}

The paper uses high-frequency exchange rate data for nine emerging markets, drawn from Olsen Financial Technologies. The data report exchange rates of the nine EM currencies versus U.S. dollar at 5-minute intervals. The full sample period is from January 2, 2000 to December 31, 2006, covering 2,557 days of bid-ask prices for each currency with two exceptions ${ }^{2}$. It should be noted that the dataset has quite many missing values, in particular for the earlier years. The number of non-missing values for bid and ask price of each country's exchange rate is presented in Appendix $1 .^{3}$

Using bid-ask price quotes from the raw exchange rate data, we take the simple arithmetic average to get the middle price quote. Then we calculate 5-minute currency

\footnotetext{
${ }^{2}$ For KRW/USD, January 2004 (31 days) data are not included. For TRY/USD, the sample period is from January 2, 2001 to December 31, 2006. The number of observations of high frequency FX data is 2,557*288 $=736,416$ for Czech Republic, Hungary, Indonesia, Mexico, Poland, South Africa, and Thailand, 727,488 for Korea, and 631,008 for Turkey.

${ }^{3}$ We also estimate the same model with fully filled data using interpolation for missing values. The estimates with interpolated data show similar but a little bit weaker results compared with the results presented in this paper.
} 
returns by taking log differences. We multiply the log differences of currency returns by 100 to obtain log currency returns. Following ABDV (2003), we exclude data on weekends and national holidays, since the quoted prices may have some bias based on low transaction volumes. First, we drop the period from Friday 21:05 to Sunday 21:00 (local time) for weekends. Second, we drop national holidays in the U.S. and the nine emerging markets. In order to check how the series of currency returns vary over time, we plot autocorrelations of the currency returns and its absolute value in Figures 1 and 2, respectively. The general pattern of the two figures is similar with what previous studies show for major currencies: the autocorrelations of currency returns are statistically significant in the short term, and decay fast; the autocorrelations of absolute returns are statistically significant in the short term and stay high persistently.

In addition, it should be taken into account that the exchange rate regimes in some emerging markets (such as Hungary, Poland, and Turkey), might have changed within the sample period (Appendix 2). The Hungarian foreign exchange regime changed from a crawling peg to a pegged exchange rate within horizontal band in October 2001, and devalued on June 2003. For the Polish zloty, a crawling peg based on 55\% of Euro and 45\% of dollar changed into independent floating on April 2000. For the Turkish lira, many changes happened during the sample period due to the financial crisis in 2001. The regime changed from a crawling peg to independent floating on February 2001, and the New Turkish lira was introduced on 2005 and became a sole legal tender from January 1, 2006 with a conversion rate of YTL $1=$ TL 1 million. We convert all previous TL quotes into YTL based on the conversion rate when calculating log returns of its exchange rate 


\section{Actual and Predicted Economic Variables}

We use economic forecast data from Bloomberg on various actual and predicted economic indices in the U.S. and nine emerging market countries. ${ }^{4}$ Many economists and analysts in the financial markets who use Bloomberg submit their own forecasts to Bloomberg. However since such forecast data submission is voluntary, the number of the observations varies for each observation of economic index. For instance, 39 people submitted forecasts of the initial jobless claims in the U.S. that is published by the Department of Labor on January 5, 2008. Based on those forecasts, Bloomberg provides mean, median, maximum, and minimum values for each economic index. In some cases (mostly in emerging markets) the forecasts are based on the views from a small number of economists. We drop all forecasts that are based on views from fewer than 5 economists.

In Table 1, we present the number of the observations for each variable used in the empirical analysis. There are 26 indices for the U.S. news, 13 for Czech Republic, 11 for Mexico and Poland, 9 for Turkey, 8 for South Africa, 7 for Hungary, 6 for Korea, 5 for Thailand, and 4 for Indonesia. Since the unit of each economic index is different, we standardize the time series of each economic index by calculating the surprise as (actual number - forecasts) divided by its sample standard deviation

$$
S_{k t}=\frac{A_{k t}-F_{k t}}{\hat{\sigma}_{k}}
$$

\footnotetext{
${ }^{4}$ These forecasts of economic indices are obtained from ECO menu in the Bloomberg terminal by the country.
} 
where $A_{k t}$ is the actual announced value for economic index $k$ at time $t, F_{k t}$ is the mean of forecasts, and $\hat{\sigma}_{k}$ is sample standard deviation of $A_{k t}-F_{k t}$.

\section{Foreign Exchange Forecasts}

To measure market expectations on exchange rates, we use forecast data from Consensus Forecasts, which provides a simple arithmetic average of the forecasts for foreign exchange rates over 90 countries as well as major economic indices on a monthly or bimonthly basis. ${ }^{5}$ The exact date when the survey is conducted is shown in the published data. We collect information on the survey date, spot rate on the survey date, sample average, maximum, minimum, and standard deviation of each exchange rate for the following 1, 3, 12 and 24 months. A variable is constructed to measure which direction the market anticipates the exchange rates move,

$$
F X D_{i, d, t}=\frac{C F X_{i, d, t}-S F X_{i, t}}{S F X_{i, t}}
$$

where $C F X_{i, d, t}$ is consensus forecast for country $i$ 's exchange rate at day $t$ for the next $d$ months, and $S F X_{i, t}$ is the spot exchange rate on day $t$. If $F X D_{i, d, t}$ is positive, then market participants expect that local currency $i$ will depreciate for next $d$ months, and vice versa. In Appendix 3a, we provide summary statistics for $F X D$.

\footnotetext{
${ }^{5}$ Monthly forecasts for Asian economies are available for the full sample. For Latin American economies, monthly forecasts are available after May 2001, and bimonthly forecasts are available before. For other economies, only bimonthly forecasts are available.
} 


\section{Announcements and FX responses}

\section{Contemporaneous Effect from OLS Regressions}

We start by running an OLS regression

$$
R_{i, t}=\beta_{i, k} S_{i, k, t}+\varepsilon_{i, t}
$$

where $\mathrm{R}_{i, t}$ denotes 5-minute exchange rate returns from time $t$ to time $t+1$ in country $i, \mathrm{~S}_{i, k, t}$ is the surprise of macroeconomic news $k$ at time $t$ in country $i$. The estimates are based on only those observations $\left(\mathrm{R}_{i, t}, \mathrm{~S}_{i, k, t}\right)$ such that an announcement was made at time $t$. This specification has the advantage of simplicity. The drawback is that it does not control for the potential dynamic feature of exchange rates and news, and does not correct for heteroskedastic disturbances in the error terms. We will move to a more sophisticated model in the next subsection that addresses these issues.

Table 2 shows the estimates from these regressions. For comparison, we also examine the impact of U.S. news on the euro/dollar exchange rate. Three features stand out. First, exchange rates for South Africa and emerging markets in Europe react to many U.S. news in a similar way as major currencies do (as documented in previous literature), but many of the same news have little effect on currencies in Asia and Turkey. Second, most domestic macroeconomic news have no impact on EM exchange rates. Finally, the euro exchange rate responds to major U.S. news in a similar way to European emerging market currencies. We elaborate more on these findings before moving to the more sophisticated specification. 
In the case of U.S. news, positive surprises on consumer confidence, durable goods order, GDP, non-farm payroll, retail sales and trade balance lead to depreciation of EM currencies in Czech Republic, Hungary, Poland, and South Africa, with a few exceptions. This set of news is also found to be significant in ABDV (2003). New home sales turns out to be highly significant for emerging markets in Europe, reflecting the importance of the U.S. housing sector for the sample we study. On the other hand, very few U.S. news have significant impact on the Mexican, Turkish and Asian currencies. Of the 26 U.S. news we studied, only 3 show up significantly for Korea, 6 for Indonesia, 1 for Thailand, 3 for Mexico, and 2 for Turkey.

In contrast with the large number of significant U.S. news, few domestic news in emerging markets have a significant impact on their exchange rates. For Indonesia, Thailand, and Turkey, no domestic news are significant in the regressions. Even for Hungary and Poland where many U.S. news move exchange rates significantly, only one domestic news is significant in each country. Of the 14 cases where domestic news announcements move the exchange rates, 9 cases are related to domestic growth or external balance: the current account in Czech Republic and Poland, GDP in Czech Republic and Mexico, industrial production in Hungary, and the trade balance in Czech Republic, Mexico, and South Africa.

Given the long sample of the dataset, we can examine if exchange rates in emerging markets have become more sensitive to news in recent years. We estimate equation (3) using a two-year rolling window, and plot the point estimates of $\beta_{i, k}$ over time. Charts in Figure 3 plot the significant estimates from such rolling regressions. Two patterns stand out. First, most EM currencies have become more sensitive to news in recent years than before. For instance, in Korea, few U.S. news had significant impact on the won before late 2002, while 
4 out of 9 news are persistently significant in recent years. Thailand is an exception, where Thai baht barely reacts to any U.S. news throughout the whole sample.

Second, the fact that some news do not affect certain currencies cannot be explained by the lack of observations. In the later part of the sample, the numbers of observations for given U.S. news are fairly equal across countries. Yet, some currencies persistently react to news, while others seem to be irresponsive.

\section{Contemporaneous Effect from Dynamic Regressions with Heteroskedesticity}

We follow ABDV (2003) in their econometric specifications to include lag terms of currency returns and news, and control for heteroskedestic errors. First, we estimate a linear regression model based on $I$ lags of 5-minute returns, and $J$ lags for all the news surprises.

We choose the lags $I=5$ and $J=2$ according to the Akaike Information Criteria and

Schwartz Criteria ${ }^{6}$ The number of news surprises in the model is different for each country since that of the domestic news surprises is different.

$$
\begin{aligned}
R_{t} & =\beta_{0}+\sum_{i=1}^{I} \beta_{i} R_{t-i}+\sum_{k=1}^{K} \sum_{j=0}^{J} \beta_{k j} S_{k, t-j}+\varepsilon_{t} \\
t & =1, \ldots, T . \\
\left|\hat{\varepsilon}_{t}\right| & =c+\psi \frac{\hat{\sigma}_{d(t)}}{\sqrt{288}}+\sum_{k=1}^{K} \sum_{j^{\prime}=0}^{J^{\prime}} \beta_{k j^{\prime}}\left|S_{k, t-j^{\prime}}\right|+\left(\sum_{q=1}^{Q}\left(\delta_{q} \cos \left(\frac{q 2 \pi t}{288}\right)+\phi_{q} \sin \left(\frac{q 2 \pi t}{288}\right)\right)\right)+u_{t}
\end{aligned}
$$

\footnotetext{
${ }^{6}$ The exact AIC and BIC show different optimal numbers of lags across countries. However, 6 of the sample countries show that 5 lags of FX returns are good enough, whereas the other countries show relatively small lags for FX returns.
} 
As in ABDV (2003), the absolute value of the residual from equation 1.4 is modeled as the sum of three terms: daily volatility forecast to measure average volatility level during the day; the absolute value of news surprise including lags to assess the impact from the news; and the Fourier flexible form with trigonometric terms for the calendar effect. Equations 1.4 and 1.5 are estimated by 2 -stage WLS. First, we run an OLS regression with equation (4). Then we estimate equation (5) to get a linear prediction of the absolute value of the residuals in equation (4). Finally, using the linear prediction from equation (5) as a weight, we perform a weighted least-squares estimation of equation (4).

It is necessary to be more specific on the independent variables used in equation (5). The daily level of volatility in the second term is based on the residual from the regression of GARCH $(1,1)$ model using daily spot exchange rate returns from January 1, 1993 as described above in the data description. GARCH $(1,1)$ models are generally used to extract predictions in high-frequency financial data in a wide variety of papers.

The third term represents the impact of news surprise on the volatility. In order to enhance tractability, we impose a polynomial specification on the response patterns associated with $\beta_{k^{\prime}}$, as in ABDV (2003). This ensures that the response patterns related to the news surprise are determined by the restriction we provide on the specifications. Consider the general form of polynomials, $p(\tau)=c_{0}+c_{1} \tau+\ldots+c_{p} \tau^{p}$, for $\tau=0,1, \ldots, J^{\prime}$. The restrictions we apply to this equation are $J^{\prime}=12, p=3$, and $p\left(J^{\prime}\right)=0$. As a result, we have $p(\tau)=c_{0}\left[1-(\tau / 12)^{3}\right]+c_{1} \tau\left[1-(\tau / 12)^{2}\right]+c_{2} \tau^{2}[1-(\tau / 12)]$. Using this equation, we estimate three coefficients for each FX returns and each news surprise, and plug the fixed value from the estimation into the disturbance equation (4). 


$$
\begin{aligned}
\left|\hat{\varepsilon}_{t}\right|=c+ & \psi \frac{\hat{\sigma}_{d(t)}}{\sqrt{288}}+\sum_{k=1}^{K} \sum_{j^{\prime}=0}^{J^{\prime}} \eta_{k}\left\{\hat{c}_{0}\left(1-\left(\frac{j^{\prime}}{12}\right)^{3}\right)+\hat{c}_{1} j^{\prime}\left(1-\left(\frac{j^{\prime}}{12}\right)^{2}\right)+\hat{c}_{2}\left(j^{\prime}\right)^{2}\left(1-\left(\frac{j^{\prime}}{12}\right)\right)\right\}\left|S_{k, t-j^{\prime}}\right| \\
& +\left(\sum_{q=1}^{Q}\left(\delta_{q} \cos \left(\frac{q 2 \pi t}{288}\right)+\phi_{q} \sin \left(\frac{q 2 \pi t}{288}\right)\right)\right)+u_{t}
\end{aligned}
$$

The fourth term of Fourier series covers calendar effects in the model. AIC and Schwartz criteria suggest that $Q=4$ is appropriate for the model, and it means that the seasonal pattern of intra-day trading quote is relatively smooth.

Table 3 presents the estimates for a selected group of U.S. news. Compared with Table 2, emerging market exchange rates react to U.S. news more consistently across countries. Currencies in Thailand and Turkey remain rather insensitive to most U.S. news. For the other 7 countries, all of the 9 major U.S. news have significant signs in the expected direction, with few exceptions. As in the OLS regressions, the Mexican peso's reaction to U.S. news remains mostly the opposite of those of other currencies.

The dynamic structure of this model allows us to estimate the persistence of news effects on exchange rates. The lagged variables of U.S. news surprises mostly show the same sign as the contemporaneous variables. There are some exceptions for news such as Nonfarm Payroll and Producer Prices, which show mean reversion effects across the time. However, the size of impact seems to decay as time goes by.

A complete table with all U.S. and domestic news is provided in Appendix 4. Among domestic news surprises, the consumer price index and current account balance show significance for the contemporary FX impact across the countries. The trade balance and producer price also seem to be significant when lagged variables are considered. Major domestic macroeconomic news surprises in Eastern European countries also have a 
significant impact on their exchange rate returns. For the Czech Republic, the budget deficit, current account, consumer price index, exports, imports, industrial production index, producer price index, retail sales index, and trade balance are all significant in the model. The current account, consumer price index, and industrial production show significance in Hungary. And in Poland, the significant news surprises include current account, GDP, money supply, unemployment, and wholesale sales index. Along with European countries, exchange rate returns in South Africa are strongly responsive to domestic news surprises. Among the 6 domestic macroeconomic announcements we collect, the consumer price index, interest rate, money supply, and trade balance are all statistically significant. In Asian countries, nevertheless, the impact of domestic news surprises on exchange rate returns are somewhat smaller compared with that of the U.S. news surprises. Only one of the domestic news surprises in Thailand is significant in the estimation model. None of the domestic news is significant in Indonesia and Korea.

\section{Announcements and FX Volatility}

In order to assess how the news surprises affect FX volatility, we compare contemporaneous coefficients with the sum of those across 12 lags (i.e., 60 minutes of time) used in the regression model suggested in equation (5). In this case, we concentrate on the 9 news surprises that are statistically significant for at least 6 countries in the current terms or more than 13 including additional 2 lags in equation (4). 
It should be noted that we use equation (5) for the estimation, so the impact of the news surprise on the volatility should last until the next 60 minutes. $^{7}$ Results presented in the middle section of Table 3 suggest that several of the coefficients for news surprises in the volatility equation have statistical significance, although they tend to be smaller compared with the contemporaneous return response coefficients in the top panel. Only 7 of the coefficients for 7 countries excluding Thailand and Turkey are insignificant. Comparing the significance of coefficients in the conditional mean equation (4) with those of volatility equation (5), it can be seen that the news surprises provide more impact on volatility than on conditional mean of exchange rate. To summarize, $87.5 \%$ of 9 major economic news surprises in 9 countries which are statistically significant have a more prolonged impact on volatility for 60 minutes. The whole set of coefficients including contemporaneous and cumulated coefficients are presented in Appendix 4.

As shown in the bottom panel of Table 3, the cumulative response of volatility is much larger than the contemporaneous volatility response, which is consistent with ABDV (2003)'s finding that volatility adjusts to news surprises gradually. An alternative possibility is that the announcement itself can influence on FX market rather than the size of the news surprise. To check for this possibility, we include dummy variables that represent the announcement in both equation (4) and (5) such that the lags should be the same as news surprise. Then the equation model changes as follows:

\footnotetext{
${ }^{7}$ We can extend the time period for this estimation by assigning a bigger number for the time lag J' than 12 , however this may introduce other sources of volatility within the period.
} 


$$
\begin{gathered}
R_{t}=\beta_{0}+\sum_{i=1}^{I} \beta_{i} R_{t-i}+\sum_{k=1}^{K} \sum_{j=0}^{J} \beta_{k j} S_{k, t-j}+\sum_{k=1}^{K} \sum_{j=0}^{J} \theta_{k j} D_{k, t-j}+\varepsilon_{t} \\
t=1, \ldots, T \\
\left|\hat{\varepsilon}_{t}\right|=c+\psi \frac{\hat{\sigma}_{d(t)}}{\sqrt{288}}+\sum_{k=1}^{K} \sum_{j^{\prime}=0}^{J^{\prime}} \beta_{k j^{\prime}}\left|S_{k, t-j^{\prime}}\right|+\sum_{k=1}^{K} \sum_{j^{\prime}=0}^{J^{\prime}} \theta_{k j^{\prime}} D_{k, t-j^{\prime}}+\left(\sum_{q=1}^{Q}\left(\delta_{q} \cos \left(\frac{q 2 \pi t}{288}\right)+\varphi_{q} \sin \left(\frac{q 2 \pi t}{288}\right)\right)+u_{t}\right.
\end{gathered}
$$

As before, we present major 9 economic indices that show significant impact on FX markets across the countries in Table 4. The set of all coefficients can also be found in Appendix 5. In Table 4, many major economic indicators seem to have an announcement effect on FX changes even after taking into account the news surprise impact. Furthermore, the announcement effects exist not only for FX returns but also for the volatility.

\section{Testing for Asymmetry}

We test if there is any asymmetry in the impact of the news surprises according to the sign. ABDV (2003) reports asymmetric response of US news in the case of major currencies. The long sample and the large number of currencies in our sample provide a good opportunity to check if such patterns also exist in emerging markets. First, we divide news surprises into two groups based on their signs, and estimating two equations below:

$$
R_{t}= \begin{cases}\beta_{0 k} S_{k t}+\beta_{1 k} S_{k t}^{2}+\varepsilon_{t} & \text { if } S_{t} \leq 0 \\ \beta_{2 k} S_{k t}+\beta_{3 k} S_{k t}^{2}+\varepsilon_{t} & \text { if } S_{t}>0\end{cases}
$$

With this estimation, we reconstruct the set of graphs that contain the fitted value on the vertical axis and the standard deviation of the news surprise in horizontal axis in Figure 4 
(using the average impact over all news surprises). There appear to be some differences between the two subgroups. To investigate this more formally, we try a modified equation to test if there is any asymmetry across the sign of the news surprise

$R_{t}=\beta_{0 k} S_{k t}+\beta_{1 k} S_{k t}^{2}+D_{k t}\left(\beta_{2 k} S_{k t}+\beta_{3 k} S_{k t}^{2}\right)+\varepsilon_{t}$

where $D_{k t}$ denotes a dummy variable which takes the value 1 if the news surprise is positive, and the value of 0 if negative. To test for asymmetry, we define the null hypothesis such that FX returns have symmetry $\left(\beta_{2 k}=0\right.$, and $\left.\beta_{3 k}=0\right)$ for major 9 economic indicators. The results of the test are presented below in Table 5 . Only 9 cases suggest that the symmetry hypothesis is rejected at $5 \%$ significance, while 72 other cases can not reject the symmetric null hypothesis.

This symmetric impact of news surprises on FX returns is in contrast with the findings in ABDV (2003). To look into the source of this difference, we repeat the regression above for euro. As it turns out, the euro responds to most U.S. news in a symmetric way as well in our sample, suggesting that the different findings between ours and ABDV (2003) come from the different sample periods rather than differences between emerging market currencies and major currencies.

\section{Market Sentiment, Uncertainty, and Macroeconomic News}

In this section, we examine the interaction between market sentiment on emerging market currencies and the exchange rate response to news surprises. For instance, if market participants expect that Korean won will depreciate in a near future as a consensus, then the 
news surprise that suggests the U.S. economy be stronger than expected may have a greater impact on returns of Korean won by making this currency depreciating more rapidly, and vice versa. Therefore, this case consists of two different expectational errors from market participants: a first error from news expectations, and a second error consisting of an FX forecast error. On the other hand, if we can think that the expectation of future appreciation or depreciation is related to the economic cycle in a country, then this approach may become the alternative way to assess symmetry in the impact described in the above section. We use the median value of 1-month-ahead FX forecasts from Consensus Forecasts as a proxy of market expectation of each currency.

We use an ordinary least square regression with some modification in equation (4), by adding an FX consensus variable multiplied by news surprises. If the hypothesis described above is true, then the coefficients on the interaction variable will be positive. The modified equation is as follows:

$$
\begin{aligned}
R_{t} & =\beta_{0}+\sum_{i=1}^{I} \beta_{i} R_{t-i}+\sum_{k=1}^{K} \sum_{j=0}^{J} \beta_{k j} S_{k, t-j}+\sum_{k=1}^{K} \sum_{j=0}^{J} \gamma_{k j} F X D_{d, t-j} S_{k, t-j}+\varepsilon_{t} \\
t & =1, \ldots, T .
\end{aligned}
$$

In Table 6, we focus on 9 U.S. major economic indices discussed earlier. All the coefficients for variables used in this regression are presented in Appendix 6. The first part of the table presents coefficients for news surprises only, and the second part for FX forecasts (FXD) multiplied by the news surprises. Notably, many of the FX forecast-related coefficients show statistically significant and positive values, suggesting that market sentiment plays an important role in how news surprises move EM currencies. It acts as an amplifier when the market is pessimistic (optimistic) about the EM currencies and news 
surprises suggest stronger (weaker) U.S. economy. For instance, if market analysts think that the Czech Republic koruna will depreciate (appreciate) by $10 \%$ for next $d$ months and the durable good orders' data is 1 standard deviation higher (lower) than expected, then exchange rate returns will depreciate (appreciate) 2.2 basis points more than when no exchange rate change is expected for next $d$ months. ${ }^{8}$ On the other hand, when the EM currencies are under pressure to appreciate, positive sentiment for these currencies works as a shock absorber against strong U.S. news. This evidence is consistent with the findings of Mian and Sankaraguruswamy (2008) that the stock market response to good (bad) news is greater during a high (low) sentiment period. One explanation for these results is investor overconfidence as documented in Barberis and Thaler (2003) and Hirshleifer (2001), i.e., investor are more likely to accept news that is in line with their prior beliefs and ignore information that is contradictory to their prior beliefs.

The accelerator effect of market sentiment provides a potential explanation why we find no evidence for asymmetry in EM currencies' reaction to news as in ABDV (2003). EM currencies experience more ups-and-downs than major currency pairs. The long sample of our dataset contains both periods of market optimism and pessimism for each EM currency. Over the market sentiment cycle, this asymmetry might be averaged out. In contrast, ABDV (2003)'s sample period covers one side of the business cycle, when market sentiment might be persistently one-sided as well.

We further test the effect of uncertainty on exchange rate response to news. The specification is

\footnotetext{
${ }^{8}$ Since we multiply log difference of FX by 100 to increase the scale of coefficients, we need to divide by 100 again, so that the magnitude of the shock can be measured correctly.
} 


$$
R_{t}=\beta_{0}+\sum_{i=1}^{I} \beta_{i} R_{t-i}+\sum_{k=1}^{K} \sum_{j=0}^{J} \beta_{k j} S_{k, t-j}+\sum_{k=1}^{K} \gamma_{k} D I S P_{d, t} S_{k, t}+\varepsilon_{t}
$$

where DISP is a measure of market uncertainty defined by the dispersion of market forecasts for each EM currency. It is constructed as $D I S P_{d, t}=\left|\frac{C F X_{d, t}^{\text {high }}-C F X_{d, t}^{\text {low }}}{S F X_{t}}\right|$, where $C F X_{d, t}^{\text {high }}$

denotes the maximum of FX forecasts at time $t$, and $C F X_{d, t}^{\text {low }}$ denotes the minimum of FX forecasts at time $t$ (the summary statistics for DISP is presented in Appendix 3b).

The role of market uncertainty in these regressions is not conclusive. The estimates are shown in Table 7. Despite many significant estimates, the signs of the parameters for market uncertainty vary across country and across news. The diverse set of parameters leaves the regressions inconclusive. Nonetheless, the fact that market uncertainty shows significance in many regressions indicates it does have influence on how exchange rates react to news, but the channel of such influence is not yet well understood.

\section{Conclusion}

This paper documents some interesting features in the FX market for emerging market currencies. Except for Thailand and Turkey, whose currencies are not sensitive to news, the other 7 currencies show consistent reactions to news. First, U.S. news matters much more than domestic news. Second, currencies have become more sensitive in recent years than before. Third, market sentiment on these currencies plays an important role by swaying the impact of news surprises, i.e., good (bad) news matters more when optimism (pessimism) prevails. These finding are robust across countries and news we studied. 
The role of uncertainty in FX market is also studied but is not fully explored. The significant yet inconclusive estimates indicate that its role could be state-dependent, and we are not yet able to capture what is the missing state variable. On the role of market sentiment, although we found significant and consistent results for emerging markets, it is not clear if this is a unique phenomenon for emerging market currencies, or it also exists for major currencies and other financial assets. These are potential topics for future research. 


\section{References}

Andersen, Torben G., Tim Bollerslev, Francis X. Diebold, and Clara Vega, 2003, "Micro Effects of Macro Announcements: Real-Time Price Discovery in Foreign Exchange." The American Economic Review, 93(1), pp.38-62.

Andersen, Torben G., Tim Bollerslev, Francis X. Diebold, and Clara Vega, 2007, "RealTime Price Discovery in Global Stock, Bond and Foreign Exchange Markets.” Journal of International Economics, 73(2), pp.251-277.

Andritzky, Jochen R, Geoffrey J. Bannister, and Natalia T. Tamirisa, 2007, "The impact of macroeconomic announcements on emerging market bonds." Emerging Markets Review, $8(1)$, pp.20-37.

Barberis, N. and R. Thaler, 2003, "A Survey of Behavioral Finance," Handbook of the Economics of Finance, volume 1, part 2, pp.1053-1128.

Bollerslev, Tim, Jun Cai, Frank M. Song, 2000, “Intraday Perodicity, Long Memory Volatility, and Macroeconomic Announcement Effects in the US Treasury Bond Market." Journal of Empirical Finance, 7(1), pp.37-55.

Chaboud, A., Chernenko, S., Howorka, E., Iyer, R., Liu, D., Wright, J., 2004, “The highfrequency Effects of U.S. Macroeconomic Data Releases on Prices and Trading Activity in the Global Interdealer Foreign Exchange Market." Board of Governors of the Federal Reserve System, IFDP No. 823.

Cheung, Yin-Wong, Menzie D. Chinn, Ian W. Marsh, 2004, "How Do UK-based Foreign Exchange Dealers Think Their Market Operates?" International Journal of Finance and Economics, 9(4), pp.289-306.

Dominguez, Kathryn M.E., Freyan Panthaki, 2006, "What defines 'News' in Foreign Exchange Markets?” Journal of International Money and Finance, 25(1), pp. 168-198.

Ederington, Louis H., Jae Ha Lee, 1996, "The Creation and Resolution of Market Uncertainty: The Impact of Information Releases on Implied Volatility." The Journal of Financial and Quantitative Analysis, 31(4), pp.513-539.

Ehrmann, Michael, Marcel Fratzscher, 2005, "Exchange Rates and Fundamentals: New Evidence from Real-time Data." Journal of International Money and Finance, 24(2), pp.317-341.

Evans, Martin D.D., Richard K. Lyons, 2008, "How Is Macro News Transmitted to Exchange Rates?” Journal of Financial Economics 88(1), pp.26-50.. 
Evans, Martin D.D., Richard K. Lyons, 2005, "Do Currency Markets Absorb News

Quickly?” Journal of International Money and Finance, 24(2), pp.197-217.

Evans, Martin D.D., Richard K. Lyons, 2008, "How is Macro News Transmitted to Exchange Rates?" Journal of Financial Economics, 88(1), pp. 26-50.

Fair, Ray C., 2003, "Shock effects on stocks, bonds, and exchange rates." Journal of International Money and Finance, 22(3), pp.307-341.

Faust, Jon, John H. Rogers, Shing-Yi B. Wang, Jonathan H. Wright, 2007, “The Highfrequency Response of Exchange Rates and Interest Rates to Macroeconomic Announcements." Journal of Monetary Economics, 54(4), pp.1051-1068.

Galati, Gabriele, 2000, "Trading Volumes, Volatility and Spreads in Foreign Exchange Markets: Evidence from Emerging Market Countries.” BIS Working Papers, No. 93.

Galati, Gabriele, Corrinne Ho, 2003, "Macroeconomic News and Euro/Dollar Exchange Rate." Economic Notes by Banca Monte dei Paschi di Siena SpA, 32(3), pp.371 - 398.

Hirshleifer, D., 2001, "Investor Psychology and Asset Prices." Journal of Finance 56(4), pp.1533-1597.

International Monetary Fund. "Annual Report on Exchange Arrangements and Exchange Restrictions.” IMF, 2000 2007.

Laakkonen, H., 2004, "The impact of macroeconomic news on exchange rate volatility." Bank of Finland Discussion Paper 24.

Lui, Yu-Hon, David Mole, 1998, "The Use of Fundamental and Technical Analyses by Foreign Exchange Dealers : Hong Kong Evidence." Journal of International Money and Finance, 17(3), pp.535-545.

McKenzie, Michael, 2002, "The Economics of Exchange Rate Volatility Asymmetry." International Journal of Finance and Economics, 7(3), pp.247-260.

Mian, G. M., S. Sankaraguruswamy, 2008, "Investor Sentiment and Stock Market Response to Corporate News." National University of Singapore working paper.

Tanner, Glenn, 1997, “A Note on Economic News and Intraday Exchange Rates," Journal of Banking and Finance, 21(4), pp.573-585.

Veronesi, Pietro, 1999, "Stock Market Overreaction to Bad News in Good Times: A Rational Expectations Equilibrium Model.” The Review of Financial Studies, 12(5), pp.975-1007. 
Wongswan, Jon, 2006, "Transmission of Information across International Equity Markets." The Review of Financial Studies, 19(4), pp. 1157-1189. 
Table 1. U.S. and National News Announcements

\begin{tabular}{|c|c|c|c|c|c|c|}
\hline & News Announcements & Source & No of Obs & Start Date & Final Date & Time $^{9}$ \\
\hline & United States & & & & & \\
\hline 1 & Business Inventories $^{10}$ & US treasury & 83 & 14-Jan-00 & 13-Dec-06 & $15: 00$ \\
\hline 2 & Budget Deficit $^{11}$ & BEA & 83 & 21-Jan-00 & 12-Dec-06 & 19:00 \\
\hline 3 & Current Account $^{12}$ & Federal reserve & 27 & 15-Mar-00 & 18-Dec-06 & $13: 30$ \\
\hline 4 & Capacity Utilization $^{13}$ & Conference board & 70 & 14-Jan-00 & 15-Dec-06 & $14: 15$ \\
\hline 5 & Consumer Confidence & Federal reserve & 84 & 25-Jan-00 & 28-Dec-06 & $15: 00$ \\
\hline 6 & Consumer Credit & Census & 84 & 7-Jan-00 & 7-Dec-06 & $20: 00$ \\
\hline 7 & Construction Spending & BLS & 84 & 4-Jan-00 & 1-Dec-06 & $15: 00$ \\
\hline 8 & Consumer Price Index ${ }^{14}$ & Census & 82 & $18-F e b-00$ & 15-Dec-06 & $13: 30$ \\
\hline 9 & Durable Goods Orders & Census & 84 & 27-Jan-00 & 22-Dec-06 & $13: 30$ \\
\hline 10 & Factory Orders & BEA & 84 & 5-Jan-00 & 5-Dec-06 & $15: 00$ \\
\hline 11 & Gross Domestic Product & dept of commerce & 84 & 28-Jan-00 & 21-Dec-06 & $13: 30$ \\
\hline 12 & Housing Starts ${ }^{15}$ & BLS & 83 & 19-Jan-00 & 19-Dec-06 & $13: 30$ \\
\hline 13 & Imports $^{16}$ & Federal reserve & 80 & 12-Jan-00 & 14-Dec-06 & $13: 30$ \\
\hline 14 & Interest rate (FOMC) & Federal reserve & 56 & 2-Feb-00 & 12-Dec-06 & $19: 15$ \\
\hline 15 & Industrial production ${ }^{17}$ & ISM & 84 & 14-Jan-00 & 15-Dec-06 & $14: 15$ \\
\hline 16 & NAPM & Conference board & 84 & 3-Jan-00 & 1-Dec-06 & $15: 00$ \\
\hline 17 & Leading Indicators $^{18}$ & Census & 83 & 2-Feb-00 & 21-Dec-06 & $15: 00$ \\
\hline 18 & New Home Sales ${ }^{19}$ & BLS & 84 & 6-Jan-00 & 27-Dec-06 & $15: 00$ \\
\hline 19 & Nonfarm Payroll Employment & BEA & 84 & 7-Jan-00 & 8-Dec-06 & $13: 30$ \\
\hline 20 & Personal Spending & dept of commerce & 60 & 31-Jan-02 & 22-Dec-06 & $13: 30$ \\
\hline 21 & Personal Income & BLS & 84 & 31-Jan-00 & 22-Dec-06 & $13: 30$ \\
\hline 22 & Producer Price & Census & 84 & 13-Jan-00 & 19-Dec-06 & $13: 30$ \\
\hline 23 & Retail Sales ${ }^{20}$ & Census & 83 & 13-Jan-00 & 13-Dec-06 & $13: 30$ \\
\hline 24 & Trade Balance & dept. of Labor & 84 & 20-Jan-00 & 12-Dec-06 & $13: 30$ \\
\hline 25 & Initial Unemployment ${ }^{21}$ & Census & 363 & 6-Jan-00 & 28-Dec-06 & $13: 30$ \\
\hline 26 & Wholesales & Census & 84 & 11-Jan-00 & 11-Dec-06 & $15: 00$ \\
\hline
\end{tabular}

\footnotetext{
${ }^{9}$ The time presented in this table is based on GMT time.

${ }^{10} 3 / 04$ is a missing observation.

${ }^{11} 3 / 04$ is a missing observation.

${ }^{12} 1^{\text {st }}$ Quarter of 04 is a missing observation.

${ }^{13} 1 / 01 \sim 11 / 01,8 / 02,3 / 04,8 / 04$ are missing observations.

${ }_{15}^{14} 1 / 00,8 / 04$ are missing observations.

${ }^{15} 8 / 04$ are missing observation.

${ }^{16} 3 / 00,4 / 01,10 / 01$, and 3/04 are missing observations.

${ }_{17}^{17} 3 / 04$ and 8/04 are missing observations. 9/06 and 12/06 have revisited observations.

${ }^{18} 8 / 04$ is a missing observation.

${ }^{19} 1 / 01$ has a revised observation. $1 / 04$ is a missing observation.

${ }^{20} 3 / 04$ is a missing observation.

${ }^{21} 8 / 21 / 2004$ and $3 / 13 / 2004$ are missing observations.
} 
Table 1. Continued

\begin{tabular}{|c|c|c|c|c|c|c|c|}
\hline & News Announcements & Source & No. & of Obs & Start Date & Final Date & Time \\
\hline & \multicolumn{7}{|l|}{ Czech Republic } \\
\hline 27 & Budget Deficit $^{22}$ & MoF & & 15 & 2-May-00 & 1-Apr-05 & $13: 00$ \\
\hline 28 & Current Account ${ }^{23}$ & $\mathrm{CNB}$ & & 42 & 16-Jun-03 & 13-Dec-06 & 9:00 \\
\hline 29 & Current Account(US Dollar) ${ }^{24}$ & $\mathrm{CNB}$ & & 13 & 5-Jun-01 & 6-Sep-05 & $8: 00$ \\
\hline 30 & Consumer Price Index & $\mathrm{CSO}$ & & 84 & 10-Jan-00 & 8-Dec-06 & $8: 00$ \\
\hline 31 & Exports $^{25}$ & $\mathrm{CSO}$ & & 15 & 23-Jun-03 & 3-Jun-05 & $7: 00$ \\
\hline 32 & Gross Domestic Product & $\mathrm{CSO}$ & & 29 & 22-Mar-00 & 8-Dec-06 & 8:00 \\
\hline 33 & Imports $^{26}$ & $\mathrm{CSO}$ & & 31 & 21-Jan-00 & 3-Jun-05 & $7: 00$ \\
\hline 34 & Industrial production ${ }^{27}$ & $\mathrm{CSO}$ & & 82 & 11-Jan-00 & 12-Dec-06 & 8:00 \\
\hline 35 & Money Supply & $\mathrm{CNB}$ & & 14 & 31-Mar-00 & 30-Apr-01 & 8:00 \\
\hline 36 & Producer Price ${ }^{28}$ & $\mathrm{CSO}$ & & 81 & 13-Jan-00 & 14-Dec-06 & $8: 00$ \\
\hline 37 & Retail Sales ${ }^{29}$ & $\mathrm{CSO}$ & & 83 & 14-Jan-00 & 18-Dec-06 & $8: 00$ \\
\hline 38 & Trade Balance $^{30}$ & $\mathrm{CSO}$ & & 82 & 21-Jan-00 & 6-Dec-06 & $8: 00$ \\
\hline \multirow[t]{2}{*}{39} & Initial Unemployment ${ }^{31}$ & MoL & & 73 & 10-Jan-00 & 12-Jul-06 & $7: 00$ \\
\hline & Hungary & & & & & & \\
\hline 40 & Budget Deficit ${ }^{32}$ & HFM & & 25 & 4-Aug-03 & 8-Aug-06 & $15: 00$ \\
\hline 41 & Current Account ${ }^{33}$ & MNB & & 53 & 3-Apr-00 & 29-Sep-06 & $6: 30$ \\
\hline 42 & Consumer Price Index ${ }^{34}$ & HSO & & 79 & 14-Jan-00 & 12-Dec-06 & $8: 00$ \\
\hline 43 & Gross Domestic Product ${ }^{35}$ & $\mathrm{HSO}$ & & 28 & 31-Mar-00 & 14-Nov-06 & $8: 00$ \\
\hline 44 & Industrial production ${ }^{36}$ & HSO & & 50 & 4-Feb-00 & 13-Oct-06 & $7: 00$ \\
\hline 45 & Producer Price ${ }^{37}$ & $\mathrm{HSO}$ & & 45 & 1-Mar-00 & 30-Nov-06 & 8:00 \\
\hline 46 & Trade Balance $^{38}$ & $\mathrm{HSO}$ & & 34 & 10-Oct-02 & 9-Nov-06 & $8: 00$ \\
\hline
\end{tabular}

${ }^{22} 6 / 01 \sim 12 / 01,1 / 04 \sim 11 / 04,1 / 05 \sim 3 / 05$, and 5/05 12/05 are missing observations.

${ }^{23} 3 / 04$ is a missing observation.

${ }^{24} 3 \mathrm{Q} / 01,2 \mathrm{Q} / 03 \sim 4 \mathrm{Q} / 03$, and $3 \mathrm{Q} / 04$ are missing observations.

${ }^{25} 7 / 03,12 / 03,1 / 04,6 / 04,7 / 04,9 / 04,10 / 04$, and 1/05 3/05 are missing observations.

${ }^{26} 5 / 01 \sim 12 / 01,1 / 02 \sim 12 / 02,1 / 03 \sim 5 / 03,7 / 03,12 / 03,1 / 04,6 / 04,7 / 04,9 / 04,10 / 04$, and 1/05 3/05 are missing observations.

${ }^{27} 9 / 02$ and $3 / 04$ are missing observations.

${ }^{28} 11 / 02,3 / 04$, and $9 / 04$ are missing observations.

${ }^{29} 8 / 04$ is a missing observation.

${ }^{30} 6 / 04$ and $11 / 04$ are missing observations.

${ }^{31} 1 / 06 \sim 6 / 06$ are missing observations.

${ }^{32} 9 / 03 \sim 1 / 04,3 / 04 \sim 5 / 04,1 / 05,2 / 06,3 / 06$, and 5/06 are missing observations.

${ }^{33}$ Current Account is announced quarterly since 2005. 12/01, 3/02,4/02,8/02,12/02, 3/04, 7/04, 8/04, 10/04, $11 / 04$ and $1 \mathrm{Q} / 06$ are missing observations.

${ }^{34} 7 / 00,11 / 00,4 / 01,3 / 03,3 / 03$ are missing observations.

${ }^{35} 4 \mathrm{Q} / 01,1 \mathrm{Q} / 03,2 \mathrm{Q} / 03$, and $4 \mathrm{Q} / 06$ are missing observations.

${ }^{36} 1 / 00,8 / 00 \sim 11 / 00,3 / 01,12 / 01 \sim 4 / 02,6 / 02 \sim 8 / 02,11 / 02 \sim 3 / 03,6 / 03 \sim 6 / 04,2 / 05,2 / 06$ are missing observations.

${ }^{37} 1 / 00,7 / 00,9 / 00,10 / 00,12 / 00,2 / 01,9 / 01,12 / 01,2 / 02,3 / 02,11 / 02,1 / 03 \sim 3 / 03,5 / 03 \sim 3 \sim 04,5 / 04 \sim 7 / 04,9 / 04$, $12 / 04,3 / 05,7 / 05,9 / 05 \sim 12 / 05,2 / 06,5 / 06,7 / 06,10 / 06$ are missing observations.

${ }^{38} 11 / 02,12 / 02,1 / 03 \sim 3 / 03,6 / 03,8 / 03 \sim 1 / 04,4 / 04,5 / 04,7 / 04,6 / 06$ are missing observations. 
Table 1. Continued

\begin{tabular}{|c|c|c|c|c|c|c|}
\hline & News Announcements & Source & No of Obs & Start Date & Final Date & Time \\
\hline & Indonesia & & & & & \\
\hline 47 & Exports ${ }^{39}$ & BPS & 75 & 1-Sep-00 & 1-Dec-06 & $7: 00$ \\
\hline 48 & Gross Domestic Product & BPS & 24 & 15-Nov-00 & 16-Nov-06 & $7: 00$ \\
\hline 49 & Imports $^{40}$ & BPS & 72 & 1-Sep-00 & 1-Dec-06 & $7: 00$ \\
\hline \multirow[t]{2}{*}{50} & Trade Balance ${ }^{41}$ & BPS & 75 & 1-Sep-00 & 1-Dec-06 & $7: 00$ \\
\hline & Korea & & & & & \\
\hline 51 & Consumer Price Index ${ }^{42}$ & NSO & 62 & 31-Aug-00 & 29-Dec-06 & $4: 30$ \\
\hline 52 & Exports ${ }^{43}$ & $\mathrm{MoC}$ & 53 & 2-Feb-01 & 1-Dec-06 & $1: 00$ \\
\hline 53 & Gross Domestic Product ${ }^{44}$ & BOK & 22 & 22-Aug-00 & 24-Oct-06 & 23:00 \\
\hline 54 & Imports $^{45}$ & $\mathrm{MoC}$ & 53 & 2-Feb-01 & 1-Dec-06 & 1:00 \\
\hline 55 & Industrial production ${ }^{46}$ & $\mathrm{NSO}$ & 56 & 31-Jan-01 & 29-Dec-06 & $4: 30$ \\
\hline \multirow[t]{2}{*}{56} & Initial Unemployment & $\mathrm{NSO}$ & 3 & 18-Apr-05 & 13-Sep-06 & $4: 30$ \\
\hline & Mexico & & & & & \\
\hline 57 & Current Account & Banco de Mexico & 14 & 27-Aug-03 & 24-Nov-06 & $20: 30$ \\
\hline 58 & Consumer Confidence ${ }^{47}$ & INEGI & 38 & 4-Aug-03 & 5-Dec-06 & $20: 30$ \\
\hline 59 & Consumer Price Index ${ }^{48}$ & Banco de Mexico & 47 & 7-Jan-00 & 7-Dec-06 & $20: 30$ \\
\hline 60 & Fixed Invest & INEGI & 33 & 7-Apr-04 & 7-Dec-06 & $20: 30$ \\
\hline 61 & Gross Domestic Product ${ }^{49}$ & INEGI & 27 & $16-$ Feb-00 & 22-Nov-06 & $20: 30$ \\
\hline 62 & Industrial production ${ }^{50}$ & INEGI & 81 & 11-Jan-00 & 13-Dec-06 & $20: 30$ \\
\hline 63 & Producer Price ${ }^{51}$ & Banco de Mexico & 29 & 7-Jan-00 & 7-May-04 & $19: 30$ \\
\hline 64 & Retail Sales ${ }^{52}$ & INEGI & 78 & 20-Jan-00 & 19-Dec-06 & $20: 30$ \\
\hline 65 & Trade Balance ${ }^{53}$ & INEGI & 117 & 24-Jan-00 & 26-Dec-06 & $20: 30$ \\
\hline 66 & Unemployment $^{54}$ & INEGI & 80 & 19-Jan-00 & 20-Dec-06 & $20: 30$ \\
\hline 67 & Wholesales & INEGI & 31 & 20-Jan-00 & 22-Jul-02 & $19: 30$ \\
\hline
\end{tabular}

$391 / 02$ is a missing observation.

${ }^{40} 12 / 01,6 / 03,2 / 06,3 / 06$ are missing observations.

$41,12 / 01$ is a missing observation.

$4210 / 00,12 / 00,1 / 01,3 / 01,4 / 01,6 / 01,8 / 01,3 / 02,4 / 02,9 / 03,12 / 03,12 / 04,2 / 05,5 / 05$ are missing observations.

${ }^{43} 4 / 01,1 / 02,3 / 02,4 / 02,6 / 02,11 / 02,12 / 02,1 / 03,2 / 03,5 / 03,6 / 03,8 / 03,9 / 03,12 / 03,2 / 04,12 / 04,1 / 05,2 / 06$ are missing observations.

${ }^{4} 2 \mathrm{Q} / 01,3 \mathrm{Q} / 02,2 \mathrm{Q} / 04$ are missing observations.

${ }^{45} 4 / 01,1 / 02,3 / 02,4 / 02,6 / 02,11 / 02 \sim 2 / 03,5 / 03,6 / 03,8 / 03,9 / 03,12 / 03,2 / 04,12 / 04,2 / 05,2 / 06$ are missing observations.

${ }^{46} 2 / 01,4 / 01 \sim 8 / 01,1 / 02,3 / 02,7 / 02 \sim 10 / 02,2 / 03,12 / 03,12 / 04,12 / 05$ are missing observations.

${ }^{47} 1 / 04,8 / 04,11 / 04$ are missing observations.

$4812 / 01$ is a missing observation.

${ }^{49} 3 \mathrm{Q} / 02,4 \mathrm{Q} / 02$, and $2 \mathrm{Q} / 05$ are missing observations.

${ }^{50} 11 / 02,12 / 02$ and $3 / 04$ are missing observations.

${ }^{51} 12 / 01,6 / 02 \sim 4 / 04$ are missing observations.

$528 / 02,11 / 02,12 / 02,2 / 03,6 / 03$, and $8 / 04$ are missing observations.

${ }^{5} 12 / 01,9 / 02,11 / 02,12 / 02,1 / 03,2 / 03,4 / 03$, and $5 / 03$ are missing observations.

$548 / 02,11 / 02,12 / 02$, and $2 / 03$ are missing observations. 
Table 1. Continued

\begin{tabular}{|c|c|c|c|c|c|c|}
\hline & News Announcements & Source & No of Obs & Start Date & Final Date & Time \\
\hline & Poland & & & & & \\
\hline 68 & Budget Deficit $^{55}$ & MoF & 33 & 15-Nov-01 & 15-Dec-06 & $13: 30$ \\
\hline 69 & Current Account ${ }^{56}$ & NBP & 77 & 3-Apr-00 & 13-Dec-06 & $13: 00$ \\
\hline 70 & Consumer Price Index ${ }^{57}$ & PSO & 79 & $15-$ Feb-00 & 14-Dec-06 & $13: 00$ \\
\hline 71 & Exports $^{58}$ & NBP & 75 & 3-Apr-00 & 13-Dec-06 & $13: 00$ \\
\hline 72 & Gross Domestic Product ${ }^{59}$ & Eurostat & 25 & 21-Jun-00 & 30-Nov-06 & $9: 00$ \\
\hline 73 & Imports $^{60}$ & NBP & 76 & 3-Apr-00 & 13-Dec-06 & $13: 00$ \\
\hline 74 & Money Supply ${ }^{61}$ & NBP & 72 & 14-Apr-00 & 14-Dec-06 & $13: 00$ \\
\hline 75 & Producer Price ${ }^{62}$ & PSO & 77 & 18-Apr-00 & 19-Dec-06 & $13: 00$ \\
\hline 76 & Retail Sales $^{63}$ & PSO & 48 & 20-Dec-02 & 21-Dec-06 & 9:00 \\
\hline 77 & Unemployment $^{64}$ & PSO & 80 & $26-A p r-00$ & 21-Dec-06 & $9: 00$ \\
\hline \multirow[t]{2}{*}{78} & Wholesales $^{65}$ & $\mathrm{PSO}$ & 77 & 18-Apr-00 & 19-Dec-06 & $13: 00$ \\
\hline & South Africa & & & & & \\
\hline 79 & Current Account & SARB & 2 & 21-Sep-06 & 8-Dec-06 & $9: 00$ \\
\hline 80 & Consumer Price Index ${ }^{66}$ & SSA & 83 & 18-Jan-00 & 20-Dec-06 & $9: 30$ \\
\hline 81 & Gross Domestic Product ${ }^{67}$ & SSA & 26 & 28-Feb-00 & 28-Nov-06 & $9: 30$ \\
\hline 82 & Interest rate 68 & SARB & 24 & 15-Nov-01 & 7-Dec-06 & $13: 20$ \\
\hline 83 & Money Supply ${ }^{69}$ & SARB & 79 & 1-Feb-00 & 29-Dec-06 & $6: 00$ \\
\hline 84 & Producer Price ${ }^{70}$ & SSA & 82 & 26-Jan-00 & 21-Dec-06 & $9: 30$ \\
\hline 85 & Retail Sales & SSA & 4 & 4-Nov-04 & 6-Dec-06 & $9: 00$ \\
\hline 86 & Trade Balance $^{71}$ & SARB & 81 & 31-Jan-00 & 28-Dec-06 & $12: 00$ \\
\hline
\end{tabular}

${ }_{55} 12 / 01,1 / 04,3 / 04,2 / 06$, and 9/06 are missing observations.

${ }^{56} 8 / 03,10 / 03,11 / 03,2 / 04$, and $3 / 04$ are missing observations.

${ }^{57} 2 / 01 \sim 4 / 01$, and $3 / 04$ are missing observations.

${ }^{58} 8 / 03,10 / 03,11 / 03,2 / 04,3 / 04,11 / 05$, and $12 / 05$ are missing observations.

${ }^{59} 2 \mathrm{Q} / 00,3 \mathrm{Q} / 00$, and $4 \mathrm{Q} 03$ are missing observations.

${ }^{60} 8 / 03,10 / 03,11 / 03,2 / 04,3 / 04$, and $12 / 05$ are missing observations.

${ }^{61} 10 / 01,5 / 02,8 / 02,3 / 04,4 / 05,8 / 05 \sim 10 / 05,12 / 05$ are missing observations.

${ }^{62} 2 / 01 \sim 4 / 01$, and $8 / 04$ are missing observations.

${ }^{63} 2 / 03$ is a missing observation.

${ }^{64} 10 / 04$ is a missing observation.

${ }^{65} 2 / 01 \sim 4 / 01$, and $8 / 04$ are missing observations.

${ }^{66} 9 / 04$ is a missing observation.

${ }^{67} 4 \mathrm{Q} / 00$, and $3 \mathrm{Q} / 02$ are missing observations.

${ }^{68}$ Bimonthly announcements. 2/01 2/03 are missing observations.

${ }^{69} 7 / 02,12 / 02,2 / 03,3 / 03$, and $2 / 05$ are missing observations.

${ }^{70} 5 / 03$ and $8 / 03$ are missing observations.

${ }^{71} 12 / 00,2 / 05$ and 2/06 are missing observations. 
Table 1. Continued

\begin{tabular}{|c|c|c|c|c|c|c|}
\hline & News Announcements & Source & No of Obs & Start Date & Final Date & Time \\
\hline & Thailand & & & & & \\
\hline 87 & Current Account $^{72}$ & $\begin{array}{l}\text { BOT } \\
\text { Commerce }\end{array}$ & 27 & 30-Sep-04 & 29-Dec-06 & $7: 30$ \\
\hline 88 & Consumer Price Index ${ }^{73}$ & Ministry & 31 & 3-Nov-03 & 1-Dec-06 & $3: 30$ \\
\hline 89 & $\begin{array}{l}\text { Exports }{ }^{74} \\
\text { Gross Domestic }\end{array}$ & BOT & 7 & 30-Sep-04 & 31-May-05 & $8: 00$ \\
\hline 90 & Product ${ }^{75}$ & BOT & 22 & 19-Jun-00 & 4-Dec-06 & $2: 30$ \\
\hline 91 & Interest rate & BOT & 9 & 19-Oct-05 & 13-Dec-06 & $7: 30$ \\
\hline & Turkey & & & & & \\
\hline 92 & Current Account $^{76}$ & CBT & 27 & 24-Jun-04 & 11-Dec-06 & $14: 35$ \\
\hline 93 & Consumer Price Index ${ }^{77}$ & SIS & 48 & 3-Jan-00 & 4-Dec-06 & $14: 30$ \\
\hline 94 & $\begin{array}{l}\text { Exports }{ }^{78} \\
\text { Gross Domestic }\end{array}$ & SIS & 8 & 31-Mar-05 & 29-Jul-06 & $13: 30$ \\
\hline 95 & Product ${ }^{79}$ & SIS & 24 & 31-Aug-00 & 11-Dec-06 & $8: 00$ \\
\hline 96 & Imports $^{80}$ & SIS & 8 & 31-Mar-05 & 29-Jul-06 & $13: 30$ \\
\hline 97 & Industrial production ${ }^{81}$ & SIS & 71 & 8-Aug-00 & 8-Dec-06 & $8: 00$ \\
\hline 98 & Producer Price & SIS & 23 & 3-Feb-05 & 4-Dec-06 & $14: 30$ \\
\hline 99 & Trade Balance ${ }^{82}$ & SIS & 32 & 24-Jun-02 & 29-Dec-06 & $14: 30$ \\
\hline 100 & Unemployment ${ }^{83}$ & SIS & 10 & 9-Dec-04 & 20-Nov-06 & $8: 00$ \\
\hline
\end{tabular}

${ }^{72} 4 / 05$ is a missing observation.

${ }^{73} 12 / 03 \sim 6 / 04$ are missing observations.

${ }^{74} 2 / 05$, and $4 / 05$ are missing observations.

${ }_{75} 3 \mathrm{Q} / 02 \sim 2 \mathrm{Q} / 03,4 \mathrm{Q} / 04$ are missing observations.

${ }^{76} 8 / 04,9 / 04,11 / 04$, and $1 / 06$ are missing observations.

77 2/01 6/03, and 8/03 2/04 are missing observations.

${ }^{78} 10 / 05 \sim 6 / 06$ are missing observations.

${ }^{79} 4 \mathrm{Q} / 01$, and $3 \mathrm{Q} 02$ are missing observations.

${ }^{80} 10 / 05 \sim 6 / 06$ are missing observations.

${ }^{81} 12 / 00,3 / 01,4 / 01,6 / 01,8 / 01$, and $12 / 02$ are missing observations.

${ }^{82} 1 / 04,2 / 04,4 / 04,5 / 04,8 / 04$ are missing observations.

${ }^{83} 1 / 05 \sim 4 / 05,7 / 05,9 / 05,12 / 05,2 / 06 \sim 5 / 06$, and 7/06 9/06 are missing observations. 
Table 2. U.S. and Domestic News Response and R squares

\begin{tabular}{|c|c|c|c|c|c|c|c|c|c|c|c|c|c|c|c|c|c|c|c|c|}
\hline & \multicolumn{2}{|c|}{ Euro } & \multicolumn{2}{|c|}{$\begin{array}{l}\text { Czech } \\
\text { Republic }\end{array}$} & \multicolumn{2}{|c|}{ Hungary } & \multicolumn{2}{|c|}{ Indonesia } & \multicolumn{2}{|c|}{ Korea } & \multicolumn{2}{|c|}{ Mexico } & \multicolumn{2}{|c|}{ Poland } & \multicolumn{2}{|c|}{ South Africa } & \multicolumn{2}{|c|}{ Thailand } & \multicolumn{2}{|c|}{ Turkey } \\
\hline & $\beta$ & $\mathbf{R}^{2}$ & $\beta$ & $\mathbf{R}^{2}$ & $\beta$ & $\mathbf{R}^{2}$ & $\beta$ & $\mathbf{R}^{2}$ & $\beta$ & $\mathbf{R}^{2}$ & $\beta$ & $\mathbf{R}^{2}$ & $\beta$ & $\mathbf{R}^{2}$ & $\beta$ & $\mathbf{R}^{2}$ & $\beta$ & $\mathbf{R}^{2}$ & $\beta$ & $\mathbf{R}^{2}$ \\
\hline \multicolumn{21}{|l|}{ U.S. Announcement } \\
\hline Business Inventories & 0.00 & 0.00 & 0.01 & 0.00 & -0.01 & 0.00 & 0.01 & 0.03 & 0.00 & 0.00 & -0.01 & 0.00 & -0.01 & 0.00 & -0.03 & 0.03 & 0.00 & 0.00 & -0.03 & 0.02 \\
\hline Budget Deficit & 0.00 & 0.01 & -0.01 & 0.02 & 0.01 & 0.00 & 0.01 & 0.00 & $0.17^{*}$ & 0.15 & 0.00 & 0.01 & 0.00 & 0.00 & -0.01 & 0.01 & -0.03 & 0.02 & 0.08 & 0.66 \\
\hline Current Account & 0.01 & 0.06 & $0.04^{*}$ & 0.15 & 0.05 & 0.13 & $-0.07^{*}$ & 0.21 & 0.01 & 0.02 & 0.00 & 0.00 & 0.02 & 0.10 & 0.04 & 0.09 & 0.03 & 0.15 & 0.04 & 0.04 \\
\hline Capital Utilization & $0.01^{*}$ & 0.10 & 0.01 & 0.02 & 0.00 & 0.00 & -0.01 & 0.01 & -0.03 & 0.04 & 0.00 & 0.01 & 0.00 & 0.00 & $0.06^{\star *}$ & 0.13 & 0.01 & 0.02 & 0.02 & 0.03 \\
\hline Consumer Confidence & $0.06^{* *}$ & 0.38 & $0.11^{* *}$ & 0.48 & $0.10^{* *}$ & 0.40 & 0.01 & 0.02 & 0.03 & 0.07 & $-0.03^{* *}$ & 0.13 & $0.05^{\star *}$ & 0.17 & $0.05^{* *}$ & 0.18 & 0.00 & 0.00 & $0.04^{*}$ & 0.09 \\
\hline Consumer Credit & 0.00 & 0.01 & 0.00 & 0.00 & $-0.02^{*}$ & 0.06 & -0.01 & 0.04 & -0.01 & 0.07 & -0.01 & 0.02 & 0.00 & 0.00 & -0.02 & 0.02 & 0.01 & 0.01 & -0.05 & 0.59 \\
\hline Construction Spending & 0.00 & 0.00 & $0.04^{*}$ & 0.08 & 0.04 & 0.05 & 0.00 & 0.00 & 0.01 & 0.01 & 0.00 & 0.00 & 0.02 & 0.05 & -0.01 & 0.00 & -0.02 & 0.05 & -0.02 & 0.01 \\
\hline Consumer Price Index & 0.01 & 0.04 & 0.01 & 0.00 & 0.02 & 0.02 & $-0.05^{\star *}$ & 0.13 & 0.00 & 0.00 & $0.03^{*}$ & 0.05 & 0.02 & 0.01 & 0.05 & 0.04 & 0.01 & 0.00 & 0.06 & 0.04 \\
\hline Durable Goods Orders & $0.02^{* *}$ & 0.09 & $0.06^{* *}$ & 0.21 & $0.07^{* *}$ & 0.22 & $0.05^{*}$ & 0.13 & 0.02 & 0.06 & -0.01 & 0.02 & $0.04^{* *}$ & 0.14 & $0.05^{* *}$ & 0.11 & -0.01 & 0.00 & -0.02 & 0.01 \\
\hline Factory Orders & 0.00 & 0.00 & 0.03 & 0.05 & 0.02 & 0.03 & 0.00 & 0.00 & -0.01 & 0.01 & 0.00 & 0.00 & 0.00 & 0.00 & $0.03^{*}$ & 0.06 & 0.01 & 0.03 & $0.05^{\star *}$ & 0.17 \\
\hline Gross Domestic Product & $0.05^{* *}$ & 0.27 & $0.11^{* *}$ & 0.42 & $0.11^{* *}$ & 0.39 & $0.06^{* *}$ & 0.29 & 0.02 & 0.06 & 0.00 & 0.00 & $0.07^{* *}$ & 0.37 & $0.07^{* *}$ & 0.16 & 0.00 & 0.00 & 0.01 & 0.00 \\
\hline Housing Starts & 0.00 & 0.00 & 0.01 & 0.01 & 0.01 & 0.01 & 0.00 & 0.00 & 0.02 & 0.02 & 0.00 & 0.00 & 0.01 & 0.00 & 0.01 & 0.01 & -0.01 & 0.01 & 0.01 & 0.00 \\
\hline Imports & -0.01 & 0.01 & $0.03^{*}$ & 0.07 & 0.02 & 0.04 & 0.01 & 0.01 & 0.03 & 0.07 & 0.02 & 0.01 & 0.03 & 0.04 & 0.00 & 0.00 & -0.01 & 0.01 & -0.02 & 0.01 \\
\hline Interest rate & 0.00 & 0.00 & 0.01 & 0.00 & 0.00 & 0.00 & 0.00 & 0.00 & 0.00 & 0.00 & 0.00 & 0.00 & 0.00 & 0.00 & 0.00 & 0.00 & 0.01 & 0.00 & 0.00 & 0.00 \\
\hline Industrial production & 0.01 & 0.01 & $0.02^{*}$ & 0.06 & 0.02 & 0.04 & 0.00 & 0.00 & 0.00 & 0.00 & -0.01 & 0.01 & 0.01 & 0.02 & 0.02 & 0.01 & 0.00 & 0.00 & 0.01 & 0.00 \\
\hline NAPM & 0.17 & 0.02 & $1.39^{\star *}$ & 0.21 & $1.35^{\star *}$ & 0.16 & 0.20 & 0.01 & 0.29 & 0.02 & 0.04 & 0.00 & $0.82^{* *}$ & 0.19 & 0.44 & 0.03 & $-0.44^{*}$ & 0.09 & -0.28 & 0.01 \\
\hline Leading Indicators & 0.00 & 0.00 & 0.00 & 0.00 & 0.02 & 0.04 & -0.01 & 0.02 & -0.01 & 0.01 & 0.00 & 0.00 & 0.00 & 0.00 & -0.01 & 0.00 & 0.01 & 0.01 & 0.01 & 0.00 \\
\hline New Home Sales & $0.01^{*}$ & 0.05 & $0.04^{*}$ & 0.07 & $0.05^{\star \star}$ & 0.13 & $0.03^{*}$ & 0.14 & 0.03 & 0.06 & 0.01 & 0.01 & $0.03^{* *}$ & 0.14 & 0.01 & 0.00 & -0.01 & 0.02 & -0.01 & 0.00 \\
\hline Nonfarm Payroll & $0.09^{* *}$ & 0.18 & $0.22^{\star \star}$ & 0.34 & $0.24^{* *}$ & 0.36 & $0.06^{*}$ & 0.12 & $0.09^{*}$ & 0.16 & 0.03 & 0.03 & $0.18^{* *}$ & 0.31 & $0.19^{* *}$ & 0.24 & 0.01 & 0.01 & 0.02 & 0.00 \\
\hline Personal Spending & 0.00 & 0.00 & 0.01 & 0.00 & 0.02 & 0.02 & 0.01 & 0.03 & -0.01 & 0.01 & 0.00 & 0.00 & 0.00 & 0.00 & 0.01 & 0.01 & -0.01 & 0.02 & -0.01 & 0.00 \\
\hline Personal Income & 0.01 & 0.04 & 0.00 & 0.00 & 0.01 & 0.00 & -0.01 & 0.02 & 0.00 & 0.00 & 0.01 & 0.01 & 0.00 & 0.00 & 0.01 & 0.00 & 0.00 & 0.00 & 0.02 & 0.01 \\
\hline Producer Price & 0.01 & 0.03 & 0.02 & 0.01 & 0.02 & 0.01 & 0.03 & 0.05 & 0.01 & 0.03 & 0.01 & 0.01 & 0.03 & 0.05 & 0.03 & 0.02 & 0.00 & 0.00 & 0.00 & 0.00 \\
\hline Retail Sales & $0.03^{* *}$ & 0.20 & $0.08^{\star *}$ & 0.22 & $0.08^{* *}$ & 0.24 & 0.03 & 0.06 & $0.03^{*}$ & 0.09 & $-0.05^{*}$ & 0.08 & $0.07^{* *}$ & 0.23 & 0.03 & 0.01 & 0.01 & 0.03 & 0.00 & 0.00 \\
\hline Trade Balance & $0.04^{* *}$ & 0.20 & $0.12^{* *}$ & 0.38 & $0.11^{* *}$ & 0.36 & 0.00 & 0.00 & 0.02 & 0.05 & -0.01 & 0.01 & $0.10^{* *}$ & 0.35 & $0.08^{* *}$ & 0.19 & 0.01 & 0.00 & 0.02 & 0.01 \\
\hline Initial Unemployment & $-0.01^{* *}$ & 0.04 & $-0.03^{* *}$ & 0.07 & $-0.03^{* *}$ & 0.06 & 0.00 & 0.00 & -0.01 & 0.01 & 0.00 & 0.00 & $-0.01^{*}$ & 0.02 & -0.01 & 0.00 & 0.00 & 0.00 & -0.01 & 0.00 \\
\hline Wholesales & 0.01 & 0.04 & 0.00 & 0.00 & 0.00 & 0.00 & 0.00 & 0.00 & 0.00 & 0.00 & -0.01 & 0.01 & 0.00 & 0.00 & 0.01 & 0.01 & 0.00 & 0.00 & -0.01 & 0.01 \\
\hline
\end{tabular}




\section{Table 2. Continued}

\begin{tabular}{|c|c|c|c|c|c|c|c|c|c|c|c|c|c|c|c|c|c|c|c|c|}
\hline & \multicolumn{2}{|c|}{ Euro } & \multicolumn{2}{|c|}{ Czech Republic } & \multicolumn{2}{|c|}{ Hungary } & \multicolumn{2}{|c|}{ Indonesia } & \multicolumn{2}{|c|}{ Korea } & \multicolumn{2}{|c|}{ Mexico } & \multicolumn{2}{|c|}{ Poland } & \multicolumn{2}{|c|}{ South Africa } & \multicolumn{2}{|c|}{ Thailand } & \multicolumn{2}{|c|}{ Turkey } \\
\hline & $\beta$ & $\mathbf{R}^{2}$ & $\beta$ & $\mathbf{R}^{2}$ & $\beta$ & $\mathbf{R}^{2}$ & $\beta$ & $\mathbf{R}^{2}$ & $\beta$ & $\mathbf{R}^{2}$ & $\beta$ & $\mathbf{R}^{2}$ & $\beta$ & $\mathbf{R}^{2}$ & $\beta$ & $\mathbf{R}^{2}$ & $\beta$ & $\mathbf{R}^{2}$ & $\beta$ & $\mathbf{R}^{2}$ \\
\hline \multicolumn{21}{|c|}{ Domestic Announcement } \\
\hline Budget Deficit & & & 0.00 & 0.00 & 0.04 & 0.16 & & & & & & & 0.00 & 0.00 & & & & & & \\
\hline Current Account & & & $-0.03^{* *}$ & 0.24 & -0.02 & 0.04 & & & & & 0.00 & 0.02 & $-0.08^{* *}$ & 0.24 & 0.13 & 0.11 & 0.37 & 0.11 & 0.03 & 0.05 \\
\hline Current Account(US) & & & -0.01 & 0.02 & & & & & & & & & & & & & & & & \\
\hline Consumer Confidence & & & & & & & & & & & 0.00 & 0.00 & & & & & & & & \\
\hline Consumer Price Index & & & -0.02 & 0.03 & 0.02 & 0.03 & & & $0.01^{*}$ & 0.16 & 0.01 & 0.06 & 0.01 & 0.02 & $-0.07^{\star *}$ & 0.28 & 0.00 & 0.00 & -0.01 & 0.00 \\
\hline Exports & & & -0.03 & 0.03 & & & 0.00 & 0.00 & 0.01 & 0.04 & & & 0.01 & 0.00 & & & 0.00 & 0.02 & 0.04 & 0.04 \\
\hline Fixed Invest & & & & & & & & & & & 0.00 & 0.00 & & & & & & & & \\
\hline Gross Domestic Product & & & $-0.03^{*}$ & 0.14 & 0.01 & 0.01 & 0.00 & 0.00 & 0.01 & 0.00 & $-0.03^{*}$ & 0.25 & -0.04 & 0.18 & -0.03 & 0.10 & -0.07 & 0.06 & -0.02 & 0.01 \\
\hline Imports & & & -0.01 & 0.01 & & & -0.01 & 0.03 & 0.00 & 0.00 & & & 0.04 & 0.06 & & & & & -0.14 & 0.30 \\
\hline Interest rate & & & & & & & & & & & & & & & $-0.26^{*}$ & 0.20 & -0.01 & 0.03 & & \\
\hline Industrial production & & & -0.01 & 0.04 & $-0.03^{*}$ & 0.10 & & & 0.00 & 0.00 & 0.01 & 0.01 & & & & & & & -0.01 & 0.00 \\
\hline Money Supply & & & 0.00 & 0.00 & & & & & & & & & -0.01 & 0.03 & 0.04 & 0.07 & & & & \\
\hline Producer Price & & & $-0.02^{* *}$ & 0.10 & -0.02 & 0.08 & & & & & 0.01 & 0.14 & 0.00 & 0.00 & 0.01 & 0.01 & & & 0.15 & 0.15 \\
\hline Retail Sales & & & $-0.03^{* *}$ & 0.10 & & & & & & & -0.01 & 0.05 & -0.01 & 0.01 & -0.02 & 0.05 & & & & \\
\hline Trade Balance & & & $-0.07^{* *}$ & 0.33 & -0.01 & 0.00 & 0.36 & 0.00 & & & $-0.01^{* *}$ & 0.10 & & & $-0.13^{* *}$ & 0.30 & & & 0.04 & 0.03 \\
\hline Initial Unemployment & & & -0.01 & 0.02 & & & & & 0.00 & 0.29 & -0.02 & 0.02 & 0.02 & 0.03 & & & & & -0.01 & 0.01 \\
\hline Wholesales & & & & & & & & & & & $-0.03^{*}$ & 0.25 & -0.02 & 0.04 & & & & & & \\
\hline
\end{tabular}

Notes: We estimate the exchange rate conditional mean model (3) $R_{t}=\beta_{k} S_{k t}+\varepsilon_{t}$, where $R_{t}$ is the 5-minute return from period t to period $t+1$, and $S_{k t}$ is the standardized news surprise as described in the text. We estimate the regression only using non-missing data for each news surprise. $\beta_{k}$ and $\mathrm{R}^{2}$ are reported for each regression result. Asterisks denote statistical significance (*** at 1-percent level, $* *$ at 5 -percent level, and $*$ at 10 -percent level). 
Table 3. The Impact of Major News Surprises on FX Returns and FX Volatility

\begin{tabular}{|c|c|c|c|c|c|c|c|c|c|}
\hline Announcements & $\begin{array}{c}\text { Czech } \\
\text { Republic }\end{array}$ & Hungary & Indonesia & Korea & Mexico & Poland & $\begin{array}{l}\text { South } \\
\text { Africa }\end{array}$ & Thailand & Turkey \\
\hline \multicolumn{10}{|c|}{ Response of Contemporaneous News Surprises on FX Returns } \\
\hline Durable Goods Orders & $0.05^{\star *}$ & $0.06^{* *}$ & $0.04^{* *}$ & $0.03^{*}$ & $-0.01^{*}$ & $0.04^{* *}$ & $0.05^{* *}$ & $-0.03^{* *}$ & -0.01 \\
\hline Nonfarm Payroll & $0.19^{* *}$ & $0.21^{* *}$ & $0.04^{\star *}$ & $0.09^{* *}$ & $0.02^{\star *}$ & $0.18^{* *}$ & $0.18^{* *}$ & 0.00 & $0.02^{*}$ \\
\hline Trade Balance & $0.12^{\star *}$ & $0.12^{* *}$ & 0.00 & $0.02^{* *}$ & $-0.02^{* *}$ & $0.12^{* *}$ & $0.09^{* *}$ & 0.00 & 0.02 \\
\hline Producer Price & $0.02^{* \star}$ & $0.02^{* *}$ & $0.04^{* *}$ & 0.01 & $0.01^{*}$ & $0.02^{* *}$ & $0.05^{* *}$ & 0.00 & -0.01 \\
\hline New Home Sales & $0.02^{* *}$ & $0.03^{* *}$ & $0.03^{* *}$ & $0.03^{* *}$ & $0.01^{*}$ & $0.03^{* *}$ & 0.00 & -0.01 & -0.02 \\
\hline GDP & $0.10^{* *}$ & $0.11^{* *}$ & $0.07^{\star *}$ & $0.02^{* *}$ & 0.00 & $0.07^{\star *}$ & $0.07^{* *}$ & -0.01 & 0.03 \\
\hline Consumer confidence & $0.11^{* *}$ & $0.10^{* *}$ & 0.01 & $0.02^{* *}$ & $-0.03^{* *}$ & $0.04^{* *}$ & $0.05^{* *}$ & 0.00 & 0.05 \\
\hline Retail Sales & $0.07^{* *}$ & $0.07^{* *}$ & $0.03^{* *}$ & $0.03^{* *}$ & $-0.07^{* *}$ & $0.07^{* *}$ & 0.02 & 0.01 & -0.01 \\
\hline Initial Unemployment & $-0.03^{* *}$ & $-0.03^{* *}$ & -0.01 & $-0.01^{* *}$ & 0.00 & $-0.02^{* *}$ & $-0.02^{* *}$ & 0.01 & 0.01 \\
\hline \multicolumn{10}{|c|}{ Impact of Contemporaneous News Surprises on Volatility } \\
\hline Durable Goods Orders & $0.02^{* *}$ & $0.05^{* *}$ & $0.01^{*}$ & $-0.01^{* *}$ & 0.00 & $0.01^{* *}$ & $0.01^{* *}$ & $0.02^{* *}$ & 0.01 \\
\hline Nonfarm Payroll & $0.17^{* *}$ & $0.17^{* *}$ & $-0.01^{* *}$ & $0.09^{* *}$ & $0.05^{* *}$ & $0.16^{* *}$ & $0.18^{* *}$ & $0.01^{* *}$ & $0.10^{* *}$ \\
\hline Trade Balance & $0.04^{* *}$ & $0.04^{* *}$ & $0.04^{* *}$ & $0.04^{* *}$ & $0.00^{* *}$ & $0.06^{\star *}$ & $0.03^{* *}$ & 0.00 & 0.01 \\
\hline Producer Price & $0.03^{* *}$ & $0.03^{* *}$ & $0.02^{* *}$ & $-0.01^{*}$ & $0.01^{* *}$ & 0.01 & $0.01^{* *}$ & 0.00 & $0.07^{* *}$ \\
\hline New Home Sales & $0.03^{* *}$ & $0.03^{* *}$ & $0.02^{* *}$ & $0.02^{* *}$ & 0.00 & $0.03^{* *}$ & 0.00 & 0.00 & -0.01 \\
\hline GDP & $0.05^{* *}$ & $0.04^{* *}$ & $0.04^{* *}$ & $0.04^{* *}$ & 0.00 & $0.04^{* *}$ & $0.04^{* *}$ & 0.01 & $0.03^{* *}$ \\
\hline Consumer confidence & $0.03^{* *}$ & $0.04^{* *}$ & $0.03^{* *}$ & $0.03^{* *}$ & $0.02^{* *}$ & $0.03^{* *}$ & 0.00 & $0.01^{* *}$ & 0.00 \\
\hline Retail Sales & $0.03^{* *}$ & $0.03^{* *}$ & $0.03^{* *}$ & $0.05^{\star \star}$ & $0.03^{\star *}$ & $0.04^{* *}$ & $0.00^{*}$ & 0.00 & 0.00 \\
\hline Initial Unemployment & $0.01^{* *}$ & 0.00 & $0.01^{* *}$ & $0.01^{* *}$ & $0.01^{* *}$ & $0.02^{* *}$ & $0.02^{* *}$ & 0.00 & $0.01^{* *}$ \\
\hline \multicolumn{10}{|c|}{ Cumulated Impact of News Surprises on Volatility } \\
\hline Durable Goods Orders & $0.17^{* *}$ & $0.20^{* *}$ & $0.05^{\star}$ & $0.05^{\star *}$ & 0.00 & $0.07^{* *}$ & $0.13^{* *}$ & $0.02^{* *}$ & 0.03 \\
\hline Nonfarm Payroll & $0.26^{* *}$ & $0.32^{* *}$ & $0.16^{\star *}$ & $0.39^{* *}$ & $0.19^{* *}$ & $0.37^{* *}$ & $0.38^{* *}$ & $0.05^{\star *}$ & $0.16^{* *}$ \\
\hline Trade Balance & $0.05^{\star *}$ & $0.06^{* *}$ & $0.01^{* *}$ & $0.25^{\star *}$ & $0.02^{* *}$ & $0.11^{* *}$ & $0.11^{* *}$ & -0.03 & 0.02 \\
\hline Producer Price & $0.03^{* *}$ & $0.06^{* *}$ & $0.04^{* *}$ & $0.04^{*}$ & $0.01^{* *}$ & 0.01 & $0.10^{* *}$ & -0.01 & $0.08^{* *}$ \\
\hline New Home Sales & $0.10^{\star *}$ & $0.06^{* *}$ & $0.05^{\star *}$ & $0.03^{* *}$ & -0.01 & $0.08^{* *}$ & 0.01 & 0.00 & -0.01 \\
\hline GDP & $0.06^{* *}$ & $0.02^{* *}$ & $0.05^{\star *}$ & $0.04^{* *}$ & 0.00 & $0.11^{* *}$ & $0.08^{* *}$ & 0.03 & $-0.02^{* *}$ \\
\hline Consumer confidence & $0.03^{* *}$ & $0.10^{* *}$ & $0.13^{* *}$ & $0.07^{* *}$ & $0.05^{\star *}$ & $0.12^{* *}$ & 0.01 & $0.02^{* *}$ & -0.04 \\
\hline Retail Sales & $0.09^{* *}$ & $0.05^{* *}$ & $0.06^{* *}$ & $0.13^{* *}$ & $0.03^{* *}$ & $0.16^{* *}$ & $0.06^{*}$ & 0.00 & 0.01 \\
\hline Initial Unemployment & $0.03^{* *}$ & 0.00 & $-0.01^{* *}$ & $0.01^{* *}$ & $0.04^{* *}$ & $0.12^{\star *}$ & $0.07^{* *}$ & 0.00 & $0.01^{* *}$ \\
\hline
\end{tabular}

Notes: We estimate the exchange rate conditional mean model (4) $R_{t}=\beta_{0}+\sum_{i=1}^{I} \beta_{i} R_{t-i}+\sum_{k=1}^{K} \sum_{j=0}^{J} \beta_{k j} S_{k, t-j}+\varepsilon_{t}$, and we report estimates of the contemporaneous response of exchange-rate returns to news surprises, $\beta_{k 0}$. We also estimate the disturbance volatility model (5)

$\left|\hat{\varepsilon}_{t}\right|=c+\psi \frac{\hat{\sigma}_{d(t)}}{\sqrt{288}}+\sum_{k=1}^{K} \sum_{j^{\prime}=0}^{J^{\prime}} \beta_{k j^{\prime}}\left|S_{k, t-j^{\prime}}\right|+\left(\sum_{q=1}^{Q}\left(\delta_{q} \cos \left(\frac{q 2 \pi t}{288}\right)+\phi_{q} \sin \left(\frac{q 2 \pi t}{288}\right)\right)\right)+u_{t}$, and we report estimates of the contemporaneous response of exchange-rate volatility to news surprise, $\beta_{\mathrm{k} 0}=\eta_{\mathrm{k}} \mathrm{p}_{\mathrm{k}}(0)$. In addition, we report estimates of the cumulative volatility response, $\sum_{j^{\prime}=0}^{12} \eta_{k} p_{k}\left(j^{\prime}\right)$, as described in the text. Asterisks denote statistical significance (*** at 1-percent level, $* *$ at 5-percent level, and $*$ at 10 -percent level). 
Table 4. Response of Major News Surprises and Announcement Effects on FX returns

\begin{tabular}{|c|c|c|c|c|c|c|c|c|c|c|}
\hline Announcements & & $\begin{array}{c}\text { Czech } \\
\text { Republic }\end{array}$ & Hungary & Indonesia & Korea & Mexico & Poland & South Africa & Thailand & Turkey \\
\hline \multicolumn{11}{|c|}{ Impact of Major News Surprises on FX Returns } \\
\hline \multirow{2}{*}{$\begin{array}{l}\text { Durable Goods } \\
\text { Orders }\end{array}$} & $\mathrm{B}_{\mathrm{k} 0}$ & $0.06^{\star * *}$ & $0.07^{* * *}$ & $0.05^{* * *}$ & $0.02^{\star * *}$ & $-0.02^{* * *}$ & $0.05^{\star * *}$ & $0.05^{\star * *}$ & $-0.03^{* * *}$ & -0.01 \\
\hline & $\Theta_{\mathrm{k} 0}$ & -0.01 & $-0.02^{* * *}$ & 0.00 & 0.01 & $0.01^{* *}$ & $-0.02^{* * *}$ & -0.02 & $0.03^{* * *}$ & $0.04^{*}$ \\
\hline \multirow{2}{*}{ Nonfarm Payroll } & $\mathrm{B}_{\mathrm{k} 0}$ & $0.22^{* * *}$ & $0.25^{\star * *}$ & $0.06^{* * *}$ & $0.09^{* * *}$ & $0.01^{* * *}$ & $0.20^{* * *}$ & $0.18^{* * *}$ & 0.01 & $0.05^{* * *}$ \\
\hline & $\Theta_{\mathrm{k} 0}$ & $0.05^{* * *}$ & $0.06^{* * *}$ & $0.02^{* * *}$ & $0.04^{* * *}$ & $-0.05^{\star * *}$ & $0.02^{* * *}$ & -0.01 & 0.01 & $0.10^{* * *}$ \\
\hline \multirow{2}{*}{ Trade Balance } & $\mathrm{B}_{\mathrm{k} 0}$ & $0.12^{* * *}$ & $0.11^{* * *}$ & 0.00 & $0.02^{* * *}$ & $-0.02^{* * *}$ & $0.11^{* * *}$ & $0.10^{* * *}$ & 0.00 & $0.03^{*}$ \\
\hline & $\Theta_{\mathrm{k} 0}$ & 0.01 & 0.00 & $-0.02^{* *}$ & $0.02^{\star \star *}$ & 0.00 & $0.01^{* *}$ & 0.01 & 0.01 & $0.05^{* *}$ \\
\hline \multirow{2}{*}{ Producer Price } & $\mathrm{B}_{\mathrm{k} 0}$ & 0.00 & 0.01 & $0.03^{* * *}$ & $0.02^{* * *}$ & $0.01^{* *}$ & $0.01^{* *}$ & $0.04^{* * *}$ & 0.01 & 0.00 \\
\hline & $\Theta_{\mathrm{k} 0}$ & $-0.02^{* * *}$ & $-0.03^{* * *}$ & $-0.02^{* *}$ & -0.01 & $-0.03^{* * *}$ & $-0.02^{* * *}$ & 0.00 & 0.01 & $0.09^{* * *}$ \\
\hline \multirow{2}{*}{$\begin{array}{l}\text { New Home } \\
\text { Sales }\end{array}$} & $\mathrm{B}_{\mathrm{k} 0}$ & $0.02^{* * *}$ & $0.03^{* * *}$ & $0.02^{* * *}$ & $0.03^{* * *}$ & $0.01^{* *}$ & $0.03^{* * *}$ & 0.00 & -0.01 & -0.03 \\
\hline & $\Theta_{\mathrm{k} 0}$ & $-0.01^{* *}$ & $0.03^{* * *}$ & 0.01 & $-0.02^{* * *}$ & $-0.01^{* *}$ & 0.01 & 0.01 & -0.01 & 0.03 \\
\hline \multirow{2}{*}{$\begin{array}{l}\text { Gross Domestic } \\
\text { Product }\end{array}$} & $\mathrm{B}_{\mathrm{k} 0}$ & $0.10^{* * *}$ & $0.11^{* * *}$ & $0.06^{* * *}$ & $0.02^{* * *}$ & 0.00 & $0.07^{\star * *}$ & $0.07^{* * *}$ & 0.00 & $0.03^{*}$ \\
\hline & $\Theta_{\mathrm{k} 0}$ & $-0.03^{* * *}$ & $-0.04^{* * *}$ & $-0.04^{* * *}$ & $-0.03^{* * *}$ & $-0.01^{* *}$ & -0.01 & $-0.02^{*}$ & 0.01 & $0.07^{* * *}$ \\
\hline \multirow{2}{*}{$\begin{array}{l}\text { Consumer } \\
\text { Confidence }\end{array}$} & $\mathrm{B}_{\mathrm{k} 0}$ & $0.11^{* * *}$ & $0.10^{* * *}$ & 0.01 & 0.01 & $-0.03^{* * *}$ & $0.05^{\star * *}$ & $0.05^{\star \star *}$ & 0.00 & 0.05 \\
\hline & $\Theta_{k 0}$ & 0.00 & -0.01 & -0.01 & $0.04^{* * *}$ & $0.01^{* *}$ & $-0.02^{* * *}$ & 0.00 & 0.00 & -0.02 \\
\hline \multirow{2}{*}{ Retail Sales } & $\mathrm{B}_{\mathrm{k} 0}$ & $0.09^{* * *}$ & $0.08^{* * *}$ & $0.03^{* * *}$ & $0.03^{* * *}$ & $-0.07^{* * *}$ & $0.08^{* * *}$ & $0.03^{* *}$ & 0.01 & -0.01 \\
\hline & $\Theta_{\mathrm{k} 0}$ & 0.00 & 0.00 & 0.00 & 0.00 & $0.02^{* * *}$ & 0.00 & $-0.06^{* * *}$ & 0.01 & $-0.04^{* *}$ \\
\hline \multirow{2}{*}{$\begin{array}{l}\text { Initial } \\
\text { Unemployment }\end{array}$} & $\mathrm{B}_{\mathrm{k} 0}$ & $-0.04^{* * *}$ & $-0.03^{* * *}$ & $-0.01^{* *}$ & $-0.02^{* * *}$ & 0.00 & $-0.02^{* * *}$ & $-0.02^{* * *}$ & 0.01 & 0.01 \\
\hline & $\Theta_{k 0}$ & $-0.01^{* * *}$ & $-0.01^{* *}$ & 0.00 & 0.00 & 0.00 & $-0.01^{* * *}$ & -0.01 & $-0.01^{*}$ & 0.01 \\
\hline \multicolumn{11}{|c|}{ Impact of Major News Surprises on Volatility } \\
\hline \multirow{2}{*}{$\begin{array}{l}\text { Durable Goods } \\
\text { Orders }\end{array}$} & $\mathrm{B}_{\mathrm{k} 0}$ & -0.01 & 0.00 & 0.00 & -0.01 & 0.00 & 0.00 & 0.00 & $0.03^{* * *}$ & 0.01 \\
\hline & $\Theta_{\mathrm{k} 0}$ & $0.05^{* * *}$ & $0.05^{* * *}$ & $0.04^{* * *}$ & $0.03^{* * *}$ & $0.02^{* * *}$ & $0.04^{* * *}$ & 0.01 & 0.00 & -0.01 \\
\hline \multirow{2}{*}{ Nonfarm Payroll } & $\mathrm{B}_{\mathrm{k} 0}$ & $0.07^{* * *}$ & $0.06^{* * *}$ & 0.00 & $0.02^{*}$ & $0.02^{* * *}$ & $0.08^{* * *}$ & $0.12^{* * *}$ & 0.01 & $0.04^{* *}$ \\
\hline & $\Theta_{\mathrm{k} 0}$ & $0.17^{* * *}$ & $0.16^{* * *}$ & $0.08^{* * *}$ & $0.08^{* * *}$ & $0.06^{* * *}$ & $0.12^{\star \star *}$ & $0.08^{* * *}$ & 0.00 & $0.07^{* * *}$ \\
\hline \multirow{2}{*}{ Trade Balance } & $\mathrm{B}_{\mathrm{k} 0}$ & $0.02^{* * *}$ & $0.02^{* * *}$ & 0.01 & $0.03^{\star * *}$ & 0.00 & $0.03^{* * *}$ & $0.03^{* * *}$ & $-0.01^{* *}$ & -0.03 \\
\hline & $\Theta_{\mathrm{k} 0}$ & $0.04^{* * *}$ & $0.04^{* * *}$ & $0.05^{\star * *}$ & 0.00 & $0.02^{* *}$ & $0.04^{* * *}$ & 0.02 & $0.03^{\star \star *}$ & $0.04^{*}$ \\
\hline \multirow{2}{*}{ Producer Price } & $\mathrm{B}_{\mathrm{k} 0}$ & $-0.02^{* * *}$ & $-0.02^{* * *}$ & 0.00 & 0.00 & $-0.01^{*}$ & 0.00 & 0.00 & 0.00 & -0.01 \\
\hline & $\Theta_{k 0}$ & $0.06^{* * *}$ & $0.06^{* * *}$ & $0.04^{* * *}$ & 0.02 & $0.03^{* * *}$ & $0.03^{* *}$ & $0.04^{* * *}$ & -0.01 & $0.08^{* * *}$ \\
\hline \multirow{2}{*}{$\begin{array}{l}\text { New Home } \\
\text { Sales }\end{array}$} & $\mathrm{B}_{\mathrm{k} 0}$ & $-0.02^{* * *}$ & $-0.02^{* *}$ & 0.00 & -0.01 & 0.00 & 0.01 & 0.00 & 0.00 & 0.01 \\
\hline & $\Theta_{\mathrm{k} 0}$ & $0.07^{* * *}$ & $0.05^{\star \star *}$ & $0.03^{\star *}$ & $0.06^{\star \star *}$ & 0.01 & $0.02^{* * *}$ & $0.03^{* * *}$ & 0.00 & -0.03 \\
\hline \multirow{2}{*}{$\begin{array}{l}\text { Gross Domestic } \\
\text { Product }\end{array}$} & $\mathrm{B}_{\mathrm{k} 0}$ & $0.02^{* * *}$ & $0.03^{* * *}$ & $0.03^{* * *}$ & $0.02^{* *}$ & 0.00 & $0.01^{*}$ & $0.03^{* *}$ & $0.02^{* * *}$ & -0.01 \\
\hline & $\Theta_{\mathrm{k} 0}$ & $0.04^{* * *}$ & $0.03^{* * *}$ & 0.01 & 0.01 & $0.02^{* * *}$ & $0.03^{* * *}$ & 0.01 & $-0.02^{*}$ & $0.06^{* * *}$ \\
\hline \multirow{2}{*}{$\begin{array}{l}\text { Consumer } \\
\text { Confidence }\end{array}$} & $\mathrm{B}_{\mathrm{k} 0}$ & $0.02^{* * *}$ & $0.03^{* * *}$ & 0.00 & 0.00 & $0.01^{* *}$ & 0.01 & 0.00 & $0.01^{* * *}$ & 0.00 \\
\hline & $\Theta_{\mathrm{k} 0}$ & $0.03^{* * *}$ & $0.02^{* * *}$ & $0.03^{* *}$ & $0.04^{* *}$ & 0.00 & $0.04^{* * *}$ & 0.01 & -0.01 & 0.00 \\
\hline \multirow{2}{*}{ Retail Sales } & $\mathrm{B}_{\mathrm{k} 0}$ & $0.01^{*}$ & 0.01 & 0.01 & 0.02 & $0.03^{* * *}$ & $0.02^{* * *}$ & 0.00 & 0.00 & $-0.04^{*}$ \\
\hline & $\Theta_{\mathrm{k} 0}$ & $0.04^{* * *}$ & $0.04^{* * *}$ & 0.01 & $0.02^{* *}$ & $0.03^{* * *}$ & $0.02^{\star *}$ & $0.06^{* * *}$ & 0.00 & 0.04 \\
\hline Initial & $\mathrm{B}_{\mathrm{k} 0}$ & 0.00 & 0.00 & 0.01 & 0.00 & 0.00 & -0.01 & 0.00 & 0.00 & 0.00 \\
\hline Unemployment & $\Theta_{\mathrm{k} 0}$ & $0.02^{* * *}$ & $0.02^{* \star *}$ & 0.01 & $0.02^{\star \star *}$ & $0.01^{* * *}$ & $0.03^{* * *}$ & $0.01^{* *}$ & 0.00 & 0.01 \\
\hline
\end{tabular}

Notes: We estimate the exchange rate conditional mean model (7)

$R_{t}=\beta_{0}+\sum_{i=1}^{I} \beta_{i} R_{t-i}+\sum_{k=1}^{K} \sum_{j=0}^{J} \beta_{k j} S_{k, t-j}+\sum_{k=1}^{K} \sum_{j=0}^{J} \theta_{k j} D_{k, t-j}+\varepsilon_{t}$, where $\mathrm{D}_{\mathrm{k}, \mathrm{t}-\mathrm{j}}$ is dummy variable for the

announcement. We report estimates of the contemporaneous response of exchange-rate returns to news surprises, $\beta_{\mathrm{k} 0}$. We also estimate the disturbance volatility model (8) 
$\left|\hat{\varepsilon}_{t}\right|=c+\psi \frac{\hat{\sigma}_{d(t)}}{\sqrt{288}}+\sum_{k=1}^{K} \sum_{j^{\prime}=0}^{J^{\prime}}\left|S_{k, t-j^{\prime}}\right|+\sum_{k=1}^{K} \sum_{j^{\prime}=0}^{J^{\prime}} \theta_{k j^{\prime}} D_{k, t-j^{\prime}}+\left(\sum_{q=1}^{Q}\left(\delta_{q} \cos \left(\frac{q 2 \pi t}{288}\right)+\phi_{q} \sin \left(\frac{q 2 \pi t}{288}\right)\right)\right)+u_{t} \cdot$ Asterisks denote statistical significance (*** at 1 -percent level, $* *$ at 5 -percent level, and $*$ at 10 -percent level). 
Table 5. F-Test Results with Symmetric Response between Positive and Negative News Surprises

\begin{tabular}{|c|c|c|c|c|c|c|c|c|c|c|c|}
\hline News & & Euro & $\begin{array}{c}\text { Czech } \\
\text { Republic }\end{array}$ & Hungary & Indonesia & Korea & Mexico & Poland & $\begin{array}{l}\text { South } \\
\text { Africa }\end{array}$ & Thailand & Turkey \\
\hline \multirow{2}{*}{$\begin{array}{l}\text { Consumer } \\
\text { Confidence }\end{array}$} & Fvalue & 1.40 & 0.83 & 0.64 & 1.22 & 1.67 & 4.03 & 2.47 & 0.56 & 1.79 & 2.02 \\
\hline & Pvalue & 0.25 & 0.44 & 0.53 & 0.31 & 0.20 & 0.02 & 0.09 & 0.57 & 0.18 & 0.15 \\
\hline \multirow{2}{*}{$\begin{array}{l}\text { Durable Goods } \\
\text { Orders }\end{array}$} & Fvalue & 1.26 & 1.67 & 2.53 & 2.43 & 0.77 & 1.25 & 1.33 & 2.63 & 6.86 & 0.35 \\
\hline & Pvalue & 0.29 & 0.20 & 0.09 & 0.10 & 0.47 & 0.29 & 0.27 & 0.08 & 0.00 & 0.70 \\
\hline \multirow{2}{*}{$\begin{array}{l}\text { Gross Domestic } \\
\text { Product }\end{array}$} & Fvalue & 0.65 & 3.09 & 4.48 & 0.03 & 0.77 & 0.01 & 0.62 & 0.82 & 0.15 & 2.65 \\
\hline & Pvalue & 0.52 & 0.05 & 0.01 & 0.97 & 0.47 & 0.99 & 0.54 & 0.45 & 0.86 & 0.08 \\
\hline \multirow{2}{*}{$\begin{array}{l}\text { New Home } \\
\text { Sales }\end{array}$} & Fvalue & 2.31 & 0.23 & 0.02 & 0.22 & 0.43 & 0.55 & 1.02 & 0.20 & 1.78 & 0.88 \\
\hline & Pvalue & 0.11 & 0.80 & 0.98 & 0.81 & 0.65 & 0.58 & 0.37 & 0.82 & 0.18 & 0.42 \\
\hline \multirow{2}{*}{ Nonfarm Payroll } & Fvalue & 1.25 & 1.47 & 1.05 & 2.63 & 1.64 & 1.62 & 0.85 & 1.80 & 0.18 & 2.11 \\
\hline & Pvalue & 0.29 & 0.24 & 0.36 & 0.08 & 0.21 & 0.21 & 0.43 & 0.17 & 0.83 & 0.13 \\
\hline \multirow{2}{*}{ Producer Price } & Fvalue & 1.30 & 1.27 & 1.51 & 1.37 & 0.89 & 0.31 & 1.14 & 0.58 & 0.38 & 1.67 \\
\hline & Pvalue & 0.28 & 0.29 & 0.23 & 0.26 & 0.42 & 0.74 & 0.33 & 0.56 & 0.69 & 0.20 \\
\hline \multirow{2}{*}{ Retail Sales } & Fvalue & 0.03 & 0.93 & 0.66 & 0.34 & 0.29 & 2.14 & 2.81 & 0.34 & 1.56 & 0.70 \\
\hline & Pvalue & 0.97 & 0.40 & 0.52 & 0.71 & 0.75 & 0.13 & 0.07 & 0.71 & 0.22 & 0.50 \\
\hline \multirow{2}{*}{ Trade Balance } & Fvalue & 0.21 & 1.10 & 0.76 & 1.14 & 0.29 & 3.59 & 3.25 & 0.55 & 0.18 & 2.60 \\
\hline & Pvalue & 0.81 & 0.34 & 0.47 & 0.33 & 0.75 & 0.03 & 0.05 & 0.58 & 0.84 & 0.08 \\
\hline \multirow{2}{*}{$\begin{array}{l}\text { Initial } \\
\text { Unemployment }\end{array}$} & Fvalue & 1.60 & 4.10 & 3.70 & 0.06 & 3.32 & 0.54 & 2.41 & 1.10 & 1.00 & 1.83 \\
\hline & Pvalue & 0.20 & 0.02 & 0.03 & 0.94 & 0.04 & 0.58 & 0.09 & 0.33 & 0.37 & 0.16 \\
\hline
\end{tabular}

Notes: We estimate the exchange rate conditional mean model (9)

$R_{t}=\beta_{0 k} S_{k t}+\beta_{1 k} S_{k t}^{2}+D_{k t}\left(\beta_{2 k} S_{k t}+\beta_{3 k} S_{k t}^{2}\right)+\varepsilon_{t}$, where $D_{k, t}$ is dummy variable for that has value 1 if the new surprise is positive, and value of 0 if negative. The null hypothesis used in the test is $\beta_{2}=\beta_{3}=0$. 
Table 6. Impact of Major News Surprises with FX Forecasts

\begin{tabular}{|c|c|c|c|c|c|c|c|c|c|}
\hline \multicolumn{5}{|c|}{ Czech } & \multicolumn{5}{|c|}{ South } \\
\hline Announcements & Republic & Hungary & Indonesia & Korea & Mexico & Poland & Africa & Thailand & Turkey \\
\hline \multicolumn{10}{|c|}{ Impact of News Surprises Only } \\
\hline Durable Goods Orders & $0.05^{\star *}$ & $0.07^{* *}$ & $0.06^{* *}$ & $0.02^{*}$ & 0.01 & $0.06^{* *}$ & $0.06^{* *}$ & -0.02 & 0.04 \\
\hline Nonfarm Payroll & $0.19^{* *}$ & $0.21^{* *}$ & $0.07^{* *}$ & $0.08^{* *}$ & $0.11^{* *}$ & $0.23^{* *}$ & $0.26^{* *}$ & 0.01 & $0.14^{* *}$ \\
\hline Trade Balance & $0.04^{* *}$ & $0.06^{* *}$ & -0.01 & 0.01 & -0.01 & $0.11^{* *}$ & $0.09^{* *}$ & 0.01 & 0.03 \\
\hline Producer Price & -0.01 & -0.01 & $0.02^{*}$ & 0.01 & 0.01 & 0.01 & 0.03 & 0.00 & 0.02 \\
\hline New Home Sales & -0.02 & $0.03^{*}$ & $0.04^{* *}$ & $0.03^{* *}$ & 0.01 & $0.03^{* *}$ & -0.01 & -0.01 & -0.04 \\
\hline Gross Domestic Product & $0.09^{* *}$ & $0.09^{* *}$ & $0.06^{* *}$ & 0.02 & 0.01 & $0.07^{* \star}$ & $0.09^{* *}$ & 0.00 & 0.00 \\
\hline Consumer confidence & $0.12^{\star \star}$ & $0.10^{\star *}$ & 0.02 & 0.02 & 0.02 & $0.07^{\star *}$ & $0.05^{\star *}$ & 0.00 & 0.10 \\
\hline Retail Sales & $0.09^{* *}$ & $0.07^{* *}$ & $0.04^{* *}$ & $0.04^{* *}$ & 0.02 & $0.08^{* *}$ & $0.03^{*}$ & 0.01 & -0.01 \\
\hline Initial Unemployment & $-0.04^{* *}$ & $-0.03^{* *}$ & -0.01 & $-0.02^{* *}$ & $-0.02^{* *}$ & $-0.02^{* *}$ & $-0.02^{* *}$ & 0.01 & 0.01 \\
\hline \multicolumn{10}{|c|}{ Impact of News Surprises with FX Forecasts } \\
\hline Durable Goods Orders & $0.22^{* *}$ & $0.19^{* *}$ & $1.08^{*}$ & 0.39 & $0.04^{* *}$ & 0.10 & 0.29 & -1.04 & $0.00^{*}$ \\
\hline Nonfarm Payroll & $0.73^{* *}$ & $0.65^{\star *}$ & $1.36^{* *}$ & $1.07^{*}$ & $0.10^{* *}$ & $-0.52^{*}$ & -0.13 & $-1.80^{* *}$ & $0.00^{* *}$ \\
\hline Trade Balance & $0.38^{* *}$ & $0.31^{* *}$ & -0.74 & $1.12^{*}$ & -0.01 & $-0.84^{*}$ & $-1.00^{* *}$ & 0.06 & 0.00 \\
\hline Producer Price & $0.13^{* *}$ & $0.18^{* *}$ & 0.50 & 0.16 & 0.01 & 0.24 & 0.11 & 0.43 & $0.00^{*}$ \\
\hline New Home Sales & $0.18^{* *}$ & -0.01 & $0.89^{*}$ & -0.75 & 0.01 & 0.00 & 0.34 & 0.28 & 0.00 \\
\hline Gross Domestic Product & $0.20^{* *}$ & $0.24^{* *}$ & $0.87^{*}$ & $0.81^{*}$ & 0.03 & 0.17 & -0.51 & -0.18 & 0.00 \\
\hline Consumer confidence & $-0.11^{*}$ & -0.03 & 0.52 & $2.35^{* *}$ & $0.05^{* *}$ & $-1.02^{* *}$ & 0.15 & 0.65 & 0.00 \\
\hline Retail Sales & 0.06 & $0.13^{*}$ & $1.85^{\star *}$ & -0.29 & $0.12^{* *}$ & 0.30 & 0.39 & -0.49 & 0.00 \\
\hline Initial Unemployment & -0.01 & -0.04 & -0.09 & -0.16 & $-0.03^{\star *}$ & $0.44^{* *}$ & 0.14 & 0.32 & 0.00 \\
\hline
\end{tabular}

Notes: We estimate the exchange rate conditional mean model (10)

$R_{t}=\beta_{0}+\sum_{i=1}^{I} \beta_{i} R_{t-i}+\sum_{k=1}^{K} \sum_{j=0}^{J} \beta_{k j} S_{k, t-j}+\sum_{k=1}^{K} \sum_{j=0}^{J} \gamma_{k j} F X D_{d, t-j} S_{k, t-j}+\varepsilon_{t}$, where $F X D_{d, t}$ is the index that measures the change between consensus and spot price. Asterisks denote statistical significance (*** at 1percent level, ** at 5-percent level, and * at 10-percent level). 
Table 7. Impact of Major News Surprises with FX Forecasts Dispersion

\begin{tabular}{|c|c|c|c|c|c|c|c|c|c|}
\hline \multicolumn{5}{|c|}{ Czech } & \multicolumn{5}{|c|}{ South } \\
\hline Announcements & Republic & Hungary & ndonesia & Korea & Mexico & Poland & Africa & Thailand & Turkey \\
\hline \multicolumn{10}{|c|}{ Impact of News Surprises Only } \\
\hline Consumer Confidence & $0.08^{\star *}$ & $0.09^{* *}$ & -0.01 & 0.03 & -0.03 & $0.07^{* *}$ & 0.04 & -0.01 & 0.12 \\
\hline Durable Goods Order & $0.12^{* *}$ & $0.11^{* *}$ & $0.13^{* *}$ & 0.02 & 0.01 & $0.06^{* *}$ & 0.03 & -0.05 & $0.12^{*}$ \\
\hline Gross Domestic Product & t $0.19^{* *}$ & $0.09^{* *}$ & $0.11^{* *}$ & 0.01 & 0.01 & 0.04 & 0.03 & 0.04 & 0.01 \\
\hline New Home Sales & 0.00 & $0.07^{* *}$ & -0.02 & 0.01 & -0.02 & 0.01 & -0.05 & -0.05 & -0.05 \\
\hline Nonfarm Payroll & $0.25^{* *}$ & $0.17^{\star *}$ & $0.08^{* *}$ & $0.19^{* *}$ & $0.10^{* *}$ & $0.47^{* *}$ & $0.16^{* *}$ & 0.00 & $0.17^{* *}$ \\
\hline Producer Price & 0.02 & -0.01 & $0.04^{*}$ & $0.05^{*}$ & -0.01 & 0.02 & -0.01 & 0.00 & $0.12^{* *}$ \\
\hline Retail Sales & $0.16^{* *}$ & $0.06^{* *}$ & $0.07^{* *}$ & 0.02 & $0.14^{* *}$ & $0.14^{* *}$ & 0.03 & 0.02 & -0.03 \\
\hline Trade Balance & $0.07^{* *}$ & $0.17^{\star *}$ & -0.03 & 0.02 & -0.04 & $0.10^{* *}$ & 0.02 & -0.02 & 0.03 \\
\hline Initial Jobless Claim & $-0.05^{* *}$ & $-0.03^{* *}$ & -0.01 & 0.01 & -0.02 & $-0.02^{*}$ & -0.01 & 0.01 & 0.01 \\
\hline \multicolumn{10}{|c|}{ Impact of News Surprises with Dispersions } \\
\hline Consumer Confidence & $0.61^{*}$ & 0.32 & 0.10 & -0.09 & 0.04 & -0.20 & 0.08 & 0.16 & -0.31 \\
\hline Durable Goods Order & $-0.76^{* *}$ & -0.54 & $-0.60^{* *}$ & 0.16 & -0.47 & -0.02 & 0.15 & 0.34 & $-0.82^{* *}$ \\
\hline Gross Domestic Product & $t-1.96^{* *}$ & 0.58 & -0.51 & 0.15 & -0.31 & 0.33 & 0.26 & -0.72 & 0.06 \\
\hline New Home Sales & 0.11 & $-0.75^{*}$ & 0.43 & 0.28 & 0.38 & 0.18 & 0.28 & 0.55 & 0.20 \\
\hline Nonfarm Payroll & $0.87^{* *}$ & $2.23^{* *}$ & -0.21 & $-1.59^{* *}$ & $-1.36^{\star *}$ & $-2.02^{* *}$ & $0.50^{*}$ & 0.05 & $-0.76^{* *}$ \\
\hline Producer Price & -0.16 & $0.50^{*}$ & -0.23 & -0.57 & 0.35 & -0.02 & 0.22 & 0.12 & $-0.68^{* *}$ \\
\hline Retail Sales & $-0.98^{* *}$ & 0.45 & -0.28 & 0.25 & $-3.42^{* *}$ & $-0.45^{*}$ & 0.03 & -0.17 & 0.06 \\
\hline Trade Balance & $0.83^{* *}$ & $-1.10^{* *}$ & 0.31 & 0.03 & 0.49 & 0.05 & 0.34 & 0.37 & -0.10 \\
\hline Initial Jobless Claim & 0.17 & -0.03 & 0.03 & $-0.47^{*}$ & 0.33 & 0.00 & -0.07 & -0.04 & 0.00 \\
\hline
\end{tabular}

Notes: We estimate the exchange rate conditional mean model (12)

$R_{t}=\beta_{0}+\sum_{i=1}^{I} \beta_{i} R_{t-i}+\sum_{k=1}^{K} \sum_{j=0}^{J} \beta_{k j} S_{k, t-j}+\sum_{k=1}^{K} \gamma_{k} D I S P_{d, t} S_{k, t}+\varepsilon_{t}$, where DISP is the index that measures the magnitude of dispersions between consensus and spot price, defined by

$D I S P_{d, t}=\left|\frac{C F X_{d, t}^{\text {high }}-C F X_{d, t}^{\text {low }}}{S F X_{t}}\right|, C F X_{d, t}^{\text {high }}$ means the maximum of FX forecasts at time $t$, and

$C F X_{d, t}^{\text {low }}$ means the minimum of FX forecasts at time $t$. Asterisks denote statistical significance (*** at 1percent level, ${ }^{* *}$ at 5-percent level, and $*$ at 10-percent level). 
Figure 1. Sample Autocorrelation Graphs of 5-minute Returns across Countries
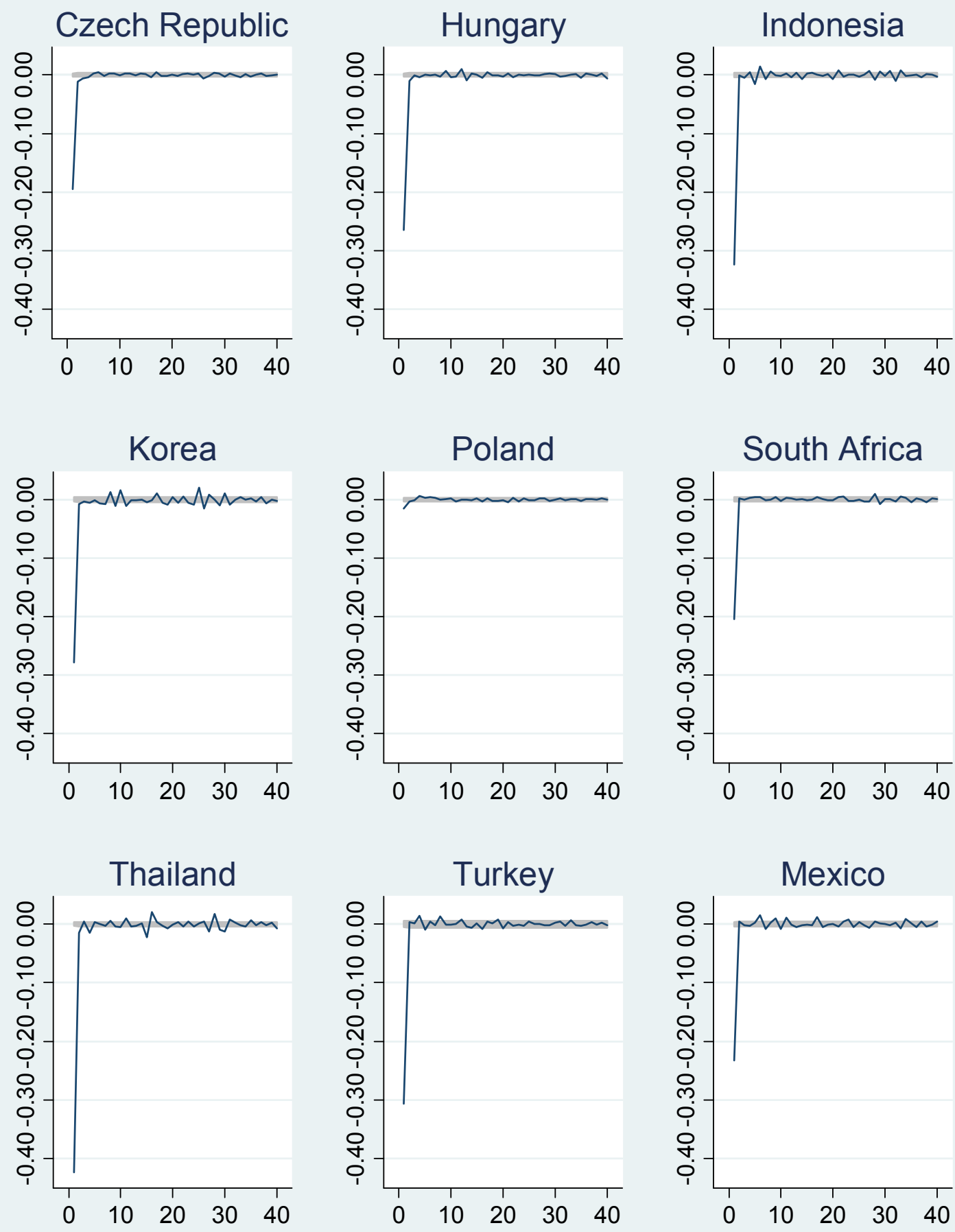

Note: Shaded area in each graph represents Bartlett's formula for MA(q) $95 \%$ confidence bands under the null hypothesis of white noise. 
Figure 2. Sample Autocorrelation Graphs of 5-minute Absolute Returns across Countries
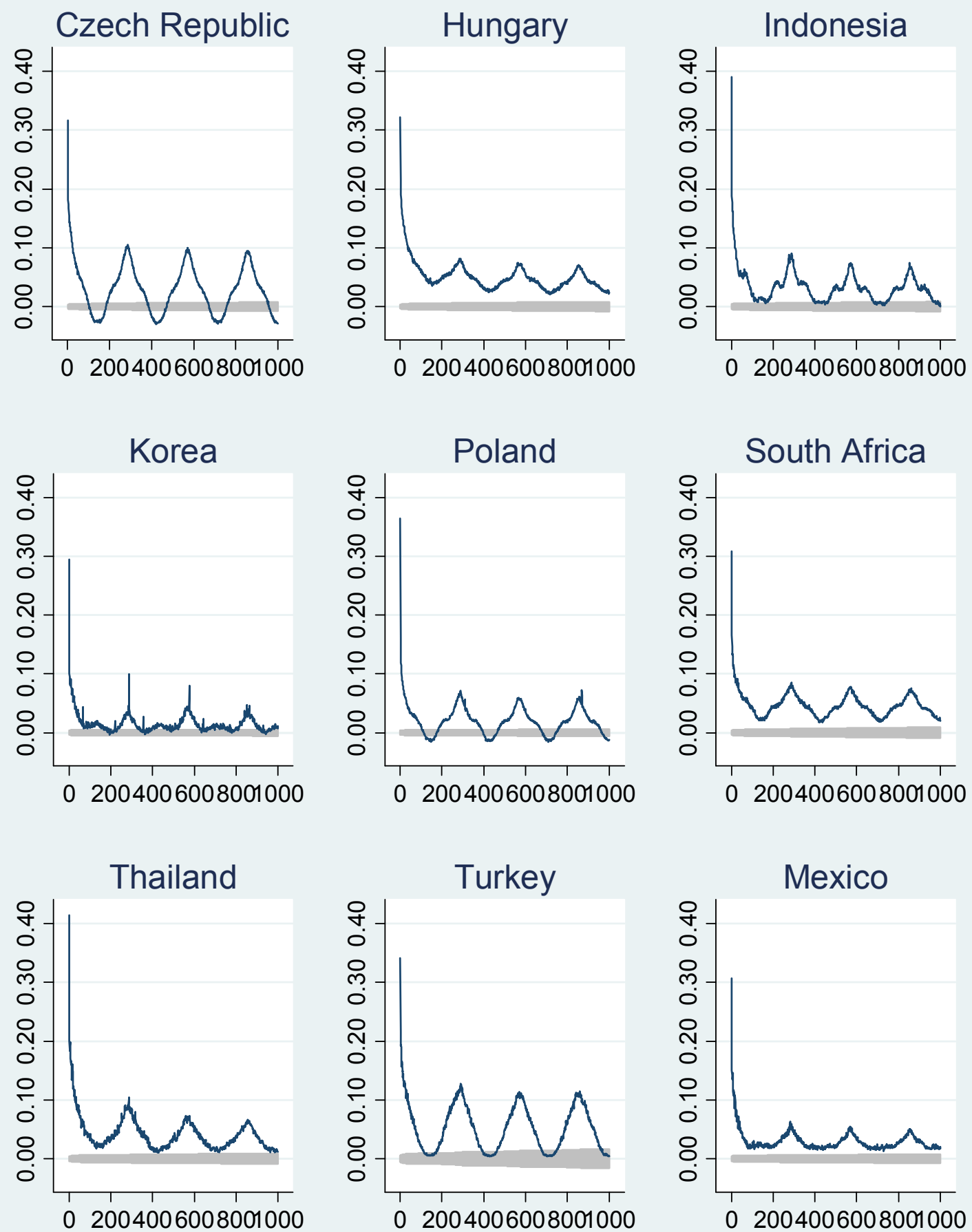

Note: Shaded area in each graph represents Bartlett's formula for MA(q) $95 \%$ confidence bands under the null hypothesis of white noise. 
Figure 3: Evolution of EM Exchange Rates Responses to U.S. News

We estimate equation (3) using a two-year moving window. In the following charts, bars indicate the significant point estimates from the rolling regressions. Insignificant estimates are left blank. The lines show the number of observations in each regression. As the whole sample covers $2000-2007$, and the window is for two years, these statistics end at 2005 .
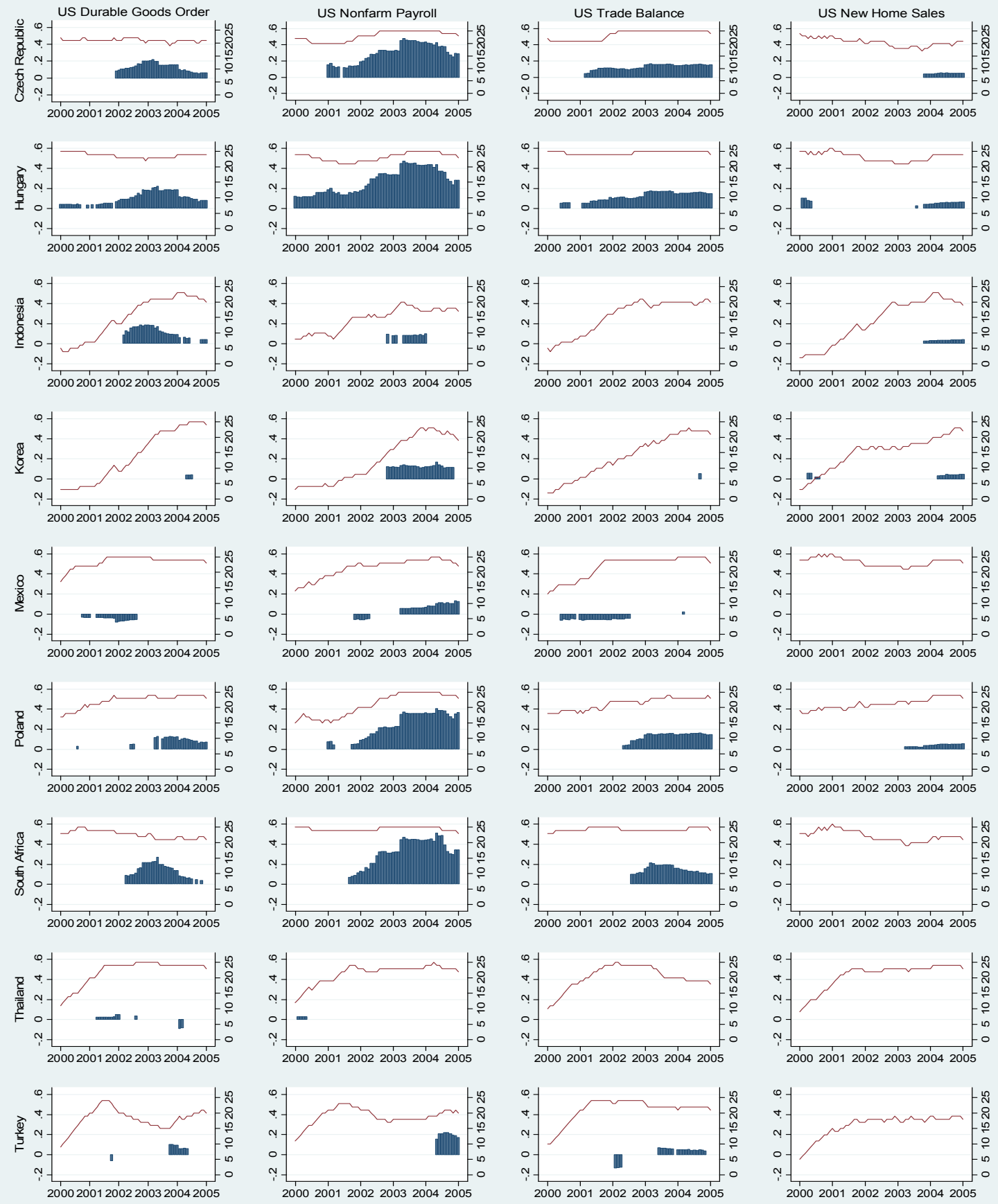
Figure 3: Evolution of EM Exchange Rates Responses to U.S. News (continued)
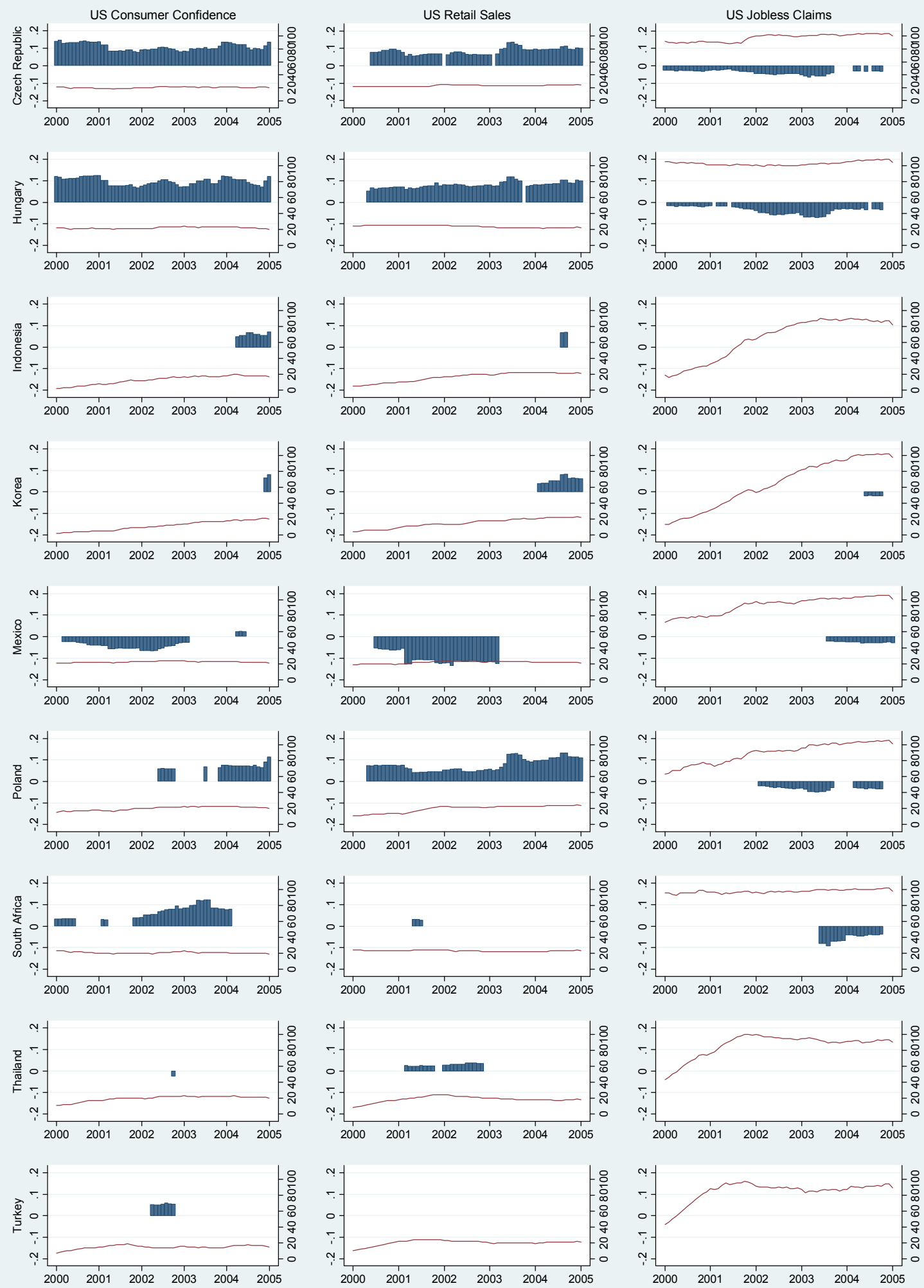
Figure 4. U.S. News Impact Curve

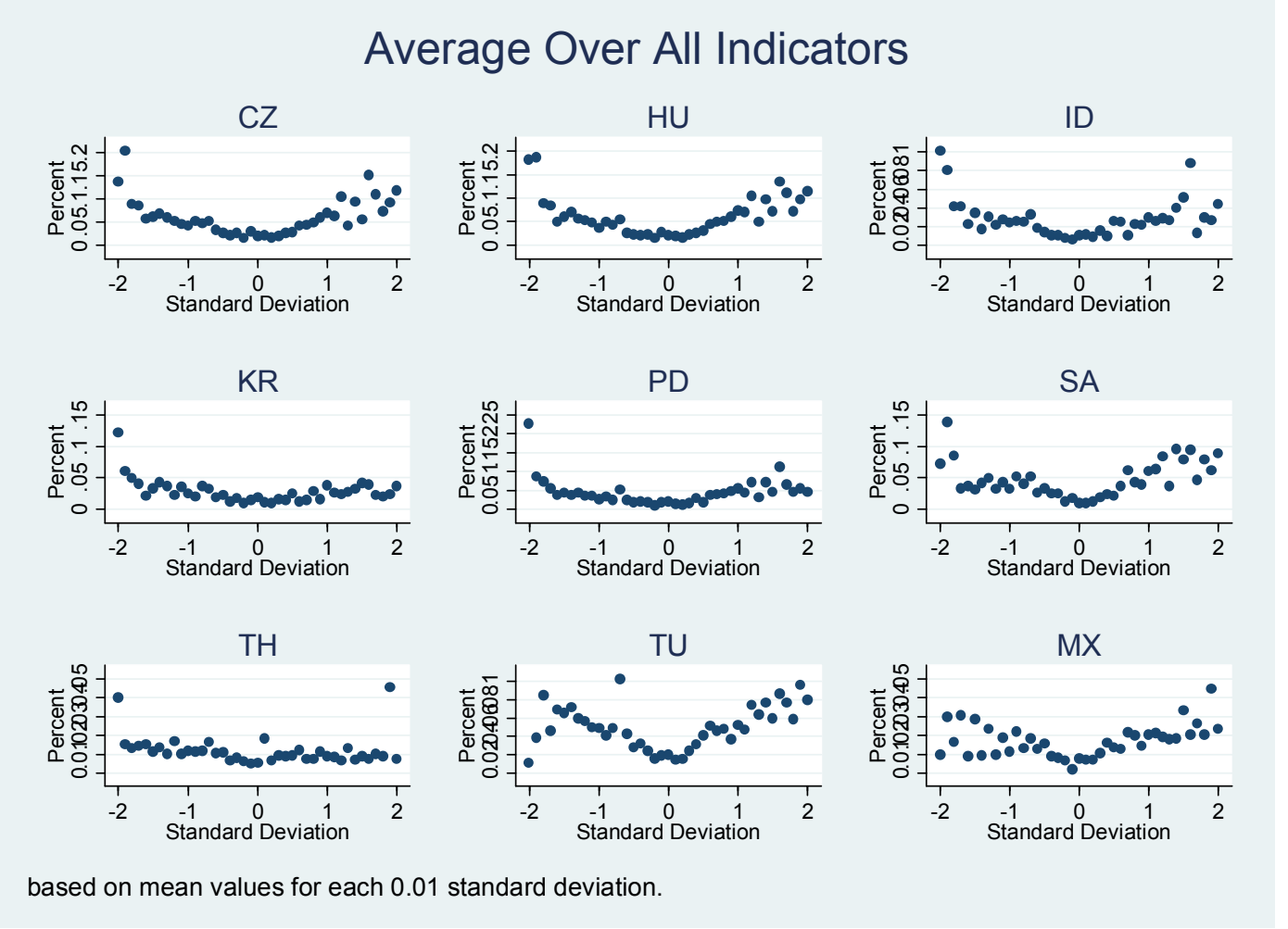




\section{Appendix}

Appendix 1. Summary Table for FX Time Series

\begin{tabular}{lccc}
\hline \hline \multicolumn{1}{c}{ Country } & $\begin{array}{c}\text { Number of } \\
\text { Observations }\end{array}$ & $\begin{array}{c}\text { Number of Nonmissing } \\
\text { Observation }\end{array}$ & Period \\
\hline Czech Republic & 736,416 & 479,119 & January 2, 2000 December 31, 2006 \\
Hungary & 736,416 & 517,950 & January 2, 2000 December 31, 2006 \\
Indonesia & 736,416 & 365,843 & January 2, 2000 December 31, 2006 \\
Korea & 727,488 & 341,508 & January 2, 2000 D December 31, 2006 \\
Mexico & 736,416 & 302,674 & January 2, 2000 December 31, 2006 \\
Poland & 736,416 & 409,279 & January 2, 2000 D December 31, 2006 \\
South Africa & 736,416 & 366,973 & January 2, 2000 December 31, 2006 \\
Thailand & 736,416 & 446,514 & January 2, 2000 D December 31, 2006 \\
Turkey & 631,008 & 175,967 & January 2, 2001 December 31, 2006 \\
\hline \hline
\end{tabular}

Source: Olsen Financial Technology (www.olsendata.com)

* For Korea, January 2004 data is not included. 
Appendix 2. Exchange Regime Changes from 2000 to 2006

\begin{tabular}{|c|c|c|c|c|}
\hline Country & Currency & Period & Classification & Notes \\
\hline $\begin{array}{l}\text { Czech } \\
\text { Republic }\end{array}$ & Czech koruna & full sample & $\begin{array}{l}\text { Managed floating with } \\
\text { no predetermined path } \\
\text { for the exchange rate }\end{array}$ & $\begin{array}{l}\text { The external value of the koruna is determined by supply and demand in the foreign exchange } \\
\text { market. The Czech National Bank (CNB) may intervene in the foreign exchange market in order } \\
\text { to smooth large intraday volatility swings of the Euro-koruna rate. The CNB publishes daily rates } \\
\text { of } 29 \text { selected currencies against the koruna for customs and accounting purposes. Commercial } \\
\text { banks set their own exchange rate with no limitation. }\end{array}$ \\
\hline \multirow[t]{6}{*}{ Hungary } & Hungarian forint & 6/4/2003 -- current & $\begin{array}{l}\text { Pegged exchange rate } \\
\text { within horizontal bands }\end{array}$ & $\begin{array}{l}\text { The Hungarian forint trades against the Euro within a band of } \pm 15 \% \text { around the central parity, } \\
\text { which is fixed to the Euro at Ft } 282.36 \text { per } € 1 \text {. }\end{array}$ \\
\hline & & $10 / 1 / 2001--6 / 4 / 2004$ & $\begin{array}{l}\text { Pegged exchange rate } \\
\text { within horizontal bands }\end{array}$ & $\begin{array}{l}\text { The crawling peg was abolished and the central parity of the forint was fixed to the Euro at Ft } \\
276.1 \text { per } € 1 \text {. Thus, the exchange arrangement of the forint was reclassified to the category } \\
\text { pegged exchange rate within horizontal bands from the category crawling band. (10/1/2001) }\end{array}$ \\
\hline & & $5 / 4 / 2001-10 / 1 / 2001$ & Crawling Band & $\begin{array}{l}\text { The width of the band within which the forint trades against the Euro was widened to } \pm 15 \% \text { from } \\
\pm 2.25 \% \text { around the parity. }(5 / 4 / 2001)\end{array}$ \\
\hline & & $4 / 1 / 2001--5 / 4 / 2001$ & Crawling Band & The monthly depreciation of the forint was adjusted to $0.2 \%$ from $0.3 \%(4 / 1 / 2001)$ \\
\hline & & $4 / 1 / 2000--4 / 1 / 2001$ & Crawling Band & The monthly depreciation of the forint was adjusted to $0.3 \%$ from $0.4 \%(4 / 1 / 2000)$ \\
\hline & & $1 / 1 / 2000--4 / 1 / 2000$ & Crawling Band & The preannounced rate of crawl against the Euro was affected. $(1 / 1 / 2000)$ \\
\hline Indonesia & Indonesian rupiah & full sample & $\begin{array}{l}\text { Managed floating with } \\
\text { no predetermined path } \\
\text { for the exchange rate }\end{array}$ & $\begin{array}{l}\text { The exchange rate is determined by supply and demand conditions in the foreign exchange } \\
\text { market. However, the Bank Indonesia (BI) may intervene in the foreign exchange market to } \\
\text { maintain stability of the exchange rate. }\end{array}$ \\
\hline Korea & Korean won & full sample & Independently floating & $\begin{array}{l}\text { The exchange rate of the won is determined on the basis of supply and demand in the foreign } \\
\text { exchange market. However, the authorities intervene when necessary to counter disorderly } \\
\text { conditions in the market. }\end{array}$ \\
\hline
\end{tabular}


The exchange rate of the peso is determined freely in the foreign exchange market. The Exchange Commission established a rules-based mechanism to reduce the rate of international reserves accumulation. The Bank of Mexico (BOM) sells dollars directly in the foreign exchange market every day according to the following procedure: the BOM announces every quarter the total amount of dollars it will offer to the currency market each day during the following four quarters. The total amount of dollars to be sold will equal $50 \%$ of the net international reserves accumulated during the previous quarter, with one-fourth of the established amount being auctioned each quarter, not including the cumulative amount of dollars sold through the auction mechanism during the same period. Based on the total amount of dollars, the BOM auctions on a daily basis a fixed amount of dollars following a preestablished schedule (the daily amount to be

Mexico Mexican peso full sample Independently floating sold is determined according to the number of working days in the current quarter).

\section{Poland} Polish zloty $4 / 12 / 2000$-- current

Independently floating

The exchange rate of the zloty is determined on the basis of supply and demand in the foreign exchange market, and the zloty is traded freely against all currencies.

3/24/1999 -$4 / 12 / 2000$ Crawling peg

Effective 1/1/1999, the currency basket was changed to 55\% Euro and 45\% dollar. Effective $3 / 24 / 1999$, the width of the band was increased to $\pm 15 \%$ around the central parity.

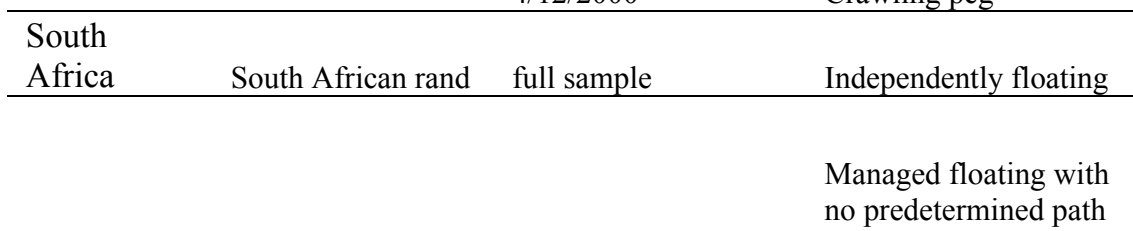
market.
The exchange rate of the rand is determined by demand and supply in the foreign exchange no predetermined path

The exchange rate of the baht is determined in the foreign exchange market. The baht-dollar reference exchange rate is announced daily, based on the average exchange rate of the previous day. The authorities intervene in the foreign exchange market as conditions require. 
New Turkish lira

(YTL $1=$ TL 1

Turkey

\section{million, 1/1/2006)}

The lira was allowed to float. As a consequence, the exchange rate arrangement was reclassified to the category independently floating from the category crawling peg $(2 / 22 / 2001)$. The exchange rate of the lira is determined on the basis of supply and demand in the foreign exchange market. The Central Bank of the Republic of Turkey (CBRT) conducts daily auctions to build up reserves hereby it buys a fixed amount of dollars and provides the successful bidders with the option to purchase up to $200 \%$ of their successful bid amount at the average auction price. The daily fixed purchase amount was raised to $\$ 20$ million in 2006 . The daily foreign exchange purchase

auctions were suspended on May 16, 2006, in response to financial market volatility. On June 26 and June 27, 2006, the CBRT held foreign exchange auctions under which it sold \$500 million on each day through multiple price auctions. On November 10, 2006, the CRBT resumed its daily foreign exchange auction program, with a daily fixed purchase amount of $\$ 15$ million. The CBRT reserves the right to intervene in the foreign exchange market in case of excessive volatility in the foreign exchange rates.

In December 1999, the Central Bank of Turkey (CBT) modified its exchange arrangement by moving to a preannouncement of the exchange rate path of the lira against the current basket comprising the dollar and the Euro (in amounts equivalent to $\$ 1$ and $€ 0.77$ ). 
Appendix 3a. Summary Statistics for Market Forecast

\begin{tabular}{|c|c|c|c|c|c|c|}
\hline Country & \# month ahead & Mean & Median & Max & Min & Std. Dev. \\
\hline \multirow{4}{*}{ Czech Republic } & 1 & 0.004 & 0.006 & 0.024 & -0.015 & 0.008 \\
\hline & 3 & 0.004 & 0.005 & 0.035 & -0.021 & 0.010 \\
\hline & 12 & -0.002 & -0.005 & 0.092 & -0.040 & 0.022 \\
\hline & 24 & -0.004 & -0.007 & 0.053 & -0.052 & 0.026 \\
\hline \multirow{4}{*}{ Hungary } & 1 & 0.005 & 0.006 & 0.034 & -0.037 & 0.013 \\
\hline & 3 & 0.008 & 0.010 & 0.045 & -0.034 & 0.017 \\
\hline & 12 & 0.016 & 0.013 & 0.077 & -0.040 & 0.030 \\
\hline & 24 & 0.013 & 0.016 & 0.081 & -0.070 & 0.034 \\
\hline \multirow{4}{*}{ Indonesia } & 1 & 0.002 & -0.001 & 0.140 & -0.043 & 0.023 \\
\hline & 3 & 0.000 & -0.001 & 0.172 & -0.048 & 0.029 \\
\hline & 12 & -0.005 & -0.002 & 0.169 & -0.071 & 0.038 \\
\hline & 24 & -0.008 & -0.007 & 0.133 & -0.111 & 0.040 \\
\hline \multirow{4}{*}{ Korea } & 1 & 0.000 & 0.000 & 0.032 & -0.034 & 0.013 \\
\hline & 3 & -0.004 & -0.004 & 0.034 & -0.047 & 0.016 \\
\hline & 12 & -0.024 & -0.023 & 0.016 & -0.067 & 0.018 \\
\hline & 24 & -0.030 & -0.028 & 0.019 & -0.084 & 0.023 \\
\hline \multirow{4}{*}{ Mexico } & 1 & 0.006 & 0.006 & 0.032 & -0.028 & 0.014 \\
\hline & 3 & 0.015 & 0.017 & 0.054 & -0.023 & 0.019 \\
\hline & 12 & 0.046 & 0.045 & 0.108 & -0.011 & 0.029 \\
\hline & 24 & 0.076 & 0.073 & 0.176 & 0.020 & 0.035 \\
\hline \multirow{4}{*}{ Poland } & 1 & -0.001 & -0.003 & 0.056 & -0.046 & 0.022 \\
\hline & 3 & -0.005 & -0.007 & 0.048 & -0.058 & 0.026 \\
\hline & 12 & -0.015 & -0.017 & 0.071 & -0.097 & 0.042 \\
\hline & 24 & 0.005 & 0.001 & 0.115 & -0.104 & 0.052 \\
\hline \multirow{4}{*}{ South Africa } & 1 & 0.009 & 0.009 & 0.082 & -0.051 & 0.030 \\
\hline & 3 & 0.020 & 0.016 & 0.119 & -0.056 & 0.041 \\
\hline & 12 & 0.060 & 0.063 & 0.216 & -0.061 & 0.069 \\
\hline & 24 & 0.110 & 0.111 & 0.319 & -0.043 & 0.096 \\
\hline \multirow{4}{*}{ Thailand } & 1 & 0.000 & 0.003 & 0.024 & -0.035 & 0.012 \\
\hline & 3 & -0.003 & -0.001 & 0.022 & -0.045 & 0.016 \\
\hline & 12 & -0.014 & -0.013 & 0.030 & -0.056 & 0.020 \\
\hline & 24 & -0.022 & -0.023 & 0.024 & -0.066 & 0.022 \\
\hline \multirow{4}{*}{ Turkey } & 1 & 0.024 & 0.023 & 0.097 & -0.066 & 0.033 \\
\hline & 3 & 0.057 & 0.049 & 0.165 & -0.057 & 0.048 \\
\hline & 12 & 0.164 & 0.146 & 0.378 & -0.040 & 0.107 \\
\hline & 24 & 0.298 & 0.305 & 0.652 & -0.013 & 0.186 \\
\hline
\end{tabular}


Appendix 3b. Summary Statistics for Market Forecast Dispersion

\begin{tabular}{|c|c|c|c|c|c|c|}
\hline Country & \# month ahead & Mean & Median & Max & Min & Std. Dev. \\
\hline \multirow{4}{*}{ Czech Republic } & 1 & 0.049 & 0.042 & 0.140 & 0.008 & 0.029 \\
\hline & 3 & 0.061 & 0.055 & 0.210 & 0.026 & 0.027 \\
\hline & 12 & 0.122 & 0.104 & 0.385 & 0.043 & 0.057 \\
\hline & 24 & 0.179 & 0.157 & 0.395 & 0.053 & 0.071 \\
\hline \multirow{4}{*}{ Hungary } & 1 & 0.058 & 0.055 & 0.132 & 0.007 & 0.031 \\
\hline & 3 & 0.067 & 0.061 & 0.145 & 0.009 & 0.030 \\
\hline & 12 & 0.120 & 0.107 & 0.230 & 0.047 & 0.043 \\
\hline & 24 & 0.144 & 0.150 & 0.224 & 0.054 & 0.039 \\
\hline \multirow{4}{*}{ Indonesia } & 1 & 0.121 & 0.096 & 0.468 & 0.045 & 0.085 \\
\hline & 3 & 0.158 & 0.142 & 0.518 & 0.053 & 0.083 \\
\hline & 12 & 0.269 & 0.259 & 0.540 & 0.119 & 0.108 \\
\hline & 24 & 0.346 & 0.344 & 0.667 & 0.154 & 0.121 \\
\hline \multirow{4}{*}{ Mexico } & 1 & 0.063 & 0.063 & 0.116 & 0.022 & 0.020 \\
\hline & 3 & 0.080 & 0.079 & 0.174 & 0.038 & 0.025 \\
\hline & 12 & 0.103 & 0.101 & 0.168 & 0.052 & 0.024 \\
\hline & 24 & 0.110 & 0.111 & 0.200 & 0.030 & 0.041 \\
\hline \multirow{4}{*}{ Poland } & 1 & 0.110 & 0.098 & 0.342 & 0.033 & 0.053 \\
\hline & 3 & 0.132 & 0.136 & 0.242 & 0.053 & 0.036 \\
\hline & 12 & 0.202 & 0.200 & 0.337 & 0.101 & 0.052 \\
\hline & 24 & 0.228 & 0.216 & 0.416 & 0.124 & 0.071 \\
\hline \multirow{4}{*}{ South Africa } & 1 & 0.177 & 0.171 & 0.506 & 0.058 & 0.074 \\
\hline & 3 & 0.226 & 0.210 & 0.575 & 0.081 & 0.089 \\
\hline & 12 & 0.315 & 0.303 & 0.807 & 0.135 & 0.111 \\
\hline & 24 & 0.347 & 0.323 & 0.664 & 0.165 & 0.120 \\
\hline \multirow{4}{*}{ Korea } & 1 & 0.071 & 0.065 & 0.183 & 0.032 & 0.029 \\
\hline & 3 & 0.103 & 0.100 & 0.220 & 0.047 & 0.026 \\
\hline & 12 & 0.175 & 0.178 & 0.252 & 0.104 & 0.035 \\
\hline & 24 & 0.209 & 0.199 & 0.324 & 0.133 & 0.047 \\
\hline \multirow{4}{*}{ Thailand } & 1 & 0.063 & 0.060 & 0.206 & 0.023 & 0.025 \\
\hline & 3 & 0.089 & 0.085 & 0.205 & 0.046 & 0.031 \\
\hline & 12 & 0.130 & 0.127 & 0.226 & 0.073 & 0.031 \\
\hline & 24 & 0.150 & 0.145 & 0.369 & 0.072 & 0.050 \\
\hline \multirow{4}{*}{ Turkey } & 1 & 0.178 & 0.146 & 0.449 & 0.071 & 0.093 \\
\hline & 3 & 0.227 & 0.194 & 0.512 & 0.095 & 0.111 \\
\hline & 12 & 0.382 & 0.334 & 0.817 & 0.161 & 0.170 \\
\hline & 24 & 0.605 & 0.588 & 1.320 & 0.187 & 0.283 \\
\hline
\end{tabular}


Appendix 4. Return and Volatility News Response Coefficients

\begin{tabular}{|c|c|c|c|c|c|c|c|c|c|}
\hline \multicolumn{5}{|c|}{ Czech } & \multicolumn{5}{|c|}{ South } \\
\hline Announcements & Republic & Hungary I & ndonesia & Korea & Mexico & Poland & Africa & Thailand & Turkey \\
\hline \multicolumn{10}{|c|}{ Impact of News Surprises on FX Returns } \\
\hline Business Inventories & 0.00 & -0.01 & 0.00 & -0.01 & $0.01^{*}$ & $-0.02^{* *}$ & $-0.03^{*}$ & 0.00 & $-0.04^{* *}$ \\
\hline Budget Deficit & 0.00 & 0.01 & 0.01 & $0.14^{* *}$ & 0.00 & 0.00 & 0.05 & 0.01 & 0.00 \\
\hline Current Account & $0.07^{* *}$ & $0.05^{* *}$ & $-0.08^{* *}$ & 0.01 & -0.01 & 0.01 & 0.05 & $0.03^{*}$ & 0.06 \\
\hline Capital Utilization & 0.00 & 0.00 & -0.02 & $-0.02^{\star *}$ & -0.01 & 0.01 & $0.13^{* *}$ & 0.01 & 0.04 \\
\hline Consumer Confidence & $0.11^{* *}$ & $0.10^{* *}$ & 0.01 & $0.02^{\star \star}$ & $-0.03^{* *}$ & $0.04^{* *}$ & $0.05^{\star *}$ & 0.00 & 0.05 \\
\hline Consumer Credit & -0.01 & -0.01 & -0.01 & -0.01 & 0.00 & 0.00 & -0.02 & 0.00 & 0.00 \\
\hline Construction Spending & $0.03^{* *}$ & $0.02^{* *}$ & 0.02 & 0.00 & 0.00 & 0.01 & -0.02 & $-0.04^{* *}$ & 0.00 \\
\hline Consumer Price Index & 0.00 & $0.02^{* *}$ & $-0.04^{* *}$ & 0.00 & $0.02^{* *}$ & 0.00 & $0.04^{* *}$ & 0.00 & $0.08^{* *}$ \\
\hline Durable Goods Orders & $0.05^{\star *}$ & $0.06^{* *}$ & $0.04^{\star *}$ & $0.03^{*}$ & $-0.01^{*}$ & $0.04^{* *}$ & $0.05^{* *}$ & $-0.03^{\star *}$ & -0.01 \\
\hline Factory Orders & $0.02^{* *}$ & $0.02^{* *}$ & 0.00 & -0.01 & 0.00 & 0.00 & $0.03^{*}$ & 0.00 & $0.08^{* *}$ \\
\hline Gross Domestic Product & $0.10^{* *}$ & $0.11^{* *}$ & $0.07^{* *}$ & $0.02^{* *}$ & 0.00 & $0.07^{* *}$ & $0.07^{* *}$ & -0.01 & 0.03 \\
\hline Housing Starts & $0.01^{*}$ & 0.01 & -0.01 & $0.01^{*}$ & -0.01 & 0.01 & 0.02 & -0.01 & 0.03 \\
\hline Imports & 0.00 & -0.01 & $0.02^{*}$ & $0.03^{* *}$ & $0.05^{* *}$ & $-0.01^{*}$ & -0.02 & -0.01 & $-0.05^{\star *}$ \\
\hline Interest rate & 0.01 & -0.01 & 0.00 & 0.00 & 0.01 & 0.00 & 0.00 & 0.01 & 0.00 \\
\hline Industrial production & $0.02^{* *}$ & $0.02^{*}$ & $0.03^{* *}$ & 0.01 & 0.00 & 0.00 & -0.01 & -0.01 & -0.02 \\
\hline NAPM & $1.36^{* *}$ & $1.27^{* *}$ & 0.07 & $0.34^{*}$ & 0.00 & $0.65^{\star *}$ & 0.31 & -0.27 & 0.00 \\
\hline Leading Indicators & 0.00 & $0.02^{*}$ & -0.01 & -0.01 & -0.01 & 0.00 & -0.01 & 0.01 & 0.00 \\
\hline New Home Sales & $0.02^{* *}$ & $0.03^{* *}$ & $0.03^{* *}$ & $0.03^{* *}$ & $0.01^{*}$ & $0.03^{* *}$ & 0.00 & -0.01 & -0.02 \\
\hline Nonfarm Payroll & $0.19^{* *}$ & $0.21^{* *}$ & $0.04^{* *}$ & $0.09^{* *}$ & $0.02^{* *}$ & $0.18^{* *}$ & $0.18^{* *}$ & 0.00 & $0.02^{*}$ \\
\hline Personal Spending & 0.00 & 0.01 & 0.01 & 0.00 & 0.00 & -0.01 & 0.01 & -0.01 & 0.00 \\
\hline Personal Income & 0.00 & 0.00 & -0.01 & 0.00 & 0.01 & 0.00 & -0.01 & 0.00 & 0.02 \\
\hline Producer Price & $0.02^{* *}$ & $0.02^{* *}$ & $0.04^{* *}$ & 0.01 & $0.01^{*}$ & $0.02^{* *}$ & $0.05^{* *}$ & 0.00 & -0.01 \\
\hline Retail Sales & $0.07^{* *}$ & $0.07^{* *}$ & $0.03^{* *}$ & $0.03^{* *}$ & $-0.07^{\star *}$ & $0.07^{* *}$ & 0.02 & 0.01 & -0.01 \\
\hline Trade Balance & $0.12^{* *}$ & $0.12^{* *}$ & 0.00 & $0.02^{* *}$ & $-0.02^{* *}$ & $0.12^{* *}$ & $0.09^{* *}$ & 0.00 & 0.02 \\
\hline Initial Unemployment & $-0.03^{* *}$ & $-0.03^{* *}$ & -0.01 & $-0.01^{* *}$ & 0.00 & $-0.02^{* *}$ & $-0.02^{* *}$ & 0.01 & 0.01 \\
\hline Wholesales & 0.00 & 0.00 & 0.00 & 0.01 & -0.01 & 0.00 & 0.00 & 0.00 & 0.00 \\
\hline Budget Deficit & $0.05^{* *}$ & 0.03 & & & & 0.01 & & & \\
\hline Current Account & $-0.03^{* *}$ & $-0.02^{*}$ & & & -0.01 & $-0.08^{* *}$ & 0.09 & $0.22^{* *}$ & 0.03 \\
\hline Current Account(US) & -0.01 & & & & & & & & \\
\hline Consumer Confidence & & & & & -0.01 & & & & 0.00 \\
\hline Consumer Price Index & $-0.04^{* *}$ & $0.01^{*}$ & & 0.01 & 0.01 & 0.00 & $-0.10^{* *}$ & -0.01 & 0.01 \\
\hline Exports & $-0.03^{* *}$ & & 0.00 & 0.01 & & 0.02 & & 0.02 & 0.09 \\
\hline Fixed Invest & & & & & 0.00 & & & & \\
\hline Gross Domestic Product & -0.02 & 0.01 & -0.02 & -0.04 & $-0.02^{*}$ & $-0.03^{* *}$ & -0.02 & -0.04 & -0.03 \\
\hline Imports & $-0.03^{* *}$ & & 0.00 & 0.00 & & -0.01 & & & $-0.19^{* *}$ \\
\hline Interest rate & & & & & & & $-0.30^{* *}$ & -0.01 & \\
\hline Industrial production & $-0.01^{*}$ & $-0.03^{* *}$ & & 0.00 & 0.01 & & & & -0.03 \\
\hline Money Supply & 0.00 & & & & & $-0.01^{*}$ & $0.15^{\star *}$ & & \\
\hline Producer Price & $-0.02^{* *}$ & -0.02 & & & 0.01 & 0.00 & 0.01 & & $0.14^{* *}$ \\
\hline Retail Sales & $-0.03^{* *}$ & & & & -0.01 & -0.01 & 0.06 & & \\
\hline Trade Balance & $-0.07^{* *}$ & 0.00 & 0.52 & & -0.01 & & $-0.16^{* *}$ & & 0.00 \\
\hline Initial Unemployment & 0.07 & & & -0.01 & $-0.02^{\star *}$ & $0.02^{*}$ & & & -0.01 \\
\hline Wholesales & & & & & -0.03 & $-0.03^{* *}$ & & & \\
\hline
\end{tabular}




\begin{tabular}{|c|c|c|c|c|c|c|c|c|c|}
\hline Announcements & \multicolumn{3}{|l|}{ Czech } & Korea & Mexico & Poland & $\begin{array}{l}\text { South } \\
\text { Africa }\end{array}$ & Thailand & Turkey \\
\hline \multicolumn{10}{|c|}{ Impact of Contemporaneous News Surprises on Volatility } \\
\hline Business Inventories & 0.00 & $0.02^{* *}$ & 0.00 & 0.00 & 0.01 & 0.00 & $-0.01^{* *}$ & 0.00 & $0.05^{\star *}$ \\
\hline Budget Deficit & -0.01 & 0.01 & 0.01 & $0.11^{* \star}$ & 0.00 & 0.00 & 0.01 & -0.01 & 0.13 \\
\hline Current Account & $-0.02^{* *}$ & 0.00 & $0.02^{* *}$ & 0.01 & 0.00 & $-0.01^{* *}$ & 0.01 & 0.01 & 0.00 \\
\hline Capital Utilization & $0.03^{* *}$ & $0.02^{* *}$ & -0.01 & 0.00 & 0.00 & 0.00 & $-0.02^{* *}$ & $-0.01^{*}$ & -0.02 \\
\hline Consumer Confidence & $0.03^{* *}$ & $0.04^{* *}$ & $0.03^{* *}$ & $0.03^{* *}$ & $0.02^{* *}$ & $0.03^{* *}$ & 0.00 & $0.01^{* *}$ & 0.00 \\
\hline Consumer Credit & 0.00 & 0.00 & $0.01^{*}$ & 0.00 & 0.00 & 0.01 & 0.00 & 0.00 & -0.08 \\
\hline Construction Spending & $0.01^{* *}$ & $0.01^{* \star}$ & $-0.02^{* *}$ & $0.02^{*}$ & 0.01 & 0.00 & 0.01 & 0.00 & 0.00 \\
\hline Consumer Price Index & $0.05^{* *}$ & $0.04^{* *}$ & $0.00^{*}$ & 0.00 & $0.03^{* *}$ & $0.04^{* *}$ & $0.05^{* *}$ & 0.00 & 0.01 \\
\hline Durable Goods Orders & $0.02^{* *}$ & $0.05^{\star *}$ & $0.01^{*}$ & $-0.01^{* *}$ & 0.00 & $0.01^{* *}$ & $0.01^{* *}$ & $0.02^{* *}$ & 0.01 \\
\hline Factory Orders & $0.04^{* *}$ & $0.02^{* *}$ & 0.01 & $0.00^{*}$ & 0.00 & 0.00 & $0.00^{* *}$ & 0.01 & -0.01 \\
\hline Gross Domestic Product & $0.05^{\star *}$ & $0.04^{* *}$ & $0.04^{* *}$ & $0.04^{* *}$ & 0.00 & $0.04^{* *}$ & $0.04^{* *}$ & 0.01 & $0.03^{* *}$ \\
\hline Housing Starts & $0.01^{* *}$ & $0.02^{* *}$ & $0.02^{* *}$ & 0.01 & 0.00 & 0.00 & 0.00 & 0.00 & 0.00 \\
\hline Imports & $0.02^{* \star}$ & $0.01^{* *}$ & 0.00 & $0.02^{*}$ & $0.01^{*}$ & 0.01 & 0.00 & 0.00 & $0.02^{*}$ \\
\hline Interest rate & 0.00 & $0.01^{* *}$ & 0.00 & 0.00 & 0.00 & 0.00 & $0.03^{\star \star}$ & 0.00 & 0.00 \\
\hline Industrial production & 0.00 & $0.00^{* *}$ & 0.00 & 0.00 & 0.00 & $0.02^{* *}$ & 0.00 & -0.01 & 0.00 \\
\hline NAPM & $0.05^{*}$ & 0.05 & $0.42^{* *}$ & 0.10 & 0.05 & $0.82^{* *}$ & 0.06 & 0.24 & 0.02 \\
\hline Leading Indicators & 0.01 & 0.00 & 0.00 & 0.00 & 0.00 & 0.00 & 0.01 & 0.00 & 0.00 \\
\hline New Home Sales & $0.03^{* *}$ & $0.03^{* *}$ & $0.02^{* *}$ & $0.02^{* *}$ & 0.00 & $0.03^{* *}$ & 0.00 & 0.00 & -0.01 \\
\hline Nonfarm Payroll & $0.17^{* *}$ & $0.17^{* *}$ & $-0.01^{* *}$ & $0.09^{* *}$ & $0.05^{* *}$ & $0.16^{* *}$ & $0.18^{* *}$ & $0.01^{* *}$ & $0.10^{* *}$ \\
\hline Personal Spending & $0.02^{* \star}$ & 0.01 & 0.01 & 0.01 & 0.00 & -0.01 & $0.00^{* *}$ & -0.01 & $0.03^{* *}$ \\
\hline Personal Income & $0.01^{* *}$ & 0.00 & 0.00 & 0.00 & $0.01^{* *}$ & $0.05^{* *}$ & 0.01 & 0.00 & 0.01 \\
\hline Producer Price & $0.03^{\star *}$ & $0.03^{* *}$ & $0.02^{* \star}$ & $-0.01^{*}$ & $0.01^{* *}$ & 0.01 & $0.01^{* *}$ & 0.00 & $0.07^{\star *}$ \\
\hline Retail Sales & $0.03^{* *}$ & $0.03^{* *}$ & $0.03^{* *}$ & $0.05^{\star *}$ & $0.03^{* *}$ & $0.04^{* *}$ & $0.00^{*}$ & 0.00 & 0.00 \\
\hline Trade Balance & $0.04^{* *}$ & $0.04^{* *}$ & $0.04^{* *}$ & $0.04^{* *}$ & $0.00^{* *}$ & $0.06^{* *}$ & $0.03^{* *}$ & 0.00 & 0.01 \\
\hline Initial Unemployment & $0.01^{* *}$ & 0.00 & $0.01^{* *}$ & $0.01^{* *}$ & $0.01^{* *}$ & $0.02^{* *}$ & $0.02^{* *}$ & 0.00 & $0.01^{* *}$ \\
\hline Wholesales & $0.01^{* *}$ & 0.01 & 0.00 & $0.02^{* *}$ & 0.00 & $-0.01^{* *}$ & 0.00 & 0.00 & 0.00 \\
\hline Budget Deficit & 0.00 & -0.01 & & & & 0.00 & & & \\
\hline Current Account & 0.00 & 0.01 & & & $-0.02^{*}$ & $0.02^{* *}$ & 0.06 & $0.24^{* *}$ & 0.00 \\
\hline Current Account(US) & $0.03^{* *}$ & & & & & & & & \\
\hline Consumer Confidence & & & & & 0.00 & & & & 0.00 \\
\hline Consumer Price Index & $0.04^{* *}$ & $0.04^{* *}$ & & 0.00 & $0.01^{*}$ & 0.00 & 0.01 & 0.00 & 0.00 \\
\hline Exports & 0.02 & & 0.00 & 0.00 & & 0.00 & & 0.00 & 0.00 \\
\hline Fixed Invest & & & & & 0.00 & & & & \\
\hline Gross Domestic Product & 0.01 & -0.01 & -0.02 & -0.02 & 0.00 & $0.02^{* *}$ & $0.03^{*}$ & -0.01 & 0.00 \\
\hline Imports & $0.02^{* *}$ & & 0.00 & 0.01 & & $0.02^{* *}$ & & & $0.09^{*}$ \\
\hline Interest rate & & & & & & & $0.26^{\star \star}$ & 0.00 & \\
\hline Industrial production & $0.01^{* *}$ & $0.01^{* *}$ & & 0.00 & 0.00 & & & & 0.00 \\
\hline Money Supply & $0.00^{* *}$ & & & & & $0.01^{*}$ & $0.03^{* *}$ & & \\
\hline Producer Price & 0.00 & -0.01 & & & $0.00^{*}$ & 0.01 & $0.02^{*}$ & & $0.19^{* *}$ \\
\hline Retail Sales & 0.00 & & & & $-0.01^{* *}$ & -0.01 & $-0.04^{*}$ & & \\
\hline Trade Balance & $0.03^{* *}$ & 0.01 & 0.17 & & -0.01 & & $0.07^{* *}$ & & -0.02 \\
\hline Initial Unemployment & $-0.04^{* *}$ & & & $0.00^{* *}$ & $0.02^{* *}$ & 0.00 & & & 0.00 \\
\hline Wholesales & & & & & 0.00 & $0.03^{* *}$ & & & \\
\hline
\end{tabular}




\begin{tabular}{|c|c|c|c|c|c|c|c|c|c|}
\hline Announcements & \multicolumn{4}{|l|}{ Czech } & \multirow[t]{2}{*}{ Mexico } & Poland & $\begin{array}{l}\text { South } \\
\text { Africa }\end{array}$ & Thailand & Turkey \\
\hline \multicolumn{9}{|c|}{ Impact of Cumulated news Surprises on volatility } & \\
\hline Business Inventories & 0.01 & $0.04^{* *}$ & -0.01 & -0.01 & 0.01 & 0.00 & $0.04^{* *}$ & 0.00 & $0.10^{* *}$ \\
\hline Budget Deficit & -0.02 & 0.06 & -0.04 & $0.11^{* *}$ & -0.01 & -0.01 & 0.05 & -0.01 & -0.12 \\
\hline Current Account & $0.03^{* *}$ & 0.00 & $-0.05^{\star \star}$ & 0.03 & 0.00 & $0.05^{\star *}$ & 0.00 & 0.01 & -0.02 \\
\hline Capital Utilization & $0.11^{* *}$ & $0.05^{* *}$ & 0.02 & 0.01 & 0.01 & 0.01 & $0.08^{* *}$ & $-0.07^{*}$ & -0.02 \\
\hline Consumer Confidence & $0.03^{* *}$ & $0.10^{\star *}$ & $0.13^{* *}$ & $0.07^{* *}$ & $0.05^{\star *}$ & $0.12^{* *}$ & 0.01 & $0.02^{* *}$ & -0.04 \\
\hline Consumer Credit & 0.00 & 0.00 & $-0.04^{*}$ & 0.00 & -0.01 & -0.04 & 0.01 & 0.00 & 0.75 \\
\hline Construction Spending & $0.00^{* *}$ & $0.00^{\star *}$ & $0.09^{* *}$ & $0.01^{*}$ & 0.02 & 0.00 & 0.03 & 0.00 & 0.00 \\
\hline Consumer Price Index & $0.13^{* *}$ & $0.08^{* *}$ & $0.06^{*}$ & 0.01 & $0.09^{* *}$ & $0.05^{\star *}$ & $0.12^{* *}$ & 0.00 & 0.08 \\
\hline Durable Goods Orders & $0.17^{\star *}$ & $0.20^{* \star}$ & $0.05^{*}$ & $0.05^{\star *}$ & 0.00 & $0.07^{* *}$ & $0.13^{* *}$ & $0.02^{* *}$ & 0.03 \\
\hline Factory Orders & $0.13^{* *}$ & $0.06^{* *}$ & 0.02 & $0.02^{*}$ & 0.01 & 0.02 & $0.07^{* *}$ & -0.02 & -0.05 \\
\hline Gross Domestic Product & $0.06^{* *}$ & $0.02^{* *}$ & $0.05^{\star *}$ & $0.04^{* *}$ & 0.00 & $0.11^{* *}$ & $0.08^{* *}$ & 0.03 & $-0.02^{* *}$ \\
\hline Housing Starts & $0.02^{* *}$ & $0.03^{* *}$ & $0.04^{* *}$ & 0.00 & 0.00 & 0.00 & 0.01 & 0.00 & 0.01 \\
\hline Imports & $0.14^{* *}$ & $0.06^{* *}$ & -0.01 & $0.04^{*}$ & $0.02^{*}$ & 0.00 & -0.01 & -0.01 & $0.01^{*}$ \\
\hline Interest rate & -0.01 & $0.01^{* *}$ & 0.00 & 0.00 & 0.01 & 0.01 & $-0.09^{* *}$ & -0.01 & 0.00 \\
\hline Industrial production & 0.00 & $0.04^{* *}$ & 0.01 & -0.01 & 0.00 & $0.06^{* *}$ & 0.01 & -0.03 & -0.03 \\
\hline NAPM & $-0.11^{*}$ & 0.08 & $2.57^{* *}$ & 0.10 & -0.11 & $2.43^{* *}$ & 0.46 & 0.03 & 0.00 \\
\hline Leading Indicators & 0.01 & 0.00 & 0.00 & 0.00 & 0.00 & 0.02 & 0.01 & 0.01 & 0.00 \\
\hline New Home Sales & $0.10^{* \star}$ & $0.06^{\star \star}$ & $0.05^{\star *}$ & $0.03^{* *}$ & -0.01 & $0.08^{* *}$ & 0.01 & 0.00 & -0.01 \\
\hline Nonfarm Payroll & $0.26^{* *}$ & $0.32^{* *}$ & $0.16^{* *}$ & $0.39^{* *}$ & $0.19^{* *}$ & $0.37^{* *}$ & $0.38^{* *}$ & $0.05^{* *}$ & $0.16^{* *}$ \\
\hline Personal Spending & $0.08^{* *}$ & 0.00 & 0.01 & 0.02 & 0.00 & -0.01 & $0.09^{* *}$ & -0.02 & $0.03^{* *}$ \\
\hline Personal Income & $0.03^{* *}$ & 0.00 & 0.00 & 0.00 & $0.03^{* *}$ & $0.14^{* *}$ & -0.01 & 0.00 & 0.02 \\
\hline Producer Price & $0.03^{* \star}$ & $0.06^{* \star}$ & $0.04^{* \star}$ & $0.04^{*}$ & $0.01^{* *}$ & 0.01 & $0.10^{* *}$ & -0.01 & $0.08^{* *}$ \\
\hline Retail Sales & $0.09^{* *}$ & $0.05^{\star *}$ & $0.06^{* *}$ & $0.13^{* *}$ & $0.03^{* *}$ & $0.16^{* *}$ & $0.06^{*}$ & 0.00 & 0.01 \\
\hline Trade Balance & $0.05^{\star *}$ & $0.06^{* *}$ & $0.01^{* *}$ & $0.25^{\star *}$ & $0.02^{* *}$ & $0.11^{* *}$ & $0.11^{* *}$ & -0.03 & 0.02 \\
\hline Initial Unemployment & $0.03^{* *}$ & 0.00 & $-0.01^{* *}$ & $0.01^{* *}$ & $0.04^{* *}$ & $0.12^{* *}$ & $0.07^{* *}$ & 0.00 & $0.01^{* *}$ \\
\hline Wholesales & $0.02^{* *}$ & 0.00 & 0.00 & $0.00^{* *}$ & -0.02 & $0.04^{* *}$ & 0.01 & -0.01 & -0.02 \\
\hline Budget Deficit & 0.00 & -0.01 & & & & 0.03 & & & \\
\hline Current Account & 0.00 & -0.01 & & & $-0.06^{*}$ & $0.06^{* *}$ & 0.09 & $0.40^{* *}$ & 0.01 \\
\hline Current Account(US) & $0.11^{* *}$ & & & & & & & & \\
\hline Consumer Confidence & & & & & -0.01 & & & & 0.00 \\
\hline Consumer Price Index & $0.14^{* *}$ & $0.12^{* *}$ & & 0.01 & $-0.01^{*}$ & 0.00 & 0.00 & 0.00 & 0.00 \\
\hline Exports & 0.03 & & 0.02 & 0.00 & & -0.03 & & -0.01 & 0.01 \\
\hline Fixed Invest & & & & & 0.00 & & & & \\
\hline Gross Domestic Product & -0.01 & 0.01 & -0.03 & -0.04 & 0.00 & $0.09^{* *}$ & $0.07^{*}$ & 0.01 & -0.16 \\
\hline Imports & $0.12^{* *}$ & & 0.04 & 0.01 & & $0.13^{* *}$ & & & $0.23^{*}$ \\
\hline Interest rate & & & & & & & $0.31^{* *}$ & -0.03 & \\
\hline Industrial production & $0.05^{\star *}$ & $0.07^{* *}$ & & 0.00 & 0.01 & & & & 0.00 \\
\hline Money Supply & $0.00^{* *}$ & & & & & $0.04^{*}$ & $0.07^{* *}$ & & \\
\hline Producer Price & 0.00 & -0.01 & & & $0.07^{*}$ & 0.01 & $0.02^{*}$ & & $0.49^{* *}$ \\
\hline Retail Sales & 0.00 & & & & $-0.07^{* *}$ & 0.01 & $0.04^{*}$ & & \\
\hline Trade Balance & $0.10^{* *}$ & 0.01 & 1.96 & & -0.02 & & $0.02^{* *}$ & & -0.02 \\
\hline Initial Unemployment & $-0.07^{\star *}$ & & & $0.19^{* *}$ & $0.01^{* *}$ & -0.01 & & & 0.01 \\
\hline Wholesales & & & & & 0.04 & $0.18^{* *}$ & & & \\
\hline
\end{tabular}




\section{Appendix 5. Return and Volatility Response with Announcement Dummy}

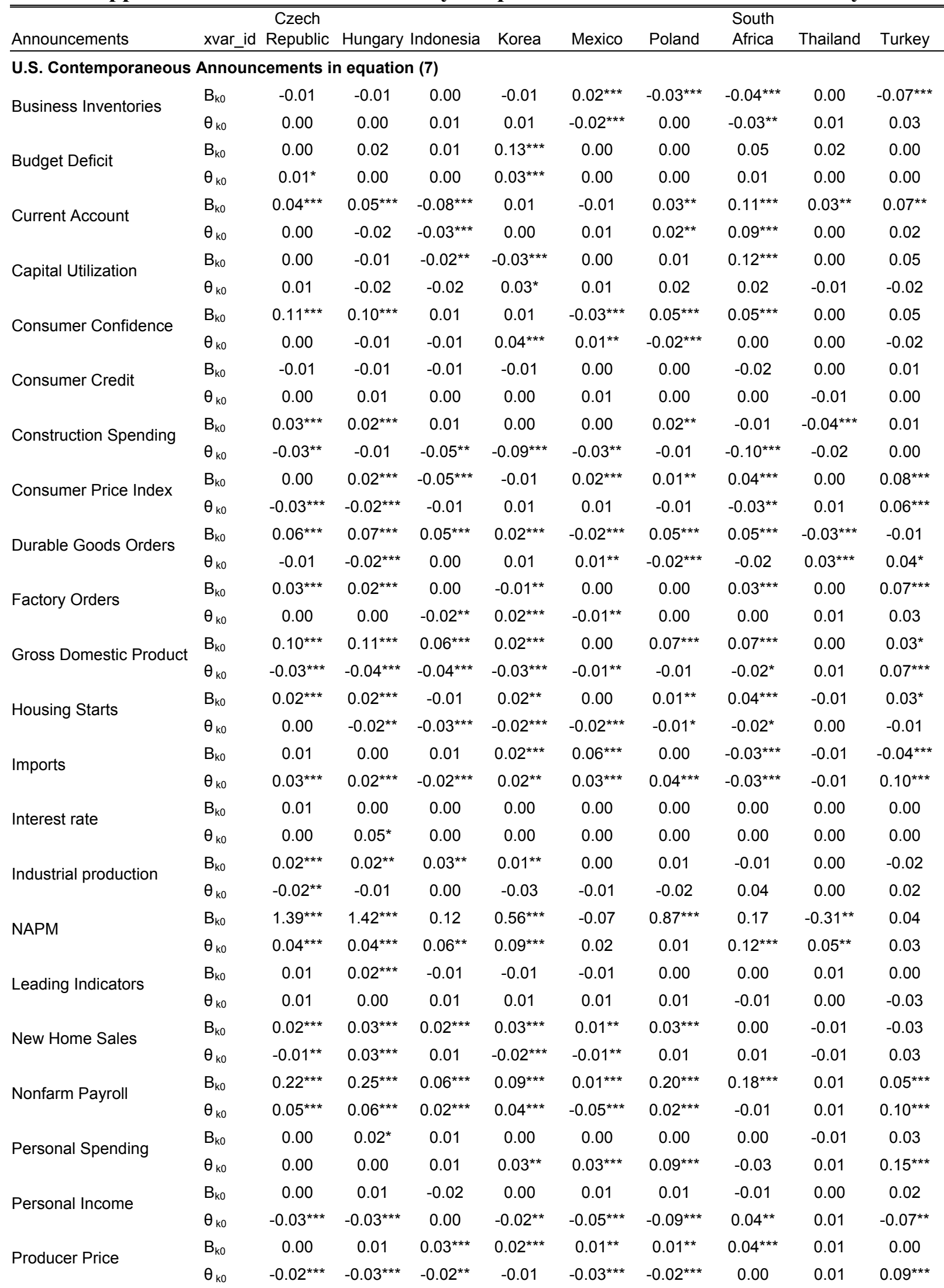




\begin{tabular}{|c|c|c|c|c|c|c|c|c|c|c|}
\hline Announcements & xvar_id & $\begin{array}{c}\text { Czech } \\
\text { Republic }\end{array}$ & Hungary & Indonesia & Korea & Mexico & Poland & $\begin{array}{l}\text { South } \\
\text { Africa }\end{array}$ & Thailand & Turkey \\
\hline \multicolumn{11}{|c|}{ U.S. Contemporaneous Announcements in equation (7) } \\
\hline \multirow{2}{*}{ Retail Sales } & $\mathrm{B}_{\mathrm{k} 0}$ & $0.09^{* * *}$ & $0.08^{* * *}$ & $0.03^{* * *}$ & $0.03^{* * *}$ & $-0.07^{* * *}$ & $0.08^{* * *}$ & $0.03^{* *}$ & 0.01 & -0.01 \\
\hline & $\theta_{\mathrm{k} 0}$ & 0.00 & 0.00 & 0.00 & 0.00 & $0.02^{\star \star *}$ & 0.00 & $-0.06^{\star \star *}$ & 0.01 & $-0.04^{* *}$ \\
\hline \multirow{2}{*}{ Trade Balance } & $\mathrm{B}_{\mathrm{k} 0}$ & $0.12^{* * *}$ & $0.11^{* * *}$ & 0.00 & $0.02^{* \star *}$ & $-0.02^{* * *}$ & $0.11^{* * *}$ & $0.10^{* * *}$ & 0.00 & $0.03^{*}$ \\
\hline & $\theta_{\mathrm{k} 0}$ & 0.01 & 0.00 & $-0.02^{* *}$ & $0.02^{* * *}$ & 0.00 & $0.01^{* *}$ & 0.01 & 0.01 & $0.05^{\star *}$ \\
\hline \multirow{2}{*}{ Initial Unemployment } & $\mathrm{B}_{\mathrm{k} 0}$ & $-0.04^{* * *}$ & $-0.03^{* * *}$ & $-0.01^{\text {** }}$ & $-0.02^{* * *}$ & 0.00 & $-0.02^{\star \star *}$ & $-0.02^{\star \star \star}$ & 0.01 & 0.01 \\
\hline & $\theta_{\mathrm{k} 0}$ & $-0.01^{\star \star \star}$ & $-0.01^{\star *}$ & 0.00 & 0.00 & 0.00 & $-0.01^{\star \star \star}$ & -0.01 & $-0.01^{*}$ & 0.01 \\
\hline \multirow{2}{*}{ Wholesales } & $\mathrm{B}_{\mathrm{k} 0}$ & 0.00 & 0.00 & 0.00 & 0.01 & -0.01 & 0.00 & 0.01 & 0.00 & 0.01 \\
\hline & $\theta_{\mathrm{k} 0}$ & $-0.01^{* * *}$ & $-0.01^{*}$ & -0.01 & $-0.04^{* * *}$ & 0.00 & $-0.02^{* *}$ & -0.01 & 0.00 & -0.02 \\
\hline \multicolumn{11}{|c|}{ Domestic Contemporaneous Announcements in equation (7) } \\
\hline \multirow{2}{*}{ Budget Deficit } & $\mathrm{B}_{\mathrm{k} 0}$ & 0.01 & 0.02 & & & & 0.00 & & & \\
\hline & $\theta_{\mathrm{k} 0}$ & $0.11^{* * *}$ & 0.00 & & & & $-0.02^{* *}$ & & & \\
\hline \multirow{2}{*}{ Current Account } & $\mathrm{B}_{\mathrm{k} 0}$ & $-0.02^{\star *}$ & $-0.01^{* *}$ & & & 0.00 & $-0.08^{* \star *}$ & 0.00 & $0.18^{* * *}$ & 0.02 \\
\hline & $\theta_{\mathrm{k} 0}$ & 0.01 & $0.02^{* *}$ & & & 0.00 & -0.01 & $-0.38^{* *}$ & $-0.07^{\star \star \star}$ & -0.02 \\
\hline \multirow{2}{*}{ Current Account(US) } & $\mathrm{B}_{\mathrm{k} 0}$ & -0.01 & & & & & & & & \\
\hline & $\theta_{\mathrm{k} 0}$ & $0.04^{* * *}$ & & & & & & & & \\
\hline \multirow{2}{*}{ Consumer Confidence } & $\mathrm{B}_{\mathrm{k} 0}$ & & & & & 0.00 & & & & 0.00 \\
\hline & $\theta_{\mathrm{k} 0}$ & & & & & 0.01 & & & & 0.00 \\
\hline \multirow{2}{*}{ Consumer Price Index } & $\mathrm{B}_{\mathrm{k} 0}$ & -0.01 & $0.02^{* * *}$ & & 0.01 & 0.01 & -0.01 & $-0.09^{* * *}$ & -0.01 & 0.03 \\
\hline & $\theta_{\mathrm{k} 0}$ & -0.01 & $-0.04^{* * *}$ & & -0.01 & 0.00 & $-0.02^{*}$ & -0.01 & 0.00 & $-0.13^{*}$ \\
\hline \multirow{2}{*}{ Exports } & $\mathrm{B}_{\mathrm{k} 0}$ & -0.01 & & $-0.05^{\star * *}$ & 0.00 & & $0.02^{* *}$ & & 0.09 & -0.01 \\
\hline & $\theta_{\mathrm{k} 0}$ & -0.15 & & 0.00 & 0.00 & & 0.00 & & 0.01 & $-0.18^{\star * *}$ \\
\hline \multirow{2}{*}{ Fixed Invest } & $\mathrm{B}_{\mathrm{k} 0}$ & & & & & 0.00 & & & & \\
\hline & $\theta_{\mathrm{k} 0}$ & & & & & 0.00 & & & & \\
\hline \multirow{2}{*}{ Gross Domestic Product } & $\mathrm{B}_{\mathrm{k} 0}$ & -0.01 & 0.01 & -0.03 & -0.05 & $-0.02^{\star *}$ & $-0.03^{* *}$ & -0.03 & $-0.08^{* * *}$ & -0.03 \\
\hline & $\theta_{\mathrm{k} 0}$ & $-0.03^{* * *}$ & 0.00 & 0.01 & -0.01 & -0.01 & 0.00 & $-0.08^{* *}$ & $-0.06^{* \star *}$ & 0.01 \\
\hline \multirow{2}{*}{ Imports } & $\mathrm{B}_{\mathrm{k} 0}$ & -0.07 & & $0.04^{* *}$ & 0.00 & & -0.01 & & & $-0.10^{*}$ \\
\hline & $\theta_{\mathrm{k} 0}$ & 0.12 & & $0.22^{* \star *}$ & 0.00 & & 0.00 & & & 0.00 \\
\hline \multirow{2}{*}{ Interest rate } & $\mathrm{B}_{\mathrm{k} 0}$ & & & & & & & $-0.14^{\star \star *}$ & -0.01 & \\
\hline & $\theta_{\mathrm{k} 0}$ & & & & & & & $0.58^{* * *}$ & 0.00 & \\
\hline \multirow{2}{*}{ Industrial production } & $\mathrm{B}_{\mathrm{k} 0}$ & -0.01 & $-0.03^{* * *}$ & & 0.00 & 0.01 & & & & -0.03 \\
\hline & $\theta_{\mathrm{k} 0}$ & -0.01 & 0.00 & & 0.00 & 0.01 & & & & -0.02 \\
\hline \multirow{2}{*}{ Money Supply } & $\mathrm{B}_{\mathrm{k} 0}$ & 0.02 & & & & & -0.01 & $0.09^{* * *}$ & & \\
\hline & $\theta_{\mathrm{k} 0}$ & -0.15 & & & & & 0.00 & $0.10^{* * *}$ & & \\
\hline \multirow{2}{*}{ Producer Price } & $\mathrm{B}_{\mathrm{k} 0}$ & $-0.02^{\star \star *}$ & 0.01 & & & 0.01 & 0.00 & 0.01 & & $0.17^{\star \star \star}$ \\
\hline & $\theta_{\mathrm{k} 0}$ & $-0.01^{\text {** }}$ & $-0.02^{\star *}$ & & & -0.01 & $-0.09^{\star \star *}$ & $0.03^{*}$ & & $0.24^{\star \star \star}$ \\
\hline \multirow{2}{*}{ Retail Sales } & $\mathrm{B}_{\mathrm{k} 0}$ & $-0.03^{\star \star *}$ & & & & -0.01 & -0.01 & 0.12 & & \\
\hline & $\theta_{\mathrm{k} 0}$ & -0.01 & & & & 0.01 & $-0.03^{* * *}$ & 0.00 & & \\
\hline \multirow{2}{*}{ Trade Balance } & $\mathrm{B}_{\mathrm{k} 0}$ & $-0.07^{\star \star *}$ & -0.01 & $4.97^{\star \star \star}$ & & $-0.01^{\star *}$ & & $-0.17^{\star \star *}$ & & 0.03 \\
\hline & $\theta_{\mathrm{k} 0}$ & $-0.03^{* * *}$ & 0.01 & $-0.21^{* * *}$ & & 0.00 & & $0.04^{* \star *}$ & & 0.04 \\
\hline Initial Unemployment & $\mathrm{B}_{\mathrm{k} 0}$ & 0.01 & & & -0.01 & $-0.01^{* * *}$ & $0.02^{* *}$ & & & -0.01 \\
\hline & $\theta_{\mathrm{k} 0}$ & $0.02^{\star * \star}$ & & & 0.00 & -0.01 & $0.03^{\star \star}$ & & & -0.01 \\
\hline Wholesales & $\mathrm{B}_{\mathrm{k} 0}$ & & & & & $-0.03^{*}$ & $-0.03^{\star \star *}$ & & & \\
\hline & $\theta_{\mathrm{k} 0}$ & & & & & 0.00 & $0.06^{* * *}$ & & & \\
\hline
\end{tabular}




\begin{tabular}{|c|c|c|c|c|c|c|c|c|c|c|}
\hline Announcements & xvar_id & $\begin{array}{c}\text { Czech } \\
\text { Republic }\end{array}$ & Hungary & Indonesia & Korea & Mexico & Poland & $\begin{array}{l}\text { South } \\
\text { Africa }\end{array}$ & Thailand & Turkey \\
\hline \multicolumn{11}{|c|}{ U.S. Contemporaneous Announcements in equation (8) } \\
\hline \multirow{2}{*}{ Business Inventories } & $\mathrm{B}_{\mathrm{k} 0}$ & 0.00 & -0.01 & 0.01 & 0.01 & 0.01 & 0.01 & $-0.01^{* *}$ & 0.00 & $0.06^{* \star *}$ \\
\hline & $\theta_{\mathrm{k} 0}$ & $0.03^{* * *}$ & $0.04^{* * *}$ & 0.02 & 0.02 & -0.01 & 0.02 & $0.02^{*}$ & -0.01 & -0.04 \\
\hline \multirow{2}{*}{ Budget Deficit } & $\mathrm{B}_{\mathrm{k} 0}$ & 0.00 & 0.01 & 0.01 & $0.15^{\star \star \star}$ & 0.00 & 0.00 & 0.01 & 0.03 & 0.11 \\
\hline & $\theta_{\mathrm{k} 0}$ & 0.00 & 0.00 & -0.01 & -0.01 & 0.00 & -0.01 & -0.02 & -0.01 & 0.00 \\
\hline \multirow{2}{*}{ Current Account } & $\mathrm{B}_{\mathrm{k} 0}$ & 0.01 & -0.02 & $0.03^{* *}$ & -0.02 & 0.00 & $-0.02^{* *}$ & 0.01 & 0.02 & -0.02 \\
\hline & $\theta_{\mathrm{k} 0}$ & -0.01 & $0.03^{*}$ & $0.04^{* \star}$ & $0.03^{*}$ & 0.01 & $0.03^{*}$ & 0.00 & 0.00 & 0.01 \\
\hline \multirow{2}{*}{ Capital Utilization } & $\mathrm{B}_{\mathrm{k} 0}$ & -0.01 & -0.02 & 0.01 & 0.01 & -0.01 & 0.00 & -0.02 & 0.00 & -0.01 \\
\hline & $\theta_{\mathrm{k} 0}$ & 0.01 & 0.00 & 0.01 & 0.02 & 0.02 & 0.01 & 0.03 & 0.01 & -0.03 \\
\hline \multirow{2}{*}{ Consumer Confidence } & $\mathrm{B}_{\mathrm{k} 0}$ & $0.02^{* * *}$ & $0.03^{* * *}$ & 0.00 & 0.00 & $0.01^{* *}$ & 0.01 & 0.00 & $0.01^{* * *}$ & 0.00 \\
\hline & $\theta_{\mathrm{k} 0}$ & $0.03^{* * *}$ & $0.02^{* * *}$ & $0.03^{* *}$ & $0.04^{* *}$ & 0.00 & $0.04^{* * *}$ & 0.01 & -0.01 & 0.00 \\
\hline \multirow{2}{*}{ Consumer Credit } & $\mathrm{B}_{\mathrm{k} 0}$ & -0.01 & -0.01 & 0.00 & -0.01 & 0.00 & 0.00 & 0.00 & -0.01 & -0.01 \\
\hline & $\theta_{\mathrm{k} 0}$ & 0.01 & 0.00 & -0.01 & 0.00 & 0.00 & 0.00 & 0.01 & 0.00 & -0.07 \\
\hline \multirow{2}{*}{ Construction Spending } & $\mathrm{B}_{\mathrm{k} 0}$ & $-0.01^{*}$ & 0.00 & 0.00 & 0.01 & 0.00 & 0.00 & -0.01 & 0.00 & 0.00 \\
\hline & $\theta_{\mathrm{k} 0}$ & $0.03^{* * *}$ & $0.03^{* *}$ & 0.00 & 0.01 & $0.02^{*}$ & 0.01 & 0.00 & 0.00 & 0.02 \\
\hline \multirow{2}{*}{ Consumer Price Index } & $\mathrm{B}_{\mathrm{k} 0}$ & 0.01 & 0.00 & $0.00^{* *}$ & $-0.02^{* *}$ & 0.00 & $-0.03^{*}$ & -0.03 & 0.00 & $-0.01^{*}$ \\
\hline & $\theta_{\mathrm{k} 0}$ & $0.04^{* * *}$ & $0.05^{\star * *}$ & $0.03^{\star * *}$ & $0.03^{*}$ & $0.05^{* \star *}$ & $0.09^{* * *}$ & $0.09^{* * *}$ & 0.01 & $0.10^{\star * *}$ \\
\hline \multirow{2}{*}{ Durable Goods Orders } & $\mathrm{B}_{\mathrm{k} 0}$ & -0.01 & 0.00 & 0.00 & -0.01 & 0.00 & 0.00 & 0.00 & $0.03^{* * *}$ & 0.01 \\
\hline & $\theta_{\mathrm{k} 0}$ & $0.05^{\star \star \star}$ & $0.05^{\star \star \star}$ & $0.04^{\star \star *}$ & $0.03^{* \star *}$ & $0.02^{* \star *}$ & $0.04^{* \star *}$ & 0.01 & 0.00 & -0.01 \\
\hline \multirow{2}{*}{ Factory Orders } & $\mathrm{B}_{\mathrm{k} 0}$ & -0.01 & $-0.01^{* *}$ & $-0.03^{* *}$ & 0.00 & 0.00 & 0.00 & 0.00 & 0.00 & 0.01 \\
\hline & $\theta_{\mathrm{k} 0}$ & $0.06^{* * *}$ & $0.05^{\star * *}$ & $0.05^{\star * *}$ & $0.05^{\star \star *}$ & 0.01 & $0.03^{\star * *}$ & $0.02^{*}$ & 0.00 & $-0.03^{*}$ \\
\hline \multirow{2}{*}{ Gross Domestic Product } & $\mathrm{B}_{\mathrm{k} 0}$ & $0.02^{* * *}$ & $0.03^{* * *}$ & $0.03^{* * *}$ & $0.02^{* *}$ & 0.00 & $0.01^{*}$ & $0.03^{* *}$ & $0.02^{\star * *}$ & -0.01 \\
\hline & $\theta_{\mathrm{k} 0}$ & $0.04^{* * *}$ & $0.03^{* * *}$ & 0.01 & 0.01 & $0.02^{* * *}$ & $0.03^{* * *}$ & 0.01 & $-0.02^{*}$ & $0.06^{\star \star *}$ \\
\hline \multirow{2}{*}{ Housing Starts } & $\mathrm{B}_{\mathrm{k} 0}$ & $-0.01^{* * *}$ & -0.01 & 0.00 & $-0.03^{\star * *}$ & 0.00 & 0.00 & -0.01 & 0.00 & 0.00 \\
\hline & $\theta_{\mathrm{k} 0}$ & $0.04^{* * *}$ & $0.03^{* * *}$ & 0.02 & $0.06^{\star * *}$ & 0.01 & $0.02^{* *}$ & 0.01 & 0.00 & 0.02 \\
\hline \multirow{2}{*}{ Imports } & $\mathrm{B}_{\mathrm{k} 0}$ & -0.01 & 0.00 & 0.00 & 0.01 & $-0.01^{*}$ & $0.02^{\star *}$ & 0.00 & 0.00 & 0.01 \\
\hline & $\theta_{\mathrm{k} 0}$ & 0.01 & 0.00 & $0.03^{\star *}$ & 0.00 & $0.02^{* \star}$ & 0.00 & $0.04^{* * *}$ & 0.00 & 0.03 \\
\hline \multirow{2}{*}{ Interest rate } & $\mathrm{B}_{\mathrm{k} 0}$ & 0.00 & 0.00 & 0.00 & 0.00 & 0.00 & 0.00 & 0.00 & 0.00 & 0.00 \\
\hline & $\theta_{\mathrm{k} 0}$ & -0.03 & $0.06^{* *}$ & 0.00 & 0.00 & -0.03 & -0.03 & -0.05 & -0.04 & 0.00 \\
\hline \multirow{2}{*}{ Industrial production } & $\mathrm{B}_{\mathrm{k} 0}$ & 0.00 & 0.00 & 0.00 & 0.00 & 0.00 & 0.00 & 0.00 & 0.00 & -0.01 \\
\hline & $\theta_{\mathrm{k} 0}$ & $0.03^{* * *}$ & $0.03^{* * *}$ & -0.01 & 0.02 & 0.00 & -0.01 & 0.01 & -0.02 & 0.03 \\
\hline \multirow{2}{*}{ NAPM } & $\mathrm{B}_{\mathrm{k} 0}$ & $0.08^{* * *}$ & -0.03 & 0.03 & $-0.46^{\star *}$ & 0.01 & 0.06 & -0.04 & 0.17 & -0.26 \\
\hline & $\theta_{\mathrm{k} 0}$ & $0.03^{\star * *}$ & $0.04^{* * *}$ & 0.04 & $0.06^{\star *}$ & -0.01 & 0.03 & 0.02 & 0.00 & 0.01 \\
\hline \multirow{2}{*}{ Leading Indicators } & $\mathrm{B}_{\mathrm{k} 0}$ & $-0.01^{*}$ & 0.00 & 0.00 & 0.00 & 0.00 & 0.00 & -0.01 & 0.00 & 0.01 \\
\hline & $\theta_{\mathrm{k} 0}$ & $0.04^{\star * *}$ & $0.02^{* * *}$ & 0.01 & $0.04^{\star * *}$ & 0.01 & 0.00 & 0.03 & -0.01 & -0.04 \\
\hline \multirow{2}{*}{ New Home Sales } & $\mathrm{B}_{\mathrm{k} 0}$ & $-0.02^{* * *}$ & $-0.02^{* *}$ & 0.00 & -0.01 & 0.00 & 0.01 & 0.00 & 0.00 & 0.01 \\
\hline & $\theta_{\mathrm{k} 0}$ & $0.07^{* \star *}$ & $0.05^{\star * *}$ & $0.03^{\star *}$ & $0.06^{* * *}$ & 0.01 & $0.02^{* * *}$ & $0.03^{* * *}$ & 0.00 & -0.03 \\
\hline \multirow{2}{*}{ Nonfarm Payroll } & $\mathrm{B}_{\mathrm{k} 0}$ & $0.07^{* * *}$ & $0.06^{* * *}$ & 0.00 & $0.02^{*}$ & $0.02^{* * *}$ & $0.08^{* * *}$ & $0.12^{\star * *}$ & 0.01 & $0.04^{* *}$ \\
\hline & $\theta_{\mathrm{k} 0}$ & $0.17^{* * *}$ & $0.16^{* * *}$ & $0.08^{* * *}$ & $0.08^{* * *}$ & $0.06^{* * *}$ & $0.12^{* * *}$ & $0.08^{* * *}$ & 0.00 & $0.07^{* * *}$ \\
\hline \multirow{2}{*}{ Personal Spending } & $\mathrm{B}_{\mathrm{k} 0}$ & 0.00 & 0.00 & 0.00 & 0.01 & -0.01 & -0.01 & 0.00 & -0.01 & 0.00 \\
\hline & $\theta_{\mathrm{k} 0}$ & -0.01 & 0.01 & -0.01 & -0.01 & -0.01 & -0.01 & 0.00 & 0.00 & 0.00 \\
\hline Personal Income & $\mathrm{B}_{\mathrm{k} 0}$ & 0.00 & 0.00 & -0.01 & 0.00 & -0.01 & 0.01 & 0.00 & 0.01 & -0.01 \\
\hline & $\theta_{\mathrm{k} 0}$ & $0.04^{* * *}$ & $0.02^{* *}$ & 0.02 & $0.03^{* *}$ & $0.03^{* * *}$ & $0.05^{\star * *}$ & $0.04^{* *}$ & -0.01 & $0.05^{*}$ \\
\hline & $\mathrm{B}_{\mathrm{k} 0}$ & $-0.02^{\star \star *}$ & $-0.02^{* \star *}$ & 0.00 & 0.00 & $-0.01^{*}$ & 0.00 & 0.00 & 0.00 & -0.01 \\
\hline rioducer rilce & $\theta_{\mathrm{k} 0}$ & $0.06^{\star * *}$ & $0.06^{* * *}$ & $0.04^{\star * \star}$ & 0.02 & $0.03^{* * *}$ & $0.03^{\star *}$ & $0.04^{\star * *}$ & -0.01 & $0.08^{\star * *}$ \\
\hline
\end{tabular}




\begin{tabular}{|c|c|c|c|c|c|c|c|c|c|c|}
\hline Announcements & xvar_id & $\begin{array}{c}\text { Czech } \\
\text { Republic }\end{array}$ & Hungary & Indonesia & Korea & Mexico & Poland & $\begin{array}{l}\text { South } \\
\text { Africa }\end{array}$ & Thailand & Turkey \\
\hline \multirow{2}{*}{ Retail Sales } & $\mathrm{B}_{\mathrm{k} 0}$ & $0.01^{*}$ & 0.01 & 0.01 & 0.02 & $0.03^{* * *}$ & $0.02^{* * *}$ & 0.00 & 0.00 & $-0.04^{*}$ \\
\hline & $\theta_{\mathrm{k} 0}$ & $0.04^{\star * *}$ & $0.04^{* * *}$ & 0.01 & $0.02^{* *}$ & $0.03^{\star * *}$ & $0.02^{* *}$ & $0.06^{\star * *}$ & 0.00 & 0.04 \\
\hline \multirow{2}{*}{ Trade Balance } & $\mathrm{B}_{\mathrm{k} 0}$ & $0.02^{* \star *}$ & $0.02^{\star \star \star}$ & 0.01 & $0.03^{\star \star \star}$ & 0.00 & $0.03^{\star \star \star}$ & $0.03^{\star \star \star}$ & $-0.01^{* *}$ & -0.03 \\
\hline & $\theta_{\mathrm{k} 0}$ & $0.04^{* * *}$ & $0.04^{* * *}$ & $0.05^{\star * *}$ & 0.00 & $0.02^{* *}$ & $0.04^{* * *}$ & 0.02 & $0.03^{* * *}$ & $0.04^{*}$ \\
\hline \multirow{2}{*}{ Initial Unemployment } & $\mathrm{B}_{\mathrm{k} 0}$ & 0.00 & 0.00 & 0.01 & 0.00 & 0.00 & -0.01 & 0.00 & 0.00 & 0.00 \\
\hline & $\theta_{\mathrm{k} 0}$ & $0.02^{* * *}$ & $0.02^{* * *}$ & 0.01 & $0.02^{* * *}$ & $0.01^{* * *}$ & $0.03^{* * *}$ & $0.01^{* *}$ & 0.00 & 0.01 \\
\hline \multirow{2}{*}{ Wholesales } & $\mathrm{B}_{\mathrm{k} 0}$ & -0.01 & $-0.01^{*}$ & 0.00 & 0.00 & 0.00 & 0.01 & 0.00 & -0.01 & 0.00 \\
\hline & $\theta_{\mathrm{k} 0}$ & $0.04^{* * *}$ & $0.04^{* \star *}$ & 0.02 & 0.02 & $0.02^{\star \star \star}$ & $0.02^{*}$ & $0.03^{\star \star *}$ & 0.02 & -0.03 \\
\hline \multicolumn{11}{|c|}{ Domestic Contemporaneous Announcements in equation (8) } \\
\hline \multirow{2}{*}{ Budget Deficit } & $\mathrm{B}_{\mathrm{k} 0}$ & 0.01 & $-0.02^{*}$ & & & & 0.00 & & & \\
\hline & $\theta_{\mathrm{k} 0}$ & -0.01 & $0.03^{* *}$ & & & & $0.04^{* * *}$ & & & \\
\hline \multirow{2}{*}{ Current Account } & $\mathrm{B}_{\mathrm{k} 0}$ & $-0.01^{*}$ & $0.02^{* * *}$ & & & -0.01 & -0.01 & -0.11 & $0.37^{* * *}$ & 0.00 \\
\hline & $\theta_{\mathrm{k} 0}$ & 0.01 & $0.03^{* * *}$ & & & 0.00 & -0.04 & 0.02 & $-0.05^{\star *}$ & 0.01 \\
\hline \multirow{2}{*}{ Current Account(US) } & $\mathrm{B}_{\mathrm{k} 0}$ & 0.01 & & & & & & & & \\
\hline & $\theta_{\mathrm{k} 0}$ & 0.01 & & & & & & & & \\
\hline \multirow{2}{*}{ Consumer Confidence } & $\mathrm{B}_{\mathrm{k} 0}$ & & & & & 0.00 & & & & 0.00 \\
\hline & $\theta_{\mathrm{k} 0}$ & & & & & -0.01 & & & & 0.00 \\
\hline \multirow{2}{*}{ Consumer Price Index } & $\mathrm{B}_{\mathrm{k} 0}$ & $-0.02^{*}$ & 0.01 & & 0.00 & 0.00 & -0.01 & 0.01 & 0.00 & -0.01 \\
\hline & $\theta_{\mathrm{k} 0}$ & $0.04^{\star \star *}$ & $0.03^{* \star *}$ & & -0.01 & 0.00 & $0.02^{*}$ & 0.02 & 0.00 & -0.01 \\
\hline \multirow{2}{*}{ Exports } & $\mathrm{B}_{\mathrm{k} 0}$ & 0.01 & & 0.00 & 0.00 & & 0.00 & & 0.00 & -0.07 \\
\hline & $\theta_{\mathrm{k} 0}$ & -0.04 & & $0.07^{* \star *}$ & 0.02 & & $0.10^{*}$ & & 0.00 & 0.06 \\
\hline \multirow{2}{*}{ Fixed Invest } & $\mathrm{B}_{\mathrm{k} 0}$ & & & & & 0.00 & & & & \\
\hline & $\theta_{\mathrm{k} 0}$ & & & & & -0.01 & & & & \\
\hline \multirow{2}{*}{ Gross Domestic Product } & $\mathrm{B}_{\mathrm{k} 0}$ & $0.01^{\star \star \star}$ & 0.00 & -0.02 & -0.01 & 0.01 & 0.02 & 0.01 & $-0.08^{\star \star *}$ & 0.00 \\
\hline & $\theta_{\mathrm{k} 0}$ & $0.02^{\star *}$ & 0.01 & 0.00 & -0.01 & -0.01 & 0.01 & -0.01 & $0.16^{* * *}$ & 0.01 \\
\hline \multirow{2}{*}{ Imports } & $\mathrm{B}_{\mathrm{k} 0}$ & -0.01 & & 0.00 & 0.01 & & 0.00 & & & -0.02 \\
\hline & $\theta_{\mathrm{k} 0}$ & 0.04 & & $-0.26^{* * *}$ & -0.04 & & 0.02 & & & 0.00 \\
\hline \multirow{2}{*}{ Interest rate } & $\mathrm{B}_{\mathrm{k} 0}$ & & & & & & & 0.00 & 0.00 & \\
\hline & $\theta_{\mathrm{k} 0}$ & & & & & & & $0.42^{* * *}$ & -0.03 & \\
\hline \multirow{2}{*}{ Industrial production } & $\mathrm{B}_{\mathrm{k} 0}$ & $0.01^{* *}$ & $-0.01^{*}$ & & 0.00 & $0.01^{* * *}$ & & & & 0.00 \\
\hline & $\theta_{\mathrm{k} 0}$ & 0.00 & $0.03^{* * *}$ & & -0.01 & 0.00 & & & & -0.01 \\
\hline \multirow{2}{*}{ Money Supply } & $\mathrm{B}_{\mathrm{k} 0}$ & 0.00 & & & & & -0.02 & $0.04^{\star * *}$ & & \\
\hline & $\theta_{\mathrm{k} 0}$ & 0.02 & & & & & 0.01 & 0.00 & & \\
\hline \multirow{2}{*}{ Producer Price } & $\mathrm{B}_{\mathrm{k} 0}$ & 0.00 & -0.01 & & & $0.00^{* * *}$ & -0.02 & 0.00 & & $0.16^{\star * *}$ \\
\hline & $\theta_{\mathrm{k} 0}$ & 0.01 & $0.02^{\star * *}$ & & & 0.01 & $0.04^{*}$ & 0.01 & & 0.02 \\
\hline \multirow{2}{*}{ Retail Sales } & $\mathrm{B}_{\mathrm{k} 0}$ & 0.01 & & & & 0.00 & 0.01 & $-0.08^{* * *}$ & & \\
\hline & $\theta_{\mathrm{k} 0}$ & $0.02^{* * *}$ & & & & -0.01 & $0.02^{*}$ & 0.05 & & \\
\hline \multirow{2}{*}{ Trade Balance } & $\mathrm{B}_{\mathrm{k} 0}$ & $0.01^{*}$ & 0.00 & 0.17 & & 0.01 & & $0.09^{* * *}$ & & -0.03 \\
\hline & $\theta_{\mathrm{k} 0}$ & $0.02^{* * *}$ & 0.01 & $0.19^{* * \star}$ & & -0.01 & & 0.00 & & 0.05 \\
\hline \multirow{2}{*}{ Initial Unemployment } & $\mathrm{B}_{\mathrm{k} 0}$ & 0.01 & & & 0.00 & $0.03^{* * *}$ & -0.01 & & & -0.01 \\
\hline & $\theta_{\mathrm{k} 0}$ & 0.00 & & & -0.01 & -0.01 & 0.00 & & & -0.02 \\
\hline \multirow{2}{*}{ Wholesales } & $\mathrm{B}_{\mathrm{k} 0}$ & & & & & 0.00 & 0.01 & & & \\
\hline & $\theta_{\mathrm{k} 0}$ & & & & & 0.01 & 0.00 & & & \\
\hline
\end{tabular}


Appendix 6. Regression Results with Expected Appreciation

\begin{tabular}{|c|c|c|c|c|c|c|c|c|c|}
\hline \multirow[b]{2}{*}{ Announcements } & \multicolumn{6}{|c|}{ Czech } & \multicolumn{3}{|l|}{ South } \\
\hline & \multicolumn{4}{|c|}{ Republic Hungary Indonesia Korea } & Mexico & Poland & Africa & \multicolumn{2}{|c|}{ Thailand Turkey } \\
\hline \multicolumn{10}{|c|}{ Impact of Contemporaneous News Surprises on FX Return } \\
\hline Business Inventories & -0.01 & -0.01 & 0.01 & -0.01 & 0.01 & -0.01 & $-0.04^{\star *}$ & 0.00 & -0.03 \\
\hline Budget Deficit & -0.01 & 0.05 & 0.01 & 0.02 & -0.01 & 0.00 & 0.04 & 0.02 & 0.00 \\
\hline Current Account & $0.10^{* *}$ & $0.10^{* *}$ & $-0.06^{* *}$ & 0.01 & -0.01 & $0.03^{*}$ & 0.03 & 0.03 & 0.03 \\
\hline Capital Utilization & -0.01 & -0.02 & -0.02 & $-0.03^{* *}$ & 0.01 & 0.01 & $0.08^{* *}$ & 0.00 & $0.06^{*}$ \\
\hline Consumer Confidence & $0.12^{* *}$ & $0.10^{* *}$ & 0.02 & 0.02 & 0.02 & $0.07^{* *}$ & $0.05^{\star *}$ & 0.00 & 0.10 \\
\hline Consumer Credit & -0.01 & -0.01 & -0.01 & -0.01 & -0.01 & 0.00 & -0.02 & -0.01 & 0.31 \\
\hline Construction Spending & $0.06^{* *}$ & 0.02 & 0.01 & 0.01 & 0.00 & $0.03^{* *}$ & -0.02 & $-0.05^{\star *}$ & -0.01 \\
\hline Consumer Price Index & -0.02 & 0.02 & $-0.04^{\star *}$ & 0.01 & $0.02^{*}$ & 0.01 & $0.05^{* *}$ & 0.00 & 0.05 \\
\hline Durable Goods Orders & $0.05^{\star *}$ & $0.07^{\star *}$ & $0.06^{* *}$ & $0.02^{*}$ & 0.01 & $0.06^{* *}$ & $0.06^{\star *}$ & -0.02 & 0.04 \\
\hline Factory Orders & $0.05^{\star *}$ & 0.02 & 0.00 & $-0.03^{*}$ & 0.01 & 0.00 & $0.03^{*}$ & 0.00 & 0.02 \\
\hline Gross Domestic Product & $0.09^{* *}$ & $0.09^{* *}$ & $0.06^{* *}$ & 0.02 & 0.01 & $0.07^{* *}$ & $0.09^{* *}$ & 0.00 & 0.00 \\
\hline Housing Starts & 0.02 & $0.03^{*}$ & -0.01 & $0.02^{*}$ & 0.01 & 0.01 & 0.02 & -0.01 & -0.03 \\
\hline Imports & 0.01 & 0.01 & $0.03^{*}$ & 0.02 & 0.00 & 0.00 & -0.02 & -0.01 & $-0.05^{*}$ \\
\hline Interest rate & 0.01 & 0.02 & 0.00 & 0.00 & 0.01 & 0.00 & 0.00 & 0.01 & 0.00 \\
\hline Industrial production & $0.07^{* *}$ & 0.04 & $0.03^{*}$ & 0.02 & 0.00 & 0.01 & -0.02 & 0.01 & -0.01 \\
\hline NAPM & $0.61^{*}$ & $0.83^{* *}$ & 0.40 & $0.72^{* *}$ & 0.24 & $0.78^{* *}$ & 0.55 & -0.23 & 0.52 \\
\hline Leading Indicators & -0.01 & 0.02 & 0.00 & -0.01 & 0.00 & 0.00 & -0.01 & 0.00 & 0.00 \\
\hline New Home Sales & -0.02 & $0.03^{*}$ & $0.04^{* *}$ & $0.03^{* *}$ & 0.01 & $0.03^{* *}$ & -0.01 & -0.01 & -0.04 \\
\hline Nonfarm Payroll & $0.19^{* *}$ & $0.21^{* *}$ & $0.07^{* *}$ & $0.08^{* *}$ & $0.11^{* *}$ & $0.23^{* *}$ & $0.26^{* *}$ & 0.01 & $0.14^{* *}$ \\
\hline Personal Spending & -0.01 & 0.01 & 0.02 & 0.00 & 0.01 & 0.00 & -0.01 & -0.01 & 0.00 \\
\hline Personal Income & 0.03 & 0.02 & 0.00 & 0.01 & 0.01 & 0.00 & $0.03^{*}$ & 0.00 & 0.00 \\
\hline Producer Price & -0.01 & -0.01 & $0.02^{*}$ & 0.01 & 0.01 & 0.01 & 0.03 & 0.00 & 0.02 \\
\hline Retail Sales & $0.09^{* *}$ & $0.07^{* *}$ & $0.04^{* *}$ & $0.04^{* *}$ & 0.02 & $0.08^{* *}$ & $0.03^{*}$ & 0.01 & -0.01 \\
\hline Trade Balance & $0.04^{* *}$ & $0.06^{\star *}$ & -0.01 & 0.01 & -0.01 & $0.11^{* *}$ & $0.09^{* *}$ & 0.01 & 0.03 \\
\hline Initial Unemployment & $-0.04^{* *}$ & $-0.03^{* *}$ & -0.01 & $-0.02^{* *}$ & $-0.02^{* *}$ & $-0.02^{* *}$ & $-0.02^{* *}$ & 0.01 & 0.01 \\
\hline Wholesales & 0.00 & 0.01 & 0.01 & 0.01 & -0.03 & 0.00 & 0.00 & 0.00 & -0.01 \\
\hline Budget Deficit & -0.05 & -0.34 & & & & 0.01 & & & \\
\hline Current Account & -0.02 & $-0.04^{*}$ & & & -0.07 & $-0.07^{* *}$ & $0.34^{* *}$ & 0.03 & 0.03 \\
\hline Current Account(US) & -0.01 & & & & & & & & \\
\hline Consumer Confidence & & & & & -0.01 & & & & 0.00 \\
\hline Consumer Price Index & $-0.03^{*}$ & $0.05^{* *}$ & & 0.01 & 0.01 & 0.01 & $-0.04^{*}$ & -0.01 & $1.74^{* *}$ \\
\hline Exports & $-0.41^{* *}$ & & -0.01 & 0.01 & & 0.00 & & 0.01 & -0.20 \\
\hline Fixed Invest & & & & & 0.00 & & & & \\
\hline Gross Domestic Product & 0.01 & -0.16 & -0.01 & -0.05 & 0.00 & $-0.06^{* *}$ & -0.08 & $-0.16^{* *}$ & -0.03 \\
\hline Imports & 0.08 & & 0.02 & 0.00 & & -0.01 & & & 0.00 \\
\hline Interest rate & & & & & & & $-0.36^{\star *}$ & -0.01 & \\
\hline Industrial production & -0.01 & -0.02 & & 0.00 & -0.02 & & & & -0.02 \\
\hline Money Supply & 0.00 & & & & & -0.01 & 0.00 & & \\
\hline Producer Price & -0.02 & $-0.02^{*}$ & & & 0.01 & 0.00 & 0.02 & & $0.14^{* *}$ \\
\hline Retail Sales & -0.02 & & & & -0.01 & -0.01 & 0.01 & & \\
\hline Trade Balance & $-0.09^{* *}$ & -0.01 & 2.03 & & -0.01 & & $-0.14^{* *}$ & & 0.06 \\
\hline Initial Unemployment & $0.35^{\star *}$ & & & -0.04 & -0.01 & 0.01 & & & 0.00 \\
\hline Wholesales & & & & & -0.02 & $-0.03^{* *}$ & & & \\
\hline
\end{tabular}




\begin{tabular}{|c|c|c|c|c|c|c|c|c|c|}
\hline \multicolumn{10}{|c|}{ Impact of Contemporaneous News Surprises Multiplied by Appreciation Expectation } \\
\hline Business Inventories & -0.05 & -0.05 & $-1.37^{*}$ & -0.29 & 0.00 & $-0.77^{*}$ & -0.28 & 1.05 & 0.00 \\
\hline Budget Deficit & -0.06 & $-0.31^{*}$ & -0.26 & $7.53^{* *}$ & -0.01 & -0.34 & 0.53 & -0.42 & 0.00 \\
\hline Capital Utilization & 0.01 & 0.11 & 0.04 & $-1.88^{*}$ & $0.04^{*}$ & -0.03 & $-1.20^{* *}$ & 0.22 & 0.00 \\
\hline Current Account & $-0.23^{*}$ & -0.26 & 1.41 & 0.09 & -0.02 & 0.38 & 0.04 & -1.53 & 0.00 \\
\hline Consumer Credit & -0.02 & -0.08 & 0.23 & -0.18 & -0.01 & -0.15 & 0.35 & 0.65 & -6.12 \\
\hline Consumer Confidence & $-0.11^{*}$ & -0.03 & 0.52 & $2.35^{\star *}$ & $0.05^{* *}$ & $-1.02^{\star *}$ & 0.15 & 0.65 & 0.00 \\
\hline Construction Spending & $-0.18^{*}$ & 0.01 & -0.06 & $-1.57^{*}$ & 0.00 & $0.96^{*}$ & $0.68^{*}$ & $2.34^{*}$ & 0.00 \\
\hline Consumer Price Index & $0.18^{* *}$ & 0.06 & $-2.67^{\star *}$ & 0.23 & 0.00 & -0.46 & 0.28 & 0.96 & 0.00 \\
\hline Durable Goods Orders & $0.22^{* *}$ & $0.19^{* *}$ & $1.08^{*}$ & 0.39 & $0.04^{* *}$ & 0.10 & 0.29 & -1.04 & $0.00^{*}$ \\
\hline Factory Orders & $-0.12^{* *}$ & 0.02 & 0.05 & $1.68^{*}$ & 0.02 & -0.03 & 0.34 & 0.14 & 0.00 \\
\hline Gross Domestic Product & $0.20^{* *}$ & $0.24^{* *}$ & $0.87^{*}$ & $0.81^{*}$ & 0.03 & 0.17 & -0.51 & -0.18 & 0.00 \\
\hline Housing Starts & 0.02 & -0.08 & -0.21 & -0.93 & 0.01 & 0.46 & -0.19 & -0.47 & $0.00^{*}$ \\
\hline Imports & 0.02 & -0.04 & $3.19^{* *}$ & 0.39 & $-0.04^{* *}$ & 0.05 & $-1.26^{* *}$ & -0.59 & 0.00 \\
\hline Interest rate & 0.00 & 0.12 & 0.00 & 0.00 & 0.00 & 0.00 & 0.00 & 0.00 & 0.00 \\
\hline Industrial production & $-0.21^{* *}$ & -0.09 & 0.29 & 1.62 & -0.01 & 0.24 & -0.20 & -0.21 & 0.00 \\
\hline NAPM & $2.55^{*}$ & 2.20 & 12.73 & 17.15 & 0.45 & 3.42 & -7.16 & 12.46 & $0.00^{*}$ \\
\hline Leading Indicators & 0.09 & 0.02 & 0.39 & 0.49 & 0.02 & 0.40 & 0.26 & 0.38 & 0.00 \\
\hline New Home Sales & $0.18^{* *}$ & -0.01 & $0.89^{*}$ & -0.75 & 0.01 & 0.00 & 0.34 & 0.28 & 0.00 \\
\hline Nonfarm Payroll & $0.73^{* *}$ & $0.65^{* *}$ & $1.36^{* *}$ & $1.07^{*}$ & $0.10^{* *}$ & $-0.52^{*}$ & -0.13 & $-1.80^{\star *}$ & $0.00^{* *}$ \\
\hline Personal Spending & 0.08 & 0.00 & 0.26 & 0.26 & 0.01 & 0.17 & 0.06 & -0.19 & 0.00 \\
\hline Personal Income & $-0.14^{*}$ & -0.10 & 0.50 & 0.93 & 0.01 & -0.43 & $1.57^{* *}$ & -0.14 & 0.00 \\
\hline Producer Price & $0.13^{* *}$ & $0.18^{* *}$ & 0.50 & 0.16 & 0.01 & 0.24 & 0.11 & 0.43 & $0.00^{*}$ \\
\hline Retail Sales & 0.06 & $0.13^{*}$ & $1.85^{\star *}$ & -0.29 & $0.12^{* *}$ & 0.30 & 0.39 & -0.49 & 0.00 \\
\hline Trade Balance & $0.38^{* *}$ & $0.31^{* *}$ & -0 & $1.12^{*}$ & -0.01 & $-0.84^{*}$ & $-1.00^{* *}$ & 0.06 & 0.00 \\
\hline Initial Unemployment & -0.01 & -0.04 & -0.09 & -0.16 & $-0.03^{* *}$ & $0.44^{* *}$ & 0.14 & 0.32 & 0.00 \\
\hline Wholesales & -0.01 & -0.06 & 0.34 & $-1.28^{*}$ & -0.03 & -0.28 & $0.76^{\star *}$ & 0.11 & 0.00 \\
\hline Budget Deficit & 0.09 & 1.52 & & & & 0.53 & & & \\
\hline Current Account & -0.03 & $-0.51^{* *}$ & & & -0.07 & $1.32^{* *}$ & $-8.49^{* *}$ & $11.61^{\star *}$ & 0.00 \\
\hline Current Account(US) & -0.09 & & & & & & & & \\
\hline Consumer Confidence & & & & & -0.01 & & & & 0.00 \\
\hline Consumer Price Index & $0.15^{*}$ & -0.06 & & 0.24 & -0.01 & -0.40 & $1.00^{* *}$ & -0.26 & $0.00^{* *}$ \\
\hline Exports & $1.42^{* *}$ & & -0.34 & -0.49 & & $-1.07^{*}$ & & -1.64 & 8.92 \\
\hline Fixed Invest & & & & & -0.02 & & & & \\
\hline Gross Domestic Product & $-0.38^{* *}$ & 0.48 & -0.02 & -1.84 & 0.04 & $2.02^{*}$ & 0.30 & -5.16 & 0.00 \\
\hline Imports & 1.29 & & 0.77 & 0.29 & & $1.99^{* *}$ & & & $9.11^{* *}$ \\
\hline Interest rate & & & & & & & $-7.96^{* *}$ & 0.00 & \\
\hline Industrial production & 0.00 & -0.08 & & 0.11 & -0.02 & & & & 0.00 \\
\hline Money Supply & -0.10 & & & & & 0.10 & $-2.15^{* *}$ & & \\
\hline Producer Price & -0.06 & 0.20 & & & 0.00 & -0.39 & $-0.83^{*}$ & & $-2.74^{\star *}$ \\
\hline Retail Sales & 0.05 & & & & 0.02 & -0.21 & -0.21 & & \\
\hline Trade Balance & 0.08 & 0.05 & 51.91 & & 0.01 & & -0.28 & & 0.00 \\
\hline Initial Unemployment & $-1.87^{\star *}$ & & & -2.18 & 0.02 & 0.22 & & & 0.00 \\
\hline Wholesales & & & & & 0.00 & -0.10 & & & \\
\hline
\end{tabular}

